\title{
COHOMOLOGY AND COQUASI-BIALGEBRAS IN THE CATEGORY OF YETTER-DRINFELD MODULES
}

\author{
IVÁN ANGIONO, ALESSANDRO ARDIZZONI, AND CLAUDIA MENINI
}

\begin{abstract}
We prove that a finite-dimensional Hopf algebra with the dual Chevalley Property over a field of characteristic zero is quasi-isomorphic to a Radford-Majid bosonization whenever the third Hochschild cohomology group in the category of Yetter-Drinfeld modules of its diagram with coefficients in the base field vanishes. Moreover we show that this vanishing occurs in meaningful examples where the diagram is a Nichols algebra.
\end{abstract}

\section{Contents}

Introduction

Preliminaries

1. Yetter-Drinfeld

2. Gauge deformation

3. (Co)semisimple case

4. Invariants

5. Dual Chevalley

6. Examples

6.1. Braidings of Cartan type

6.2. Braidings of non-diagonal type

References

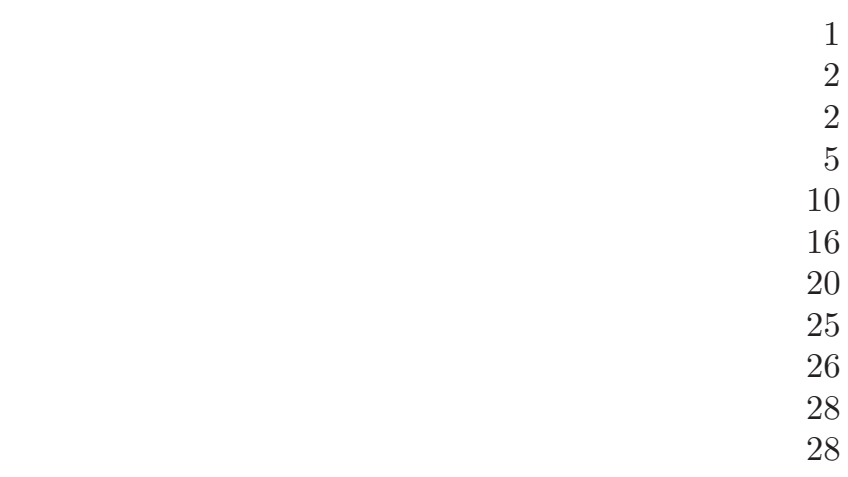

\section{INTRODUCTION}

Let $A$ be a finite-dimensional Hopf algebra over a field $\mathbb{k}$ of characteristic zero such that the coradical $H$ of $A$ is a sub-Hopf algebra (i.e. $A$ has the dual Chevalley Property). Denote by $\mathcal{D}(A)$ the diagram of $A$. The main aim of this paper (see Theorem 5.6) is to prove that, if the third Hochschild cohomology group in ${ }_{H}^{H} \mathcal{Y} \mathcal{D}$ of the algebra $\mathcal{D}(A)$ with coefficients in $\mathbb{k}$ vanishes, in symbols $\mathrm{H}_{\mathcal{Y} \mathcal{D}}^{3}(\mathcal{D}(A), \mathbb{k})=0$, then $A$ is quasi-isomorphic to the Radford-Majid bosonization $E \# H$ of some connected bialgebra $E$ in ${ }_{H}^{H} \mathcal{Y D}$ with gr $(E) \cong \mathcal{D}(A)$ as bialgebras in ${ }_{H}^{H} \mathcal{Y} \mathcal{D}$.

The paper is organized as follows. Let $H$ be a Hopf algebra over a field $\mathbb{k}$. In Section 1 i we investigate the properties of coalgebras with multiplication and unit in the category ${ }_{H}^{H} \mathcal{Y} \mathcal{D}$ (in particular of coquasi-bialgebras) and their associated graded coalgebra. The main result of this section, Theorem 1.5, establishes that the associated graded coalgebra $\operatorname{gr} Q$ of a connected coquasibialgebra in ${ }_{H}^{H} \mathcal{Y D}$ is a connected bialgebra in ${ }_{H}^{H} \mathcal{Y D}$.

2010 Mathematics Subject Classification. Primary 16W30; Secondary 16E40.

Key words and phrases. Hopf Algebras, Coquasi-bialgebras, Bosonizations, Cocycle Deformations, Hochschild Cohomology.

This paper was started during the visit, supported by INdAM, of the first author to the University of Ferrara in November 2012. It was written while both the second and the third authors were members of GNSAGA. The first author's work was partially supported by CONICET, FONCyT-ANPCyT, Secyt (UNC). The second author was partially supported by the research grant "Progetti di Eccellenza 2011/2012" from the "Fondazione Cassa di Risparmio di Padova e Rovigo". 
In Section 2 we study the deformation of coquasi-bialgebras in ${ }_{H}^{H} \mathcal{Y D}$ by means of gauge transformations. In Proposition 2.5 we investigate its behaviour with respect to bosonization while in Proposition 2.6 with respect to the associated graded coalgebra.

In Section 3 we consider the associated graded coalgebra in case the Hopf algebra $H$ is semisimple and cosemisimple (e.g. $H$ is finite-dimensional cosemisimple over a field of characteristic zero). In particular, in Theorem 3.2, we prove that a f.d. connected coquasi-bialgebra $Q$ in ${ }_{H}^{H} \mathcal{Y} \mathcal{D}$ is gauge equivalent to a connected bialgebra in ${ }_{H}^{H} \mathcal{Y} \mathcal{D}$ whenever $\mathrm{H}_{\mathcal{Y} \mathcal{D}}^{3}(\operatorname{gr} Q, \mathbb{k})=0$. This result is inspired by EG, Proposition 2.3].

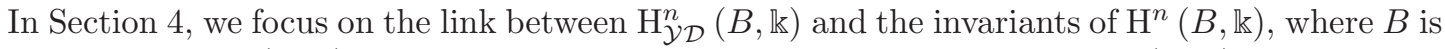
a bialgebra in $\mathrm{H}_{\mathcal{Y} \mathcal{D}}^{n}(B, \mathbb{k})$. In particular, in Proposition 4.7 we show that $\mathrm{H}_{\mathcal{Y} \mathcal{D}}^{n}(B, \mathbb{k})$ is isomorphic to $\mathrm{H}^{n}(B, \mathbb{k})^{D(H)}$, which is a subspace of $\mathrm{H}^{n}(B, \mathbb{k})^{H} \cong \mathrm{H}^{n}(B \# H, \mathbb{k})$, see Corollary 4.3. 5.6 .

Section 5 is devoted to the proof of the main result of the paper, the aforementioned Theorem

In Section 6 we provide examples where $\mathrm{H}_{\mathcal{Y} \mathcal{D}}^{n}(B, \mathbb{k})=0$ in case $B$ is the Nichols algebra $\mathcal{B}(V)$ of a Yetter-Drinfeld module $V$. In particular we show that that $\mathrm{H}_{\mathcal{Y} \mathcal{D}}^{3}(\mathcal{B}(V), \mathbb{k})$ can be zero although $\mathrm{H}^{3}(\mathcal{B}(V) \# H, \mathbb{k})$ is non-trivial.

\section{PRELIMINARIES}

Given a category $\mathcal{C}$ and objects $M, N \in \mathcal{C}$, the notation $\mathcal{C}(M, N)$ stands for the set of morphisms in $\mathcal{C}$. This notation will be mainly applied to the case $\mathcal{C}$ is the category of vector space $\mathbf{V e c}_{\mathbb{k}}$ over a field $\mathbb{k}$ or $\mathcal{C}$ is the category of Yetter-Drinfeld modules ${ }_{H}^{H} \mathcal{Y} \mathcal{D}$ over a Hopf algebra $H$. The set of natural numbers including 0 is denoted by $\mathbb{N}_{0}$ while $\mathbb{N}$ denotes the same set without 0 .

\section{YetTER-DRINFELD}

Definition 1.1. Let $C$ be a coalgebra. Denote by $C_{n}$ the $n$-th term of the coradical filtration of $C$ and set $C_{-1}:=0$. For every $x \in C$, we set

$$
|x|:=\min \left\{i \in \mathbb{N}_{0}: x \in C_{i}\right\} \quad \text { and } \quad \bar{x}:=x+C_{|x|-1} .
$$

Note that, for $x=0$, we have $|x|=0$. One can define the associated graded coalgebra

$$
\operatorname{gr} C:=\oplus_{i \in \mathbb{N}_{0}} \frac{C_{i}}{C_{i-1}}
$$

with structure given, for every $x \in C$, by

$$
\begin{aligned}
\Delta_{\operatorname{gr} C}(\bar{x}) & =\sum_{0 \leq i \leq|x|}\left(x_{1}+C_{i-1}\right) \otimes\left(x_{2}+C_{|x|-i-1}\right), \\
\varepsilon_{\operatorname{gr} C}(\bar{x}) & =\delta_{|x|, 0} \varepsilon_{C}(x) .
\end{aligned}
$$

1.2. For every $i \in \mathbb{N}_{0}$, take a basis $\left\{\overline{x^{i, j}} \mid j \in B_{i}\right\}$ of the $\mathbb{k}$-module $C_{i} / C_{i-1}$ with $\overline{x^{i, j}} \neq \overline{x^{i, l}}$ for $j \neq l$ and

$$
\left|x^{i, j}\right|=i \text {. }
$$

Then $\left\{x^{i, j} \mid 0 \leq i \leq n, j \in B_{i}\right\}$ is a basis of $C_{n}$ and $\left\{x^{i, j} \mid i \in \mathbb{N}_{0}, j \in B_{i}\right\}$ is a basis of $C$. Assume that $C$ has a distinguished grouplike element $1=1_{C} \neq 0$ and take $i>0$. If $\varepsilon\left(x^{i, j}\right) \neq 0$ then we have that

$$
\overline{x^{i, j}-\varepsilon\left(x^{i, j}\right) 1}=\overline{x^{i, j}}
$$

so that we can take $x^{i, j}-\varepsilon\left(x^{i, j}\right) 1$ in place of $x^{i, j}$. In other words we can assume

$$
\varepsilon\left(x^{i, j}\right)=0 \text {, for every } i>0, j \in B_{i} .
$$

It is well-known there is a $\mathbb{k}$-linear isomorphism $\varphi: C \rightarrow \operatorname{gr} C$ defined on the basis by $\varphi\left(x^{i, j}\right):=\overline{x^{i, j}}$.

We compute

$$
\varepsilon_{\mathrm{gr} C} \varphi\left(x^{i, j}\right)=\varepsilon_{\mathrm{gr} C}\left(\overline{x^{i, j}}\right) \underline{\text { 国 }} \delta_{i, 0} \varepsilon\left(x^{0, j}\right) \stackrel{(\beta)}{\underline{B}} \varepsilon\left(x^{i, j}\right)
$$


Hence we obtain

$$
\varepsilon_{\mathrm{gr} C} \circ \varphi=\varepsilon .
$$

Let $H$ be a Hopf algebra. A coalgebra with multiplication and unit in ${ }_{H}^{H} \mathcal{Y D}$ is a datum $(Q, m, u, \Delta, \varepsilon)$ where $(Q, \Delta, \varepsilon)$ is a coalgebra in ${ }_{H}^{H} \mathcal{Y} \mathcal{D}, m: Q \otimes Q \rightarrow Q$ is a coalgebra morphism in ${ }_{H}^{H} \mathcal{Y D}$ called multiplication (which may fail to be associative) and $u: \mathbb{k} \rightarrow Q$ is a coalgebra morphism in ${ }_{H}^{H} \mathcal{Y D}$ called unit. In this case we set $1_{Q}:=u\left(1_{\mathbb{k}}\right)$.

Note that, for every $h \in H, k \in \mathbb{k}$, we have

(6) $\left(1_{Q}\right)_{-1} \otimes\left(1_{Q}\right)_{0}=\left(u\left(1_{\mathbb{k}}\right)\right)_{-1} \otimes\left(u\left(1_{\mathbb{k}}\right)\right)_{0}=\left(1_{\mathbb{k}}\right)_{-1} \otimes u\left(\left(1_{\mathbb{k}}\right)_{0}\right)=1_{H} \otimes u\left(1_{\mathbb{k}}\right)=1_{H} \otimes 1_{Q}$.

Proposition 1.3. Let $H$ be a Hopf algebra and let $(Q, m, u, \Delta, \varepsilon)$ be a coalgebra with multiplication and unit in ${ }_{H}^{H} \mathcal{Y D}$. If $Q_{0}$ is a subcoalgebra of $Q$ in ${ }_{H}^{H} \mathcal{Y D}$ such that $Q_{0} \cdot Q_{0} \subseteq Q_{0}$, then $Q_{n}$ is a subcoalgebra of $Q$ in ${ }_{H}^{H} \mathcal{Y D}$ for every $n \in \mathbb{N}_{0}$. Moreover $Q_{a} \cdot Q_{b} \subseteq Q_{a+b}$ for every $a, b \in \mathbb{N}_{0}$ and the graded coalgebra $\operatorname{gr} Q$, associated with the coradical filtration of $Q$, is a coalgebra with multiplication and unit in ${ }_{H}^{H} \mathcal{Y D}$ with respect to the usual coalgebra structure and with multiplication and unit defined by

$$
\begin{aligned}
m_{\mathrm{gr} Q}\left(\left(x+Q_{a-1}\right) \otimes\left(y+Q_{b-1}\right)\right) & :=x y+Q_{a+b-1}, \\
u_{\mathrm{gr} Q}(k) & :=k 1_{Q}+Q_{-1}
\end{aligned}
$$

Proof. The coalgebra structure of $Q$ induces a coalgebra structure on $\operatorname{gr} Q$. Since $Q_{0}$ is a subcoalgebra of $Q$ in ${ }_{H}^{H} \mathcal{Y D}$ and, for $n \geq 1$, one has $Q_{n}=Q_{n-1} \wedge_{Q} Q_{0}$, then inductively one proves that $Q_{n}$ is a subcoalgebra of $Q$ in ${ }_{H}^{H} \mathcal{Y D}$. As a consequence one gets that $\operatorname{gr} Q$ is a coalgebra in ${ }_{H}^{H} \mathcal{Y} \mathcal{D}$ (this construction can be performed in the setting of monoidal categories under suitable assumptions, see e.g. AM, Theorem 2.10]). Let us prove that $\operatorname{gr} Q$ inherits also a multiplication and unit. Let us check that $Q_{a} \cdot Q_{b} \subseteq Q_{a+b}$ for every $a, b \in \mathbb{N}_{0}$. We proceed by induction on $n=a+b$. If $n=0$ there is nothing to prove. Let $n \geq 1$ and assume that $Q_{i} \cdot Q_{j} \subseteq Q_{i+j}$ for every $i, j \in \mathbb{N}_{0}$ such that $0 \leq i+j \leq n-1$. Let $a, b \in \mathbb{N}_{0}$ be such that $n=a+b$. Since $\Delta\left(Q_{a}\right) \subseteq \sum_{i=0}^{a} Q_{i} \otimes Q_{a-i}$ and $c_{Q, Q}\left(Q_{u} \otimes Q_{v}\right) \subseteq Q_{v} \otimes Q_{u}$, where $c_{Q, Q}$ denotes the braiding in ${ }_{H}^{H} \mathcal{Y} \mathcal{D}$, using the compatibility condition between $\Delta$ and $m$, one easily gets that $\Delta\left(Q_{a} \cdot Q_{b}\right) \subseteq Q_{a+b-1} \otimes Q+Q \otimes Q_{0}$.

Therefore $Q_{a} \cdot Q_{b} \subseteq Q_{a+b}$. This property implies we have a well-defined map in ${ }_{H}^{H} \mathcal{Y D}$

$$
m_{\mathrm{grQ}}^{a, b}: \frac{Q_{a}}{Q_{a-1}} \otimes \frac{Q_{b}}{Q_{b-1}} \rightarrow \frac{Q_{a+b}}{Q_{a+b-1}}
$$

defined, for $x \in Q_{a}$ and $y \in Q_{b}$, by (7). This can be seen as the graded component of a morphism in ${ }_{H}^{H} \mathcal{Y D}$ that we denote by $m_{\operatorname{gr} Q}: \operatorname{gr} Q \otimes \operatorname{gr} Q \rightarrow \operatorname{gr} Q$. Let us check that $m_{\mathrm{gr} Q}$ is a coalgebra morphism in ${ }_{H}^{H} \mathcal{Y D}$. Consider a basis of $Q$ with terms of the form $x^{i, j}$ as in 1.2. Hence we can write the comultiplication in the form

$$
\Delta\left(x^{a, u}\right)=\sum_{s+t \leq a} \sum_{l, m} \eta_{s, t, l, m}^{a, u} x^{s, l} \otimes x^{t, m} .
$$

Now, using (1), one gets that

$$
\Delta_{\mathrm{gr} Q}\left(\overline{x^{a, u}}\right)=\sum_{0 \leq i \leq a} \sum_{l, m} \eta_{i, a-i, l, m}^{a, u} \overline{x^{i, l}} \otimes \overline{x^{a-i, m}} .
$$

Using that $\Delta_{\operatorname{gr} Q \otimes \operatorname{gr} Q}=\left(\operatorname{gr} Q \otimes c_{\operatorname{gr} Q, \operatorname{gr} Q} \otimes \operatorname{gr} Q\right)\left(\Delta_{\operatorname{gr} Q} \otimes \Delta_{\operatorname{gr} Q}\right)$ and (8), it is straightforward to check that $\left(m_{\mathrm{gr} Q} \otimes m_{\mathrm{gr} Q}\right) \Delta_{\mathrm{gr} Q \otimes \operatorname{gr} Q}\left(\overline{x^{a, u}} \otimes \overline{x^{b, v}}\right)=\Delta_{\mathrm{gr} Q} m_{\mathrm{gr} Q}\left(\overline{x^{a, u}} \otimes \overline{x^{b, v}}\right)$.

Moreover, since $\varepsilon_{\operatorname{gr} Q \otimes \operatorname{gr} Q}=\varepsilon_{\operatorname{gr} Q} \otimes \varepsilon_{\operatorname{gr} Q}$, we get that $\varepsilon_{\operatorname{gr} Q} m_{\operatorname{gr} Q}\left(\overline{x^{a, u}} \otimes \overline{x^{b, v}}\right)=\varepsilon_{\operatorname{gr} Q \otimes \operatorname{gr} Q}\left(\overline{x^{a, u}} \otimes \overline{x^{b, v}}\right)$.

This proves that $m_{\mathrm{gr} Q}$ is a coalgebra morphism in ${ }_{H}^{H} \mathcal{Y} \mathcal{D}$.

The fact that $u_{\mathrm{gr} Q}: \mathbb{k} \rightarrow \operatorname{gr} Q$, defined by $u_{\mathrm{gr} Q}(k):=k 1_{Q}+Q_{-1}$ is a coalgebra morphism in ${ }_{H}^{H} \mathcal{Y D}$ easily follows by means of (5) and (5). 
Definition 1.4 ([ABM, Definition 5.2]). Let $H$ be a Hopf algebra. Recall that a coquasi-bialgebra $(Q, m, u, \Delta, \varepsilon, \alpha)$ in the pre-braided monoidal category ${ }_{H}^{H} \mathcal{Y} \mathcal{D}$ is a coalgebra $(Q, \Delta, \varepsilon)$ in ${ }_{H}^{H} \mathcal{Y} \mathcal{D}$ together with coalgebra homomorphisms $m: Q \otimes Q \rightarrow Q$ and $u: \mathbb{k} \rightarrow Q$ in ${ }_{H}^{H} \mathcal{Y} \mathcal{D}$ and a convolution invertible element $\alpha \in{ }_{H}^{H} \mathcal{Y} \mathcal{D}\left(Q^{\otimes 3}, \mathbb{k}\right)$ (braided reassociator) such that

$$
\begin{aligned}
& \alpha(Q \otimes Q \otimes m) * \alpha(m \otimes Q \otimes Q)=(\varepsilon \otimes \alpha) * \alpha(Q \otimes m \otimes Q) *(\alpha \otimes \varepsilon), \\
& \alpha(Q \otimes u \otimes Q)=\alpha(u \otimes Q \otimes Q)=\alpha(Q \otimes Q \otimes u)=\varepsilon_{Q \otimes Q}, \\
& m(Q \otimes m) * \alpha=\alpha * m(m \otimes Q), \\
& m(u \otimes Q)=\operatorname{Id}_{Q}=m(Q \otimes u) .
\end{aligned}
$$

Here $*$ denotes the convolution product, where $Q^{\otimes 3}$ is the tensor product of coalgebras in ${ }_{H}^{H} \mathcal{Y D}$ whence it depends on the braiding of this category. Note that in (10) any of the three equalities such as $\alpha(u \otimes Q \otimes Q)=\varepsilon_{Q \otimes Q}$ implies that $\alpha$ is unital.

THEOREM 1.5. Let $H$ be a Hopf algebra and let $(Q, m, u, \Delta, \varepsilon, \omega)$ be a connected coquasi-bialgebra in ${ }_{H}^{H} \mathcal{Y} \mathcal{D}$. Then $\operatorname{gr} Q$ is a connected bialgebra in ${ }_{H}^{H} \mathcal{Y} \mathcal{D}$.

Proof. By Proposition 1.3, we know that $\operatorname{gr} Q$ is a coalgebra with multiplication and unit in ${ }_{H}^{H} \mathcal{Y} \mathcal{D}$. We have to check that the multiplication is associative and unitary.

Given two coalgebras $D, E$ in ${ }_{H}^{H} \mathcal{Y} \mathcal{D}$ endowed with coalgebras filtration $\left(D_{(n)}\right)_{n \in \mathbb{N}_{0}}$ and $\left(E_{(n)}\right)_{n \in \mathbb{N}_{0}}$ in ${ }_{H}^{H} \mathcal{Y D}$ such that $D_{(0)}$ and $E_{(0)}$ are one-dimensional, let us check that $C_{(n)}:=\sum_{0 \leq i \leq n} D_{(i)} \otimes E_{(n-i)}$ gives a coalgebra filtration on $C:=D \otimes E$ in ${ }_{H}^{H} \mathcal{Y} \mathcal{D}$. First note that the coalgebra structure of $C$ depends on the braiding. Thus, we have

$$
\begin{aligned}
\Delta_{C}\left(C_{(n)}\right) & =\left(D \otimes c_{D, E} \otimes E\right)\left(\Delta_{D} \otimes \Delta_{E}\right)\left(\sum_{i=0}^{n} D_{(i)} \otimes E_{(n-i)}\right) \\
& \subseteq\left(D \otimes c_{D, E} \otimes E\right)\left(\sum_{i=0}^{n} \sum_{a=0}^{i} \sum_{b=0}^{n-i} D_{(a)} \otimes D_{(i-a)} \otimes E_{(b)} \otimes E_{(n-i-b)}\right) \\
& \subseteq \sum_{i=0}^{n} \sum_{a=0}^{i} \sum_{b=0}^{n-i} D_{(a)} \otimes c_{D, E}\left(D_{(i-a)} \otimes E_{(b)}\right) \otimes E_{(n-i-b)} \\
& \subseteq \sum_{i=0}^{n} \sum_{a=0}^{i} \sum_{b=0}^{n-i} D_{(a)} \otimes c_{D_{(i-a)}, E_{(b)}}\left(D_{(i-a)} \otimes E_{(b)}\right) \otimes E_{(n-i-b)} \\
& \subseteq \sum_{i=0}^{n} \sum_{a=0}^{i} \sum_{b=0}^{n-i} D_{(a)} \otimes E_{(b)} \otimes D_{(i-a)} \otimes E_{(n-i-b)} \\
& \subseteq \sum_{i=0}^{n} \sum_{w=0}^{n} \sum_{\substack{0 \leq a \leq i, 0 \leq b \leq n-i \\
a+b=w}} D_{(a)} \otimes E_{(b)} \otimes D_{(i-a)} \otimes E_{(n-i-b)} \\
& \subseteq \sum_{w=0}^{n} C_{(w)} \otimes C_{(n-w)} .
\end{aligned}
$$

Moreover, by $\mathrm{Sw}$, Proposition 11.1.1], we have that the coradical of $C$ is contained in $D_{(0)} \otimes E_{(0)}$ and hence it is one-dimensional.

This argument can be used to produce a coalgebra filtration on $C:=Q \otimes Q \otimes Q$ using as a filtration on $Q$ the coradical filtration. Let $n>0$ and let $w \in C_{(n)}=\sum_{i+j+k \leq n} Q_{i} \otimes Q_{j} \otimes Q_{k}$. By [AMS1, Lemma 3.69], we have that

$$
\Delta_{C}(w)-w \otimes\left(1_{Q}\right)^{\otimes 3}-\left(1_{Q}\right)^{\otimes 3} \otimes w \in C_{(n-1)} \otimes C_{(n-1)} .
$$

Thus we get

$$
w_{1} \otimes w_{2} \otimes w_{3}-\Delta_{C}(w) \otimes\left(1_{Q}\right)^{\otimes 3}-\Delta_{C}\left(\left(1_{Q}\right)^{\otimes 3}\right) \otimes w \in \Delta_{C}\left(C_{(n-1)}\right) \otimes C_{(n-1)}
$$

and hence, tensoring the first relation by $\left(1_{Q}\right)^{\otimes 3}$ on the right and adding it to the second one, we get

$w_{1} \otimes w_{2} \otimes w_{3}-w \otimes\left(1_{Q}\right)^{\otimes 3} \otimes\left(1_{Q}\right)^{\otimes 3}-\left(1_{Q}\right)^{\otimes 3} \otimes w \otimes\left(1_{Q}\right)^{\otimes 3}-\left(1_{Q}\right)^{\otimes 6} \otimes w \in C_{(n-1)} \otimes C_{(n-1)} \otimes C_{(n-1)}$.

For shortness, we set $\nu_{n}(z):=m(Q \otimes m)(z)+Q_{n-1}$ for every $z \in C$. Thus, by applying to the last displayed relation $C_{(n-1)} \otimes m(Q \otimes m) \otimes C_{(n-1)}$ and factoring out the middle term by $Q_{n-1}$, 
we get

$$
\begin{aligned}
& {\left[\begin{array}{c}
w_{1} \otimes \nu_{n}\left(w_{2}\right) \otimes w_{3}-w \otimes \nu_{n}\left(\left(1_{Q}\right)^{\otimes 3}\right) \otimes\left(1_{Q}\right)^{\otimes 3}+ \\
-\left(1_{Q}\right)^{\otimes 3} \otimes \nu_{n}(w) \otimes\left(1_{Q}\right)^{\otimes 3}-\left(1_{Q}\right)^{\otimes 3} \otimes \nu_{n}\left(\left(1_{Q}\right)^{\otimes 3}\right) \otimes w
\end{array}\right] } \\
\in \quad & C_{(n-1)} \otimes\left(\frac{\nu_{n}\left(C_{(n-1)}\right)}{Q_{n-1}}\right) \otimes C_{(n-1)} \subseteq C_{(n-1)} \otimes \frac{Q_{n-1}}{Q_{n-1}} \otimes C_{(n-1)}=0 .
\end{aligned}
$$

Thus we can express the first term with respect to the remaining ones as follows

$$
\begin{aligned}
& w_{1} \otimes \nu_{n}\left(w_{2}\right) \otimes w_{3} \\
= & w \otimes \nu_{n}\left(\left(1_{Q}\right)^{\otimes 3}\right) \otimes\left(1_{Q}\right)^{\otimes 3}+\left(1_{Q}\right)^{\otimes 3} \otimes \nu_{n}(w) \otimes\left(1_{Q}\right)^{\otimes 3}+\left(1_{Q}\right)^{\otimes 3} \otimes \nu_{n}\left(\left(1_{Q}\right)^{\otimes 3}\right) \otimes w \\
= & w \otimes\left(1_{Q}+Q_{n-1}\right) \otimes\left(1_{Q}\right)^{\otimes 3}+\left(1_{Q}\right)^{\otimes 3} \otimes \nu_{n}(w) \otimes\left(1_{Q}\right)^{\otimes 3}+\left(1_{Q}\right)^{\otimes 3} \otimes\left(1_{Q}+Q_{n-1}\right) \otimes w \\
\stackrel{n \geq 0}{=} & \left(1_{Q}\right)^{\otimes 3} \otimes \nu_{n}(w) \otimes\left(1_{Q}\right)^{\otimes 3} .
\end{aligned}
$$

We have so proved that for $n>0$ and $w \in C_{(n)}$

$$
w_{1} \otimes \nu_{n}\left(w_{2}\right) \otimes w_{3}=\left(1_{Q}\right)^{\otimes 3} \otimes \nu_{n}(w) \otimes\left(1_{Q}\right)^{\otimes 3}
$$

The same equation trivially holds also in the case $n=0$ as $C_{(n)}$ is one-dimensional.

Let $x, y, z \in Q$. Then $x \otimes y \otimes z \in C_{(|x|+|y|+|z|)}$ so that

$$
\begin{aligned}
(\bar{x} \cdot \bar{y}) \cdot \bar{z} & =\left(\left(x+Q_{|x|-1}\right) \cdot\left(y+Q_{|y|-1}\right)\right) \cdot\left(z+Q_{|z|-1}\right) \\
& =\left((x y)+Q_{|x|+|y|-1}\right) \cdot\left(z+Q_{|z|-1}\right) \\
& =(x y) z+Q_{|x|+|y|+|z|-1} \\
& =\omega^{-1}\left((x \otimes y \otimes z)_{1}\right) \nu_{|x|+|y|+|z|}\left((x \otimes y \otimes z)_{2}\right) \omega\left((x \otimes y \otimes z)_{3}\right) \\
& \text { (13) } \omega^{-1}\left(1_{Q} \otimes 1_{Q} \otimes 1_{Q}\right) \nu_{|x|+|y|+|z|}(x \otimes y \otimes z) \omega\left(1_{Q} \otimes 1_{Q} \otimes 1_{Q}\right) \\
& =\nu_{|x|+|y|+|z|}(x \otimes y \otimes z) \\
& =x(y z)+Q_{|x|+|y|+|z|-1}=\bar{x} \cdot(\bar{y} \cdot \bar{z}) .
\end{aligned}
$$

Therefore the multiplication is associative. It is also unitary as

$$
\bar{x} \cdot \overline{1_{Q}}=\left(x+Q_{|x|-1}\right) \cdot\left(1_{Q}+Q_{-1}\right)=x \cdot 1_{Q}+Q_{|x|-1}=x+Q_{|x|-1}=\bar{x}
$$

and similarly $\overline{1_{Q}} \cdot \bar{x}=\bar{x}$ for every $x \in Q$.

\section{Gauge Deformation}

Definition 2.1. Let $H$ be a Hopf algebra and let $(Q, m, u, \Delta, \varepsilon, \omega)$ be a coquasi-bialgebra in ${ }_{H}^{H} \mathcal{Y} \mathcal{D}$. A gauge transformation for $Q$ is a morphism $\gamma: Q \otimes Q \rightarrow \mathbb{k}$ in ${ }_{H}^{H} \mathcal{Y} \mathcal{D}$ which is convolution invertible in ${ }_{H}^{H} \mathcal{Y D}$ and which is also unitary on both entries.

REMARK 2.2. For $\gamma$ as above, let us check that $\gamma^{-1}$ is unitary whence a gauge transformation too.

First note that for all $x \in Q$, by means of (6) and (5), one gets

$$
\begin{aligned}
& \left(1_{Q} \otimes x\right)_{1} \otimes\left(1_{Q} \otimes x\right)_{2}=1_{Q} \otimes x_{1} \otimes 1_{Q} \otimes x_{2} \\
& \left(x \otimes 1_{Q}\right)_{1} \otimes\left(x \otimes 1_{Q}\right)_{2}=x_{1} \otimes 1_{Q} \otimes x_{2} \otimes 1_{Q}
\end{aligned}
$$

Thus

$$
\gamma^{-1}\left(1_{Q} \otimes x\right)=\gamma^{-1}\left(1_{Q} \otimes x_{1}\right) \varepsilon\left(x_{2}\right)=\gamma^{-1}\left(1_{Q} \otimes x_{1}\right) \gamma\left(1_{Q} \otimes x_{2}\right)=\left(\gamma^{-1} * \gamma\right)\left(1_{Q} \otimes x\right)=\varepsilon(x)
$$

and similarly $\gamma^{-1}\left(x \otimes 1_{Q}\right)=\varepsilon(x)$.

LEMma 2.3. Let $H$ be a Hopf algebra and let $C$ be a coalgebra in ${ }_{H}^{H} \mathcal{Y D}$. Given a map $\gamma \in$ ${ }_{H}^{H} \mathcal{Y D}(C, \mathbb{k})$, we have that $\gamma$ is convolution invertible in ${ }_{H}^{H} \mathcal{Y} \mathcal{D}(C, \mathbb{k})$ if and only if it is convolution invertible in $\mathbf{V e c}_{\mathbb{k}}(C, \mathbb{k})$. Moreover the inverse is the same. 
Proof. Assume there is a $\mathbb{k}$-linear map $\gamma^{-1}: C \rightarrow \mathbb{k}$ which is a convolution inverse of $\gamma$ in $\operatorname{Vec}_{\mathbb{k}}(C, \mathbb{k})$. By ABM1, Remark 2.4(ii)], $\gamma^{-1}$ is left $H$-linear. Let us check that $\gamma^{-1}$ is left $H$-colinear:

$$
\begin{aligned}
c_{-1} \otimes \gamma^{-1}\left(c_{0}\right) & =\left(c_{1}\right)_{-1} 1_{H} \otimes \gamma^{-1}\left(\left(c_{1}\right)_{0}\right) \gamma\left(c_{2}\right) \gamma^{-1}\left(c_{3}\right) \\
& =\left(c_{1}\right)_{-1}\left(c_{2}\right)_{-1} \otimes \gamma^{-1}\left(\left(c_{1}\right)_{0}\right) \gamma\left(\left(c_{2}\right)_{0}\right) \gamma^{-1}\left(c_{3}\right) \\
& \stackrel{(*)}{=}\left(c_{1}\right)_{-1} \otimes \gamma^{-1}\left(\left(\left(c_{1}\right)_{0}\right)_{1}\right) \gamma\left(\left(\left(c_{1}\right)_{0}\right)_{2}\right) \gamma^{-1}\left(c_{2}\right) \\
& =\left(c_{1}\right)_{-1} \otimes\left(\gamma^{-1} * \gamma\right)\left(\left(c_{1}\right)_{0}\right) \gamma^{-1}\left(c_{2}\right) \\
& =\left(c_{1}\right)_{-1} \otimes \varepsilon_{C}\left(\left(c_{1}\right)_{0}\right) \gamma^{-1}\left(c_{2}\right) \\
& \stackrel{(*)}{=} 1_{H} \otimes \varepsilon_{C}\left(c_{1}\right) \gamma^{-1}\left(c_{2}\right)=1_{H} \otimes \gamma^{-1}(c)
\end{aligned}
$$

where in $\left(^{*}\right)$ we used that the comultiplication or the counit of $C$ is left $H$-colinear. Thus $\gamma$ is convolution invertible in ${ }_{H}^{H} \mathcal{Y} \mathcal{D}(C, \mathbb{k})$. The other implication is obvious.

Proposition 2.4. Let $H$ be a Hopf algebra and let $(Q, m, u, \Delta, \varepsilon, \omega)$ be a coquasi-bialgebra in ${ }_{H}^{H} \mathcal{Y} \mathcal{D}$. Let $\gamma: Q \otimes Q \rightarrow \mathbb{k}$ be a gauge transformation in ${ }_{H}^{H} \mathcal{Y} \mathcal{D}$. Then

$$
Q^{\gamma}:=\left(Q, m^{\gamma}, u, \Delta, \varepsilon, \omega^{\gamma}\right)
$$

is a coquasi-bialgebra in ${ }_{H}^{H} \mathcal{Y D}$, where

$$
\begin{aligned}
m^{\gamma} & :=\gamma * m * \gamma^{-1} \\
\omega^{\gamma} & :=(\varepsilon \otimes \gamma) * \gamma(Q \otimes m) * \omega * \gamma^{-1}(m \otimes Q) *\left(\gamma^{-1} \otimes \varepsilon\right) .
\end{aligned}
$$

Proof. The proof is analogue to [K, Proposition XV.3.2] in its dual version. We include some details for the reader's sake. Note that $Q^{\gamma}$ has the same underlying coalgebra of $Q$ which is a coalgebra in ${ }_{H}^{H} \mathcal{Y D}$. The unit is also the same and hence it is a coalgebra map in ${ }_{H}^{H} \mathcal{Y D}$. Since $m^{\gamma}$ is the convolution product of morphisms in ${ }_{H}^{H} \mathcal{Y} \mathcal{D}$, it results that $m^{\gamma}$ is in ${ }_{H}^{H} \mathcal{Y} \mathcal{D}$ as well.

Since $m$ is a coalgebra map in ${ }_{H}^{H} \mathcal{Y D}$ and $\gamma$ is convolution invertible with convolution inverse $\gamma^{-1}$, it follows that $m^{\gamma}$ is a coalgebra map in ${ }_{H}^{H} \mathcal{Y} \mathcal{D}$.

By means of (14) and (15), one gets that $m^{\gamma}\left(1_{Q} \otimes x\right)=x=m^{\gamma}\left(x \otimes 1_{Q}\right)$.

Let us consider now $\omega^{\gamma}$. Since it is the convolution product of morphisms in ${ }_{H}^{H} \mathcal{Y D}$, it results that $\omega^{\gamma}$ is in ${ }_{H}^{H} \mathcal{Y D}$ as well.

Let us check that $\omega^{\gamma}$ is unitary. Consider the map $\alpha_{2}: Q \otimes Q \rightarrow Q \otimes Q \otimes Q$ defined by $\alpha_{2}(x \otimes y)=x \otimes 1_{Q} \otimes y$. The equalities (15) and (6) yield

$$
\begin{aligned}
\left(\alpha_{2}(x \otimes y)\right)_{1} \otimes\left(\alpha_{2}(x \otimes y)\right)_{2} & =\alpha_{2}\left(x_{1} \otimes\left(x_{2}\right)_{-1} y_{1}\right) \otimes \alpha_{2}\left(\left(x_{2}\right)_{0} \otimes y_{2}\right) \\
& =\alpha_{2}\left((x \otimes y)_{1}\right) \otimes \alpha_{2}\left((x \otimes y)_{2}\right)
\end{aligned}
$$

so that $\alpha_{2}$ is comultiplicative.

Thus

$$
\omega^{\gamma} \alpha_{2}:=(\varepsilon \otimes \gamma) \alpha_{2} * \gamma(Q \otimes m) \alpha_{2} * \omega \alpha_{2} * \gamma^{-1}(m \otimes Q) \alpha_{2} *\left(\gamma^{-1} \otimes \varepsilon\right) \alpha_{2}
$$

and computing the factors of this convolution products one gets

$$
\begin{gathered}
(\varepsilon \otimes \gamma) \alpha_{2}=\varepsilon \otimes \varepsilon, \quad \gamma(Q \otimes m) \alpha_{2}=\gamma, \quad \omega \alpha_{2}=\varepsilon \otimes \varepsilon, \\
\gamma^{-1}(m \otimes Q) \alpha_{2}=\gamma^{-1}, \quad\left(\gamma^{-1} \otimes \varepsilon\right) \alpha_{2}=\varepsilon \otimes \varepsilon
\end{gathered}
$$

and hence $\omega^{\gamma} \alpha_{2}=\gamma * \gamma^{-1}=\varepsilon \otimes \varepsilon$, which means that $\omega^{\gamma}\left(x \otimes 1_{Q} \otimes y\right)=\varepsilon(x) \varepsilon(y)$ for every $x, y \in Q$.

Similarly, considering $\alpha_{1}: Q \otimes Q \rightarrow Q \otimes Q \otimes Q$ defined by $\alpha_{2}(x \otimes y)=1_{Q} \otimes x \otimes y$, one proves that $\omega^{\gamma}\left(1_{Q} \otimes x \otimes y\right)=\varepsilon(x) \varepsilon(y)$. A symmetric argument shows that $\omega^{\gamma}\left(x \otimes y \otimes 1_{Q}\right)=\varepsilon(x) \varepsilon(y)$.

Note that, by Lemma 2.3, $\omega^{\gamma}$ is convolution invertible in ${ }_{H}^{H} \mathcal{Y D}(D, \mathbb{k})$ as it is convolution invertible in $\operatorname{Vec}_{\mathbb{k}}(D, \mathbb{k})$.

Let us check that the multiplication is quasi-associative. By ABM, Lemma 2.10 formula (2.7)], we have

$$
m^{\gamma}\left(Q \otimes \gamma * m * \gamma^{-1}\right)=(\varepsilon \otimes \gamma) * m^{\gamma}(Q \otimes m) *\left(\varepsilon \otimes \gamma^{-1}\right),
$$




$$
\begin{aligned}
\left(\varepsilon \otimes \gamma^{-1}\right) *(\varepsilon \otimes \gamma) & =\varepsilon \otimes\left(\gamma^{-1} * \gamma\right)=\varepsilon \otimes \varepsilon \otimes \varepsilon \\
m^{\gamma}\left(m^{\gamma} \otimes Q\right) & =m^{\gamma}\left(\gamma * m * \gamma^{-1} \otimes Q\right)=(\gamma \otimes \varepsilon) * m^{\gamma}\left(m * \gamma^{-1} \otimes Q\right) \\
& =(\gamma \otimes \varepsilon) * m^{\gamma}(m \otimes Q) *\left(\gamma^{-1} \otimes \varepsilon\right) \\
\left(\gamma^{-1} \otimes \varepsilon\right) *(\gamma \otimes \varepsilon) & =\left(\left(\gamma^{-1} * \gamma\right) \otimes \varepsilon\right)=\varepsilon \otimes \varepsilon \otimes \varepsilon .
\end{aligned}
$$

By using these equalities one obtains

$$
\begin{aligned}
& m^{\gamma}\left(Q \otimes m^{\gamma}\right) * \omega^{\gamma}=(\varepsilon \otimes \gamma) * \gamma(Q \otimes m) * m(Q \otimes m) * \omega * \gamma^{-1}(m \otimes Q) *\left(\gamma^{-1} \otimes \varepsilon\right), \\
& \omega^{\gamma} * m^{\gamma}\left(m^{\gamma} \otimes Q\right)=(\varepsilon \otimes \gamma) * \gamma(Q \otimes m) * \omega * m(m \otimes Q) * \gamma^{-1}(m \otimes Q) *\left(\gamma^{-1} \otimes \varepsilon\right)
\end{aligned}
$$

so that $\omega^{\gamma} * m^{\gamma}\left(m^{\gamma} \otimes Q\right)=m^{\gamma}\left(Q \otimes m^{\gamma}\right) * \omega^{\gamma}$.

It remains to check that $\omega^{\gamma}$ is a reassociator. By ABM, Lemma 2.10 formula (2.7)], we have

$$
\begin{aligned}
\omega^{\gamma}\left(Q \otimes Q \otimes \gamma * m * \gamma^{-1}\right) & =(\varepsilon \otimes \varepsilon \otimes \gamma) * \omega^{\gamma}(Q \otimes Q \otimes m) *\left(\varepsilon \otimes \varepsilon \otimes \gamma^{-1}\right), \\
\omega^{\gamma}\left(\gamma * m * \gamma^{-1} \otimes Q \otimes Q\right) & =(\gamma \otimes \varepsilon \otimes \varepsilon) * \omega^{\gamma}(m \otimes Q \otimes Q) *\left(\gamma^{-1} \otimes \varepsilon \otimes \varepsilon\right), \\
(\gamma \otimes \varepsilon \otimes \varepsilon) *(\varepsilon \otimes \varepsilon \otimes \gamma) & =\gamma \otimes \gamma=(\varepsilon \otimes \varepsilon \otimes \gamma) *(\gamma \otimes \varepsilon \otimes \varepsilon) .
\end{aligned}
$$

By using these equalities one obtains

$$
\begin{aligned}
& \omega^{\gamma}\left(Q \otimes Q \otimes m^{\gamma}\right) * \omega^{\gamma}\left(m^{\gamma} \otimes Q \otimes Q\right) \\
& =\left[\begin{array}{c}
(\varepsilon \otimes \varepsilon \otimes \gamma) *(\varepsilon \otimes \gamma(Q \otimes m)) * \gamma(Q \otimes m(Q \otimes m)) \\
* \omega(Q \otimes Q \otimes m) * \omega(m \otimes Q \otimes Q) \\
* \gamma^{-1}(m(m \otimes Q) \otimes Q) *\left(\gamma^{-1}(m \otimes Q) \otimes \varepsilon\right) *\left(\gamma^{-1} \otimes \varepsilon \otimes \varepsilon\right)
\end{array}\right]
\end{aligned}
$$

and

$$
\begin{aligned}
& \left(\varepsilon \otimes \omega^{\gamma}\right) * \omega^{\gamma}\left(Q \otimes m^{\gamma} \otimes Q\right) *\left(\omega^{\gamma} \otimes \varepsilon\right) \\
& =\left[\begin{array}{c}
(\varepsilon \otimes \varepsilon \otimes \gamma) *(\varepsilon \otimes \gamma(Q \otimes m)) * \gamma(Q \otimes m(Q \otimes m)) \\
*(\varepsilon \otimes \omega) * \omega(Q \otimes m \otimes Q) *(\omega \otimes \varepsilon) \\
* \gamma^{-1}(m(m \otimes Q) \otimes Q) *\left(\gamma^{-1}(m \otimes Q) \otimes \varepsilon\right) *\left(\gamma^{-1} \otimes \varepsilon \otimes \varepsilon\right)
\end{array}\right] .
\end{aligned}
$$

Therefore

$$
\omega^{\gamma}\left(Q \otimes Q \otimes m^{\gamma}\right) * \omega^{\gamma}\left(m^{\gamma} \otimes Q \otimes Q\right)=\left(\varepsilon \otimes \omega^{\gamma}\right) * \omega^{\gamma}\left(Q \otimes m^{\gamma} \otimes Q\right) *\left(\omega^{\gamma} \otimes \varepsilon\right) .
$$

In analogy to the case of Hopf algebras, one can define the bosonization $E \# H$ of a coquasibialgebra in ${ }_{H}^{H} \mathcal{Y} \mathcal{D}$ by a Hopf algebra $H$, see [ABM, Definition 5.4] for further details on the structure. The following result was originally stated for $E$ a Hopf algebra. Yorck Sommerhäuser suggested the present more general form which investigates the behaviour of the bosonization under a suitable gauge transformation.

Proposition 2.5. Let $H$ be a Hopf algebra and let $(E, m, u, \Delta, \varepsilon, \omega)$ be a coquasi-bialgebra in ${ }_{H}^{H} \mathcal{Y} \mathcal{D}$. Let $\gamma: E \otimes E \rightarrow \mathbb{k}$ be a gauge transformation in ${ }_{H}^{H} \mathcal{Y} \mathcal{D}$. Set

$$
\Gamma:(E \# H) \otimes(E \# H) \rightarrow \mathbb{k}:(x \# h) \otimes\left(x^{\prime} \# h^{\prime}\right) \mapsto \gamma\left(x \otimes h x^{\prime}\right) \varepsilon_{H}\left(h^{\prime}\right) .
$$

Then $\Gamma$ is a gauge transformation and $(E \# H)^{\Gamma}=E^{\gamma} \# H$ as ordinary coquasi-bialgebras.

Proof. By [ABM, Lemma 2.15 and what follows], we have that $\Gamma$ is convolution invertible $H$-bilinear and $H$-balanced. Moreover $\Gamma^{-1}\left((x \# h) \otimes\left(x^{\prime} \# h^{\prime}\right)\right)=\gamma^{-1}\left(x \otimes h x^{\prime}\right) \varepsilon_{H}\left(h^{\prime}\right)$. If $\alpha:(E \# H) \otimes$ $(E \# H) \rightarrow E \# H$ is $H$-bilinear and $H$-balanced, it is easy to check that $\Gamma * \alpha * \Gamma^{-1}$ is $H$-bilinear and $H$-balanced too.

In particular, since

$$
m_{E \# H}\left((x \# h) \otimes\left(x^{\prime} \# h^{\prime}\right)\right)=m\left(x \otimes h_{1} x^{\prime}\right) \otimes h_{2} h^{\prime}
$$

we have that $m_{E \# H}$ is $H$-bilinear and $H$-balanced where $E \# H$ carries the left $H$-diagonal action and the right regular action over $H$. 
Thus $m_{(E \# H)^{\Gamma}}=\Gamma * m_{E \# H} * \Gamma^{-1}$ is $H$-bilinear and $H$-balanced. Moreover, since $E^{\gamma}$ is also a coquasi-bialgebra in ${ }_{H}^{H} \mathcal{Y} \mathcal{D}$ we have that $m_{E^{\gamma} \# H}:(E \# H) \otimes(E \# H) \rightarrow E \# H$ is $H$-bilinear and $H$-balanced too.

Therefore, in order to check that $m_{(E \# H)^{\Gamma}}=m_{E^{\gamma} \# H}$, it suffices to prove that they coincide on elements of the form $\left(x \# 1_{H}\right) \otimes\left(x^{\prime} \# 1_{H}\right)$.

Let us consider the multiplication

$$
\begin{aligned}
& m_{(E \# H)^{\Gamma}}\left(\left(x \# 1_{H}\right) \otimes\left(x^{\prime} \# 1_{H}\right)\right) \\
= & \left(\Gamma * m_{E \# H} * \Gamma^{-1}\right)\left(\left(x \# 1_{H}\right) \otimes\left(x^{\prime} \# 1_{H}\right)\right) \\
= & \Gamma\left(\left(x \# 1_{H}\right)_{1} \otimes\left(x^{\prime} \# 1_{H}\right)_{1}\right) \cdot m_{E \# H}\left(\left(x \# 1_{H}\right)_{2} \otimes\left(x^{\prime} \# 1_{H}\right)_{2}\right) \cdot \Gamma^{-1}\left(\left(x \# 1_{H}\right)_{3} \otimes\left(x^{\prime} \# 1_{H}\right)_{3}\right) .
\end{aligned}
$$

Now, from

$$
\Delta_{E \# H}(x \# h)=\sum\left(x^{(1)} \# x^{(2)}{ }_{\langle-1\rangle} h_{1}\right) \otimes\left(x^{(2)}{ }_{\langle 0\rangle} \# h_{2}\right)
$$

we get

$$
\begin{aligned}
& \left(x \# 1_{H}\right)_{1} \otimes\left(x \# 1_{H}\right)_{2} \otimes\left(x \# 1_{H}\right)_{3} \\
= & \sum\left(x^{(1)} \# x_{\langle-1\rangle}^{(2)} x_{\langle-2\rangle}^{(3)}\right) \otimes\left(x^{(2)}\langle 0\rangle \# x_{\langle-1\rangle}^{(3)}\right) \otimes\left(x^{(3)}\langle 0\rangle 1_{H}\right)
\end{aligned}
$$

so that

$$
\begin{aligned}
& m_{(E \# H)^{\Gamma}}\left(\left(x \# 1_{H}\right) \otimes\left(x^{\prime} \# 1_{H}\right)\right) \\
& =\quad \Gamma\left(\left(x \# 1_{H}\right)_{1} \otimes\left(x^{\prime} \# 1_{H}\right)_{1}\right) \cdot m_{E \# H}\left(\left(x \# 1_{H}\right)_{2} \otimes\left(x^{\prime} \# 1_{H}\right)_{2}\right) \cdot \Gamma^{-1}\left(\left(x \# 1_{H}\right)_{3} \otimes\left(x^{\prime} \# 1_{H}\right)_{3}\right) \\
& =\left[\begin{array}{c}
\sum \Gamma\left(x^{(1)} \# x^{(2)}\langle-1\rangle x^{(3)}\left\langle(-2\rangle \otimes x^{\prime(1)} \# x^{(2)}\langle-1\rangle x^{\prime(3)}\langle-2\rangle\right)\right. \\
\cdot m_{E \# H}\left(x^{(2)}\langle 0\rangle \# x^{(3)}\langle-1\rangle \otimes x^{\prime(2)}\langle 0\rangle \# x^{\prime(3)}\langle-1\rangle\right) \\
\cdot \Gamma^{-1}\left(x^{(3)}\langle 0\rangle \# 1_{H} \otimes x^{\prime(3)}\langle 0\rangle \# 1_{H}\right)
\end{array}\right] \\
& =\left[\begin{array}{c}
\sum \gamma\left(x^{(1)} \otimes x^{(2)}\langle-1\rangle x^{(3)}\langle-2\rangle x^{(1)}\right) \\
\cdot m_{E \# H}\left(x^{(2)}\langle 0\rangle \# x^{(3)}\langle-1\rangle \otimes x^{\prime(2)} \# x^{\prime(3)}\langle-1\rangle\right) \\
\cdot \gamma^{-1}\left(x^{(3)}\langle 0\rangle \otimes x^{\prime(3)}\langle 0\rangle\right)
\end{array}\right] \\
& =\left[\begin{array}{c}
\sum \gamma\left(x^{(1)} \otimes x^{(2)}\langle-1\rangle x^{(3)}\langle-2\rangle x^{\prime(1)}\right) \\
\cdot m\left(x^{(2)}\langle 0\rangle \otimes x^{(3)}\langle-2\rangle x^{\prime(2)}\right) \otimes x^{(3)}\langle-1\rangle x^{\prime(3)}\langle-1\rangle \\
\cdot \gamma^{-1}\left(x^{(3)}\langle 0\rangle \otimes x^{\prime(3)}\langle 0\rangle\right)
\end{array}\right] \\
& =\left[\begin{array}{c}
\sum \gamma\left(x^{(1)} \otimes x^{(2)}\langle-1\rangle x^{(3)}\langle-2\rangle x^{\prime(1)}\right) \\
\cdot m\left(x^{(2)}\langle 0\rangle \otimes x^{(3)}\langle-1\rangle x^{\prime(2)}\right) \otimes\left(x^{(3)}\langle 0\rangle \otimes x^{\prime(3)}\right)_{\langle-1\rangle} \\
\cdot \gamma^{-1}\left(x^{(3)}\langle 0\rangle \otimes x^{\prime(3)}\langle 0\rangle\right)
\end{array}\right] \\
& \gamma^{-1} \stackrel{\text { colin. }}{=}\left[\begin{array}{c}
\sum \gamma\left(x^{(1)} \otimes x^{(2)}\langle-1\rangle x^{(3)}\langle-2\rangle x^{(1)}\right) \cdot m\left(x^{(2)}\langle 0\rangle \otimes x^{(3)}\langle-1\rangle x^{(2)}\right) \otimes 1_{H} \\
\cdot \gamma^{-1}\left(x^{(3)}\langle 0\rangle \otimes x^{\prime(3)}\right)
\end{array}\right] \\
& =\left[\begin{array}{c}
\sum \gamma\left(x^{(1)} \otimes x^{(2)}\langle-1\rangle x^{(3)}\langle-2\rangle x^{\prime(1)}\right) m\left(x^{(2)}\langle 0\rangle \otimes x^{(3)}\langle-1\rangle x^{\prime(2)}\right) \\
\gamma^{-1}\left(x^{(3)}\langle 0\rangle \otimes x^{\prime(3)}\right)
\end{array}\right] \otimes 1_{H} .
\end{aligned}
$$

Now we have

$$
\sum(x \otimes y)^{(1)} \otimes(x \otimes y)^{(2)}=\sum x^{(1)} \otimes x^{(2)}\langle-1\rangle y^{(1)} \otimes x^{(2)}\langle 0\rangle \otimes y^{(2)}
$$

so that

$$
\begin{aligned}
& \sum(x \otimes y)^{(1)} \otimes(x \otimes y)^{(2)} \otimes(x \otimes y)^{(3)} \\
= & \sum\left(x^{(1)} \otimes x_{\langle-1\rangle}^{(2)} x_{\langle-2\rangle}^{(3)} y^{(1)}\right) \otimes\left(x_{\langle 0\rangle}^{(2)} \otimes x^{(3)}{ }_{\langle-1\rangle} y^{(2)}\right) \otimes\left(x^{(3)}\langle 0\rangle \otimes y^{(3)}\right) .
\end{aligned}
$$

Using this equality we can proceed in our computation:

$$
m_{(E \# H)^{\Gamma}}\left(\left(x \# 1_{H}\right) \otimes\left(x^{\prime} \# 1_{H}\right)\right)
$$


COHOMOLOGY AND COQUASI-BIALGEBRAS IN THE CATEGORY OF YETTER-DRINFELD MODULES

$$
\begin{aligned}
& =\left[\begin{array}{c}
\sum \gamma\left(x ^ { ( 1 ) } \otimes x ^ { ( 2 ) } \left\langle-1 x^{(3)}\left\langle-2 x^{\prime(1)}\right)\right.\right. \\
m\left(x^{(2)}\langle 0\rangle \otimes x^{(3)}\langle-1\rangle x^{\prime(2)}\right) \gamma^{-1}\left(x^{(3)}{ }_{\langle 0\rangle} \otimes x^{\prime(3)}\right)
\end{array}\right] \otimes 1_{H} \\
& =\left[\sum \gamma\left(\left(x \otimes x^{\prime}\right)^{(1)}\right) \cdot m\left(\left(x \otimes x^{\prime}\right)^{(2)}\right) \cdot \gamma^{-1}\left(\left(x \otimes x^{\prime}\right)^{(3)}\right)\right] \# 1_{H} \\
& =\left(\gamma * m * \gamma^{-1}\right)\left(x \otimes x^{\prime}\right) \# 1_{H} \\
& =m_{E \gamma}\left(x \otimes x^{\prime}\right) \# 1_{H} \\
& =m_{E \gamma \# H}\left(\left(x \# 1_{H}\right) \otimes\left(x^{\prime} \# 1_{H}\right)\right) \text {. }
\end{aligned}
$$

Finally $u_{(E \# H)^{\Gamma}}=u_{E \# H}=1_{E} \# 1_{H}=1_{E \gamma} \# 1_{H}=u_{E \gamma \# H}$.

As a coalgebra $(E \# H)^{\Gamma}$ coincides with $E \# H$ and hence with $E^{\gamma} \# H$.

Finally let us check that $\omega_{E^{\gamma} \# H}$ and $\omega_{(E \# H)^{\Gamma}}$ coincide. To this aim, let us use the maps $\mho_{H,-}^{*}$ of ABM, Lemma 2.15]. First note that $\omega_{E^{\gamma} \# H}=\mho_{H, E^{\gamma}}^{3}\left(\omega_{E^{\gamma}}\right)$ by [ABM, Proposition 5.3]. Now

$$
\begin{aligned}
\omega_{(E \# H)^{\Gamma}=} & \left(\varepsilon_{E \# H} \otimes \Gamma\right) * \Gamma\left(E \# H \otimes m_{E \# H}\right) * \omega_{E \# H} * \Gamma^{-1}\left(m_{E \# H} \otimes E \# H\right) *\left(\Gamma^{-1} \otimes \varepsilon_{E \# H}\right) \\
= & \left(\mho_{H, E}^{1}(\varepsilon) \otimes \mho_{H, E}^{2}(\gamma)\right) * \mho_{H, E}^{2}(\gamma)\left(E \# H \otimes m_{E \# H}\right) * \mho_{H, E}^{3}(\omega) \\
& * \mho_{H, E}^{2}\left(\gamma^{-1}\right)\left(m_{E \# H} \otimes E \# H\right) *\left(\mho_{H, E}^{2}\left(\gamma^{-1}\right) \otimes \mho_{H, E}^{1}(\varepsilon)\right)
\end{aligned}
$$

One easily checks that

$$
\begin{aligned}
\mho_{H, E}^{1}(\varepsilon) \otimes \mho_{H, E}^{2}(\gamma) & =\mho_{H, E^{\gamma}}^{3}(\varepsilon \otimes \gamma), \\
\mho_{H, E}^{2}(\gamma)\left(E \# H \otimes m_{E \# H}\right) & =\mho_{H, E^{\gamma}}^{3}(\gamma(E \otimes m)), \\
\mho_{H, E}^{2}\left(\gamma^{-1}\right)\left(m_{E \# H} \otimes E \# H\right) & =\mho_{H, E^{\gamma}}^{3}\left(\gamma^{-1}(m \otimes E)\right), \\
\mho_{H, E}^{2}\left(\gamma^{-1}\right) \otimes \mho_{H, E}^{1}\left(\varepsilon_{E}\right) & =\mho_{H, E^{\gamma}}^{3}\left(\gamma^{-1} \otimes \varepsilon\right) .
\end{aligned}
$$

Thus we obtain

$$
\begin{aligned}
\omega_{(E \# H)^{\Gamma}} & =\mho_{H, E^{\gamma}}^{3}(\varepsilon \otimes \gamma) * \mho_{H, E \gamma}^{3}(\gamma(E \otimes m)) * \mho_{H, E}^{3}(\omega) * \mho_{H, E^{\gamma}}^{3}\left(\gamma^{-1}(m \otimes E)\right) * \mho_{H, E \gamma}^{3}\left(\gamma^{-1} \otimes \varepsilon\right) \\
& =\mho_{H, E^{\gamma}}^{3}\left[(\varepsilon \otimes \gamma) * \gamma(E \otimes m) * \omega * \gamma^{-1}(m \otimes E) *\left(\gamma^{-1} \otimes \varepsilon\right)\right] \\
& =\mho_{H, E^{\gamma}}^{3}\left(\omega_{E \gamma}\right)=\omega_{E \gamma \# H} .
\end{aligned}
$$

Proposition 2.6. Let $H$ be a Hopf algebra and let $(Q, m, u, \Delta, \varepsilon, \omega)$ be a connected coquasibialgebra in ${ }_{H}^{H} \mathcal{Y D}$. Let $\gamma: Q \otimes Q \rightarrow \mathbb{k}$ be a gauge transformation in ${ }_{H}^{H} \mathcal{Y} \mathcal{D}$. Then $\operatorname{gr}\left(Q^{\gamma}\right)$ and $\operatorname{gr}(Q)$ coincide as bialgebras in ${ }_{H}^{H} \mathcal{Y D}$.

Proof. By Proposition 2.4. $Q^{\gamma}$ is a coquasi-bialgebra in ${ }_{H}^{H} \mathcal{Y D}$. It is obviously connected as it coincides with $Q$ as a coalgebra. By Theorem 1.5, both $\operatorname{gr} Q$ and $\operatorname{gr}\left(Q^{\gamma}\right)$ are connected bialgebras in ${ }_{H}^{H} \mathcal{Y D}$. Let us check they coincide.

Note that, by Remark 2.2, we have that $\gamma^{-1}$ is a gauge transformation, hence it is trivial on $\mathbb{k} 1_{Q} \otimes 1_{Q}$. Let $C:=Q \otimes Q$. Let $n>0$ and let $w \in C_{(n)}=\sum_{i+j<n} Q_{i} \otimes Q_{j}$. By AMS1, Lemma 3.69], we have that $\Delta_{C}(w)-w \otimes\left(1_{Q}\right)^{\otimes 2}-\left(1_{Q}\right)^{\otimes 2} \otimes w \in C_{(n-1)} \otimes C_{(n-1)}$. Thus we get

$$
w_{1} \otimes w_{2} \otimes w_{3}-\Delta_{C}(w) \otimes\left(1_{Q}\right)^{\otimes 2}-\Delta_{C}\left(\left(1_{Q}\right)^{\otimes 2}\right) \otimes w \in \Delta_{C}\left(C_{(n-1)}\right) \otimes C_{(n-1)}
$$

and hence

$w_{1} \otimes w_{2} \otimes w_{3}-w \otimes\left(1_{Q}\right)^{\otimes 2} \otimes\left(1_{Q}\right)^{\otimes 2}-\left(1_{Q}\right)^{\otimes 2} \otimes w \otimes\left(1_{Q}\right)^{\otimes 2}-\left(1_{Q}\right)^{\otimes 4} \otimes w \in C_{(n-1)} \otimes C_{(n-1)} \otimes C_{(n-1)}$.

Since $m\left(C_{(n-1)}\right) \subseteq Q_{n-1}$ we get

$w_{1} \otimes m\left(w_{2}\right) \otimes w_{3}-w \otimes 1_{Q} \otimes\left(1_{Q}\right)^{\otimes 2}-\left(1_{Q}\right)^{\otimes 2} \otimes m(w) \otimes\left(1_{Q}\right)^{\otimes 2}-\left(1_{Q}\right)^{\otimes 3} \otimes w \in C_{(n-1)} \otimes Q_{n-1} \otimes C_{(n-1)}$

and hence

$$
w_{1} \otimes\left(m\left(w_{2}\right)+Q_{n-1}\right) \otimes w_{3}=\left(1_{Q}\right)^{\otimes 2} \otimes\left(m(w)+Q_{n-1}\right) \otimes\left(1_{Q}\right)^{\otimes 2} .
$$


Let $x, y \in Q$. We compute

$$
\begin{aligned}
\bar{x} \cdot \gamma \cdot \bar{y} & =\left(x+Q_{|x|-1}\right) \cdot \gamma\left(y+Q_{|y|-1}\right) \\
& =(x \cdot \gamma y)+Q_{|x|+|y|-1} \\
& =\gamma\left((x \otimes y)_{1}\right) m\left((x \otimes y)_{2}\right) \gamma^{-1}\left((x \otimes y)_{3}\right)+Q_{|x|+|y|-1} \\
& =\gamma\left((x \otimes y)_{1}\right)\left(m\left((x \otimes y)_{2}\right)+Q_{|x|+|y|-1}\right) \gamma^{-1}\left((x \otimes y)_{3}\right) \\
& =\gamma\left(\left(1_{Q}\right)^{\otimes 2}\right)\left(m(x \otimes y)+Q_{|x|+|y|-1}\right) \gamma^{-1}\left(\left(1_{Q}\right)^{\otimes 2}\right) \\
& =m(x \otimes y)+Q_{|x|+|y|-1}=(x \cdot y)+Q_{|x|+|y|-1}=\bar{x} \cdot \bar{y} .
\end{aligned}
$$

Note that $Q^{\gamma}$ and $Q$ have the same unit so that $\operatorname{gr} Q$ and $\operatorname{gr} Q^{\gamma}$ have.

\section{3. (CO)SEMisimple CASE}

Assume $H$ is a semisimple and cosemisimple Hopf algebra (e.g. $H$ is finite-dimensional cosemisimple over a field of characteristic zero). Note that $H$ is then separable (see e.g. [Stt, Corollary 3.7] or AMS1, Theorem 2.34]) whence finite-dimensional. Let $(Q, m, u, \Delta, \varepsilon)$ be a f.d. coalgebra with multiplication and unit in ${ }_{H}^{H} \mathcal{Y D}$. Assume that the coradical $Q_{0}$ is a subcoalgebra of $Q$ in ${ }_{H}^{H} \mathcal{Y D}$ such that $Q_{0} \cdot Q_{0} \subseteq Q_{0}$. Let $y^{n, i}$ with $1 \leq i \leq \operatorname{dim}\left(Q_{n} / Q_{n-1}\right)$ be a basis for $Q_{n} / Q_{n-1}$. Consider, for every $n>0$, the exact sequence in ${ }_{H}^{H} \mathcal{Y D}$ given by

$$
0 \longrightarrow Q_{n-1} \stackrel{s_{n}}{\longrightarrow} Q_{n} \stackrel{\pi_{n}}{\longrightarrow} \frac{Q_{n}}{Q_{n-1}} \longrightarrow 0
$$

Now, since $H$ is semisimple and cosemisimple, by [Ra2, Proposition 7] the Drinfeld double $D(H)$ is semisimple. By a result essentially due to Majid (see [Md, Proposition 10.6.16]) and by [RT, Proposition 6], we get that the category ${ }_{H}^{H} \mathcal{Y D} \cong{ }_{D(H)} \mathfrak{M}$ is a semisimple category. Therefore $\pi_{n}$ cosplits i.e. there is a morphism $\sigma_{n}:\left(Q_{n} / Q_{n-1}\right) \rightarrow Q_{n}$ in ${ }_{H}^{H} \mathcal{Y D}$ such that $\pi_{n} \sigma_{n}=$ Id. Let $u_{n}: \mathbb{k} \rightarrow Q_{n}$ be the corestriction of the unit $u: \mathbb{k} \rightarrow Q$ and let $\varepsilon_{n}=\varepsilon_{\mid Q_{n}}: Q_{n} \rightarrow \mathbb{k}$ be the counit of the subcoalgebra $Q_{n}$. Set

This is a morphism in ${ }_{H}^{H} \mathcal{Y} \mathcal{D}$. Moreover

$$
\sigma_{n}^{\prime}:=\sigma_{n}-u_{n} \circ \varepsilon_{n} \circ \sigma_{n}
$$

$$
\begin{aligned}
& \pi_{n} \circ \sigma_{n}^{\prime}=\pi_{n} \circ \sigma_{n}-\pi_{n} \circ u_{n} \circ \varepsilon_{n} \circ \sigma_{n} \stackrel{n \geq 0}{=} \operatorname{Id}_{Q_{n} / Q_{n-1}}-0=\operatorname{Id}_{Q_{n} / Q_{n-1}}, \\
& \varepsilon_{n} \circ \sigma_{n}^{\prime}=\varepsilon_{n} \circ \sigma_{n}-\varepsilon_{n} \circ u_{n} \circ \varepsilon_{n} \circ \sigma_{n}=\varepsilon_{n} \circ \sigma_{n}-\varepsilon_{n} \circ \sigma_{n}=0 .
\end{aligned}
$$

Therefore, without loss of generality we can assume that $\varepsilon_{n} \circ \sigma_{n}=0$. A standard argument on split short exact sequences shows that there exists a morphism $p_{n}: Q_{n} \rightarrow Q_{n-1}$ in ${ }_{H}^{H} \mathcal{Y D}$ such that $s_{n} p_{n}+\sigma_{n} \pi_{n}=\operatorname{Id}_{Q_{n}}, p_{n} s_{n}=\operatorname{Id}_{Q_{n-1}}$ and $p_{n} \sigma_{n}=0$. We set

$$
x^{n, i}:=\sigma_{n}\left(y^{n, i}\right) \text {. }
$$

Therefore

$$
y^{n, i}=\pi_{n} \sigma_{n}\left(y^{n, i}\right)=\pi_{n}\left(x^{n, i}\right)=x^{n, i}+Q_{n-1}=\overline{x^{n, i}} .
$$

These terms $x^{n, i}$ define a $\mathbb{k}$-basis for $Q$. As $Q$ is finite-dimensional, there exists $d \in \mathbb{N}_{0}$ such that $Q=Q_{d}$; we fix $d$ minimal. For all $0 \leq a \leq b$, define the maps

$$
\begin{aligned}
& p_{a, b}: \quad Q_{b} \rightarrow Q_{a}, \quad p_{a, b}:=p_{a+1} \circ p_{a+2} \circ \cdots \circ p_{b-1} \circ p_{b}, \\
& s_{b, a}: Q_{a} \rightarrow Q_{b}, \quad s_{b, a}:=s_{b} \circ s_{b-1} \circ \cdots \circ s_{a+2} \circ s_{a+1} .
\end{aligned}
$$

Clearly one has

Thus, for $0 \leq i, a \leq b$ we have

$$
p_{a, b} \circ s_{b, a}=\operatorname{Id}_{Q_{a}} .
$$

$$
p_{i, b} \circ s_{b, a}=\left\{\begin{array}{cc}
p_{i, b} \circ s_{b, i} \circ s_{i, a} & i>a \\
p_{i, a} \circ p_{a, b} \circ s_{b, a} & i \leq a
\end{array}= \begin{cases}s_{i, a} & i>a \\
p_{i, a} & i \leq a\end{cases}\right.
$$

Thus we get an isomorphism $\varphi: Q \rightarrow \operatorname{gr} Q$ of objects in ${ }_{H}^{H} \mathcal{Y D}$ given by

$$
\varphi(x):=\quad p_{0, d}(x)+\pi_{1} p_{1, d}(x)+\pi_{2} p_{2, d}(x)+\cdots+\pi_{d-2} p_{d-2, d}(x)+\pi_{d-1} p_{d-1, d}(x)+\pi_{d}(x)
$$


COHOMOLOGY AND COQUASI-BIALGEBRAS IN THE CATEGORY OF YETTER-DRINFELD MODULES 11

where we set

$$
=\sum_{0 \leq t \leq d} \pi_{t} p_{t, d}(x), \text { for every } x \in Q,
$$

For $0 \leq n \leq d$, we have

$$
\pi_{0}=\operatorname{Id}_{Q_{0}}, \quad p_{d, d}=\operatorname{Id}_{Q_{d}} .
$$

$$
\begin{aligned}
\varphi\left(x^{n, i}\right) & =\varphi\left(s_{d, n}\left(x^{n, i}\right)\right)=\varphi\left(s_{d, n} \sigma_{n}\left(y^{n, i}\right)\right)=\sum_{0 \leq t \leq d} \pi_{t} p_{t, d} s_{d, n}\left(\sigma_{n}\left(y^{n, i}\right)\right) \\
& =\sum_{n<t \leq d} \pi_{t} p_{t, d} s_{d, n}\left(\sigma_{n}\left(y^{n, i}\right)\right)+\sum_{0 \leq t \leq n} \pi_{t} p_{t, d} s_{d, n}\left(\sigma_{n}\left(y^{n, i}\right)\right) \\
& =\sum_{n<t \leq d} \pi_{t} s_{t, n}\left(\sigma_{n}\left(y^{n, i}\right)\right)+\sum_{0 \leq t<n} \pi_{t} p_{t, n}\left(\sigma_{n}\left(y^{n, i}\right)\right)+\pi_{n} p_{n, d} s_{d, n}\left(\sigma_{n}\left(y^{n, i}\right)\right) \\
= & \sum_{n<t \leq d} \pi_{t} s_{t, t-1} s_{t-1, n}\left(\sigma_{n}\left(y^{n, i}\right)\right)+\sum_{0 \leq t<n} \pi_{t} p_{t, n-1} p_{n-1, n}\left(\sigma_{n}\left(y^{n, i}\right)\right)+ \\
= & \sum_{n<t \leq d}{\pi_{n, d}}_{d, n}\left(\sigma_{n}\left(y^{n, i}\right)\right) \\
= & 0+0+y^{n, i}=y^{n, i} .
\end{aligned}
$$

Hence $\varphi\left(x^{n, i}\right)=y^{n, i}$. Since $y^{n, i}$ with $1 \leq i \leq \operatorname{dim}\left(Q_{n} / Q_{n-1}\right)=: d_{n}$ form a basis for $Q_{n} / Q_{n-1}$ we have that

$$
h y^{n, i} \in \frac{Q_{n}}{Q_{n-1}}, \quad\left(y^{n, i}\right)_{-1} \otimes\left(y^{n, i}\right)_{0} \in H \otimes \frac{Q_{n}}{Q_{n-1}} .
$$

Therefore there are $\chi_{t, i}^{n} \in H^{*}$ and $h_{t, i}^{n} \in H$ such that

$$
h y^{n, i}=\sum_{1 \leq t \leq d_{n}} \chi_{t, i}^{n}(h) y^{n, t}, \quad\left(y^{n, i}\right)_{-1} \otimes\left(y^{n, i}\right)_{0}=\sum_{1 \leq t \leq d_{n}} h_{i, t}^{n} \otimes y^{n, t} .
$$

We have

$$
\begin{aligned}
h\left(h^{\prime} y^{n, i}\right) & =\sum_{1 \leq s \leq d_{n}} \chi_{s, i}^{n}\left(h^{\prime}\right) h y^{n, s}=\sum_{1 \leq s \leq d_{n}} \chi_{s, i}^{n}\left(h^{\prime}\right) \sum_{1 \leq t \leq d_{n}} \chi_{t, s}^{n}(h) y^{n, t} \\
& =\sum_{1 \leq s \leq d_{n}} \sum_{1 \leq t \leq d_{n}} \chi_{t, s}^{n}(h) \chi_{s, i}^{n}\left(h^{\prime}\right) y^{n, t}, \\
\left(h h^{\prime}\right) y^{n, i} & =\sum_{1 \leq t \leq d_{n}} \chi_{t, i}^{n}\left(h h^{\prime}\right) y^{n, t}
\end{aligned}
$$

and hence

Moreover

$$
\chi_{t, i}^{n}\left(h h^{\prime}\right)=\sum_{1 \leq s \leq d_{n}} \chi_{t, s}^{n}(h) \chi_{s, i}^{n}\left(h^{\prime}\right) .
$$

and hence

We also have

$$
y^{n, i}=1_{H} y^{n, i}=\sum_{1 \leq t \leq d_{n}} \chi_{t, i}^{n}\left(1_{H}\right) y^{n, t}
$$

$$
\chi_{t, i}^{n}\left(1_{H}\right)=\delta_{t, i}
$$

$$
\begin{aligned}
\left(y^{n, i}\right)_{-1} \otimes\left(\left(y^{n, i}\right)_{0}\right)_{-1} \otimes\left(\left(y^{n, i}\right)_{0}\right)_{0} & =\sum_{1 \leq s \leq d_{n}} h_{i, s}^{n} \otimes\left(y^{n, s}\right)_{-1} \otimes\left(y^{n, s}\right)_{0} \\
& =\sum_{1 \leq s \leq d_{n}} h_{i, s}^{n} \otimes \sum_{1 \leq t \leq d_{n}} h_{s, t}^{n} \otimes y^{n, t} \\
& =\sum_{1 \leq s \leq d_{n}} \sum_{1 \leq t \leq d_{n}} h_{i, s}^{n} \otimes h_{s, t}^{n} \otimes y^{n, t}, \\
\left(\left(y^{n, i}\right)_{-1}\right)_{1} \otimes\left(\left(y^{n, i}\right)_{-1}\right)_{2} \otimes\left(y^{n, i}\right)_{0} & =\sum_{1 \leq t \leq d_{n}} \Delta_{H}\left(h_{t, i}^{n}\right) \otimes y^{n, t}
\end{aligned}
$$

so that

Moreover

$$
\Delta_{H}\left(h_{t, i}^{n}\right)=\sum_{1 \leq s \leq d_{n}} h_{i, s}^{n} \otimes h_{s, t}^{n} .
$$

and hence

$$
y^{n, i}=\varepsilon_{H}\left(\left(y^{n, i}\right)_{-1}\right)\left(y^{n, i}\right)_{0}=\sum_{1 \leq t \leq d_{n}} \varepsilon_{H}\left(h_{t, i}^{n}\right) y^{n, t}
$$

$$
\varepsilon_{H}\left(h_{t, i}^{n}\right)=\delta_{t, i} .
$$


Finally

$$
\begin{aligned}
\left(h_{1} y^{n, i}\right)_{-1} h_{2} \otimes\left(h_{1} y^{n, i}\right)_{0} & =\sum_{1 \leq s \leq d_{n}} \chi_{s, i}^{n}\left(h_{1}\right)\left(y^{n, s}\right)_{-1} h_{2} \otimes\left(y^{n, s}\right)_{0} \\
& =\sum_{1 \leq s \leq d_{n}} \chi_{s, i}^{n}\left(h_{1}\right) \sum_{1 \leq t \leq d_{n}} h_{s, t}^{n} h_{2} \otimes y^{n, t} \\
& =\sum_{1 \leq s \leq d_{n}} \sum_{1 \leq t \leq d_{n}} h_{s, t}^{n} \chi_{s, i}^{n}\left(h_{1}\right) h_{2} \otimes y^{n, t}, \\
h_{1}\left(y^{n, i}\right)_{-1} \otimes h_{2}\left(y^{n, i}\right)_{0} & =\sum_{1 \leq s \leq d_{n}} h_{1} h_{i, s}^{n} \otimes h_{2} y^{n, s}=\sum_{1 \leq s \leq d_{n}} h_{1} h_{i, s}^{n} \otimes \sum_{1 \leq t \leq d_{n}} \chi_{t, s}^{n}\left(h_{2}\right) y^{n, t} \\
& =\sum_{1 \leq s \leq d_{n}} \sum_{1 \leq t \leq d_{n}} h_{1} \chi_{t, s}^{n}\left(h_{2}\right) h_{i, s}^{n} \otimes y^{n, t}
\end{aligned}
$$

Therefore, we get

We have

$$
\sum_{1 \leq s \leq d_{n}} h_{s, t}^{n} \chi_{s, i}^{n}\left(h_{1}\right) h_{2}=\sum_{1 \leq s \leq d_{n}} h_{1} \chi_{t, s}^{n}\left(h_{2}\right) h_{i, s}^{n} .
$$

$$
\begin{aligned}
h x^{n, i} & =h \sigma_{n}\left(y^{n, i}\right)=\sigma_{n}\left(h y^{n, i}\right)=\sigma_{n}\left(\sum_{1 \leq t \leq d_{n}} \chi_{t, i}^{n}(h) y^{n, t}\right)=\sum_{1 \leq t \leq d_{n}} \chi_{t, i}^{n}(h) x^{n, t}, \\
\left(x^{n, i}\right)_{-1} \otimes\left(x^{n, i}\right)_{0} & =\left(\sigma_{n}\left(y^{n, i}\right)\right)_{-1} \otimes\left(\sigma_{n}\left(y^{n, i}\right)\right)_{0}=\left(y^{n, i}\right)_{-1} \otimes \sigma_{n}\left(\left(y^{n, i}\right)_{0}\right)=\sum_{1 \leq t \leq d_{n}} h_{i, t}^{n} \otimes x^{n, t}, \\
\varepsilon_{Q}\left(x^{n, i}\right) & =\varepsilon_{n}\left(x^{n, i}\right)=\varepsilon_{n} \sigma_{n}\left(y^{n, i}\right)=0 \text { for } n>0 .
\end{aligned}
$$

If $Q$ is connected, then $d_{0}=1$ so we may assume $y^{0,0}:=1_{Q}+Q_{-1}$. Since $\pi_{0}=\operatorname{Id}_{Q_{0}}$ we get

$$
\sigma_{0}=\operatorname{Id}_{Q_{0}} \circ \sigma_{0}=\pi_{0} \circ \sigma_{0}=\operatorname{Id}_{Q_{0}}
$$

and hence

$$
x^{0,0}=\sigma_{0}\left(y^{0,0}\right)=\sigma_{0}\left(1_{Q}+Q_{-1}\right)=1_{Q} .
$$

Since, by Proposition 1.3. $Q_{a} \cdot Q_{a^{\prime}} \subseteq Q_{a+a^{\prime}}$ for every $a, a^{\prime} \in \mathbb{N}_{0}$, we can write the product of two elements of the basis in the form

$$
x^{a, l} x^{a^{\prime}, l^{\prime}}=\sum_{u \leq a+a^{\prime}} \sum_{v} \mu_{u, v}^{a, l, a^{\prime}, l^{\prime}} x^{u, v} .
$$

We compute

$$
\begin{aligned}
\overline{x^{a, l}} \cdot \overline{x^{a^{\prime}, l^{\prime}}}= & \left(x^{a, l}+Q_{a-1}\right)\left(x^{a^{\prime}, l^{\prime}}+Q_{a^{\prime}-1}\right) \\
= & \left(x^{a, l} x^{a^{\prime}, l^{\prime}}\right)+Q_{a+a^{\prime}-1} \\
& \stackrel{99}{=}\left(\sum_{u \leq a+a^{\prime}} \sum_{v} \mu_{u, v}^{a, l, a^{\prime}, l^{\prime}} x^{u, v}\right)+Q_{a+a^{\prime}-1} \\
= & \left(\sum_{v} \mu_{a+a^{\prime}, v}^{a, l, a^{\prime}, l^{\prime}} x^{a+a^{\prime}, v}\right)+Q_{a+a^{\prime}-1} \\
= & \sum_{v} \mu_{a+a^{\prime}, v}^{a, l, l^{\prime}, l^{\prime}}\left(x^{a+a^{\prime}, v}+Q_{a+a^{\prime}-1}\right) \\
= & \sum_{v} \mu_{a+a^{\prime}, v}^{a, l, a^{\prime},} \frac{x^{a+a^{\prime}, v}}{}
\end{aligned}
$$

which gives

$$
\overline{x^{a, l}} \cdot \overline{x^{a^{\prime}, l^{\prime}}}=\sum_{v} \mu_{a+a^{\prime}, v}^{a, l, a^{\prime}, l^{\prime}} \overline{x^{a+a^{\prime}, v}} .
$$

Remark 3.1. Let $H$ be a Hopf algebra and let $\left(A, m_{A}, u_{A}\right)$ be an algebra in ${ }_{H}^{H} \mathcal{Y D}$. Let $\varepsilon_{A}: A \rightarrow \mathbb{k}$ be an algebra map in ${ }_{H}^{H} \mathcal{Y D}$. The Hochschild cohomology in a monoidal category is known, see e.g. AMS2]. Consider $\mathbb{k}$ as an $A$-bimodule in ${ }_{H}^{H} \mathcal{Y D}$ through $\varepsilon_{A}$. Then, following [AMS2, 1.24], we can consider an analogue of the standard complex

$$
{ }_{H}^{H} \mathcal{Y D}(\mathbb{k}, \mathbb{k}) \stackrel{\partial^{0}}{\longrightarrow}{ }_{H}^{H} \mathcal{Y} \mathcal{D}(A, \mathbb{k}) \stackrel{\partial^{1}}{\longrightarrow}{ }_{H}^{H} \mathcal{Y} \mathcal{D}\left(A^{\otimes 2}, \mathbb{k}\right) \stackrel{\partial^{2}}{\longrightarrow}{ }_{H}^{H} \mathcal{Y} \mathcal{D}\left(A^{\otimes 3}, \mathbb{k}\right) \stackrel{\partial^{3}}{\longrightarrow} \cdots
$$

Explicitly, given $f$ in the corresponding domain of $\partial^{n}$, for $n=0,1,2,3$, we have

$$
\partial^{0}(f)=f(1) \varepsilon_{A}-\varepsilon_{A} f(1)=0,
$$




$$
\begin{aligned}
& \partial^{1}(f)=f \otimes \varepsilon_{A}-f m_{A}+\varepsilon_{A} \otimes f \\
& \partial^{2}(f)=f \otimes \varepsilon_{A}-f\left(A \otimes m_{A}\right)+f\left(m_{A} \otimes A\right)-\varepsilon_{A} \otimes f \\
& \partial^{3}(f)=f \otimes \varepsilon_{A}-f\left(A \otimes A \otimes m_{A}\right)+f\left(A \otimes m_{A} \otimes A\right)-f\left(m_{A} \otimes A \otimes A\right)+\varepsilon_{A} \otimes f .
\end{aligned}
$$

For every $n \geq 1$ denote by

$$
\mathrm{Z}_{\mathcal{Y} \mathcal{D}}^{n}(A, \mathbb{k}):=\operatorname{ker}\left(\partial^{n}\right), \quad \mathrm{B}_{\mathcal{Y} \mathcal{D}}^{n}(A, \mathbb{k}):=\operatorname{Im}\left(\partial^{n-1}\right) \quad \text { and } \quad \mathrm{H}_{\mathcal{Y} \mathcal{D}}^{n}(A, \mathbb{k}):=\frac{\mathrm{Z}_{\mathcal{Y} \mathcal{D}}^{n}(A, \mathbb{k})}{\mathrm{B}_{\mathcal{Y} \mathcal{D}}^{n}(A, \mathbb{k})}
$$

the abelian groups of $n$-cocycles, of $n$-coboundaries and the $n$-th Hochschild cohomology group in ${ }_{H}^{H} \mathcal{Y D}$ of the algebra $A$ with coefficients in $\mathbb{k}$. We point out that the construction above works for an arbitrary $A$-bimodule $M$ in ${ }_{H}^{H} \mathcal{Y D}$ instead of $\mathbb{k}$.

Next result is inspired by [EG, Proposition 2.3]. Two coquasi-bialgebras $Q$ and $Q^{\prime}$ in ${ }_{H}^{H} \mathcal{Y D}$ will be called gauge equivalent whenever there is some gauge transformation $\gamma: Q \otimes Q \rightarrow \mathbb{k}$ in ${ }_{H}^{H} \mathcal{Y} \mathcal{D}$ such that $Q^{\gamma} \cong Q^{\prime}$ as coquasi-bialgebras in ${ }_{H}^{H} \mathcal{Y} \mathcal{D}$, see Proposition 2.4 for the structure of $Q^{\gamma}$.

TheOREM 3.2. Let $H$ be a semisimple and cosemisimple Hopf algebra and let $(Q, m, u, \Delta, \varepsilon, \omega)$ be a f.d. connected coquasi-bialgebra in ${ }_{H}^{H} \mathcal{Y D}$. If $\mathrm{H}_{\mathcal{Y} \mathcal{D}}^{3}(\operatorname{gr} Q, \mathbb{k})=0$ then $Q$ is gauge equivalent to a connected bialgebra in ${ }_{H}^{H} \mathcal{Y D}$.

Proof. For $t \in \mathbb{N}_{0}$, and $x, y, z$ in the basis of $Q$, we set

$$
\omega_{t}(x \otimes y \otimes z):=\delta_{|x|+|y|+|z|, t} \omega(x \otimes y \otimes z) .
$$

Let us check it defines a morphism $\omega_{t}: Q \otimes Q \otimes Q \rightarrow \mathbb{k}$ in ${ }_{H}^{H} \mathcal{Y} \mathcal{D}$. It is left $H$-linear as, by means of (18), the definition of $\omega_{t}$ and the $H$-linearity of $\omega$, we can prove that $\omega_{t}\left(h\left(x^{n, i} \otimes x^{n^{\prime}, i^{\prime}} \otimes x^{n^{\prime \prime}, i^{\prime \prime}}\right)\right)=$ $\varepsilon_{H}(h) \omega_{t}\left(x^{n, i} \otimes x^{n^{\prime}, i^{\prime}} \otimes x^{n^{\prime \prime}, i^{\prime \prime}}\right)$.

Moreover it is left $H$-colinear as, by means of (18), the definition of $\omega_{t}$ and the $H$-colinearity of $\omega$, we can prove that

$$
\left(x^{n, i} \otimes x^{n^{\prime}, i^{\prime}} \otimes x^{n^{\prime \prime}, i^{\prime \prime}}\right)_{\langle-1\rangle} \otimes \omega_{t}\left(\left(x^{n, i} \otimes x^{n^{\prime}, i^{\prime}} \otimes x^{n^{\prime \prime}, i^{\prime \prime}}\right)_{\langle 0\rangle}\right)=1_{H} \otimes \omega_{t}\left(x^{n, i} \otimes x^{n^{\prime}, i^{\prime}} \otimes x^{n^{\prime \prime}, i^{\prime \prime}}\right) .
$$

Clearly, for $x, y, z \in Q$ in the basis, one has

$$
\sum_{t \in \mathbb{N}_{0}} \omega_{t}(x \otimes y \otimes z)=\sum_{t \in \mathbb{N}_{0}} \delta_{|x|+|y|+|z|, t} \omega(x \otimes y \otimes z)=\omega(x \otimes y \otimes z)
$$

so that we can formally write

$$
\omega=\sum_{t \in \mathbb{N}_{0}} \omega_{t}
$$

Since $\varepsilon$ is trivial on elements in the basis of strictly positive degree, one gets

$$
\omega_{0}=\varepsilon \otimes \varepsilon \otimes \varepsilon .
$$

If $\omega=\omega_{0}$ then $Q$ is a (connected) bialgebra in ${ }_{H}^{H} \mathcal{Y D}$ and the proof is finished. Thus we can assume $\omega \neq \omega_{0}$ and set

$$
\begin{aligned}
& s \quad:=\min \left\{i \in \mathbb{N}: \omega_{i} \neq 0\right\}, \\
& \bar{\omega}_{s} \quad: \quad=\omega_{s}\left(\varphi^{-1} \otimes \varphi^{-1} \otimes \varphi^{-1}\right), \\
& \bar{Q} \quad: \quad=\operatorname{gr} Q .
\end{aligned}
$$

Note that $\bar{\omega}_{s}$ is a morphism in ${ }_{H}^{H} \mathcal{Y} \mathcal{D}$ as a composition of morphisms in ${ }_{H}^{H} \mathcal{Y D}$.

Let $n \in \mathbb{N}_{0}$, let $C^{4}=Q \otimes Q \otimes Q \otimes Q$ and let $u \in C_{(n)}^{4}=\sum_{i+j+k+l \leq n} Q_{i} \otimes Q_{j} \otimes Q_{k} \otimes Q_{l}$.

A direct computation rewriting the cocycle condition using (21) proves that, for every $n \in \mathbb{N}_{0}$, and $u \in C_{(n)}^{4}$

$$
\sum_{0 \leq i+j \leq n}\left[\omega_{i}(Q \otimes Q \otimes m) * \omega_{j}(m \otimes Q \otimes Q)\right](u)
$$




$$
=\sum_{0 \leq a+b+c \leq n}\left[\left(\varepsilon \otimes \omega_{a}\right) * \omega_{b}(Q \otimes m \otimes Q) *\left(\omega_{c} \otimes \varepsilon\right)\right](u) .
$$

Next aim is to check that $\left[\bar{\omega}_{s}\right] \in \mathrm{H}_{\mathcal{Y D}}^{3}(\operatorname{gr} Q, \mathbb{k})$ i.e. that

$$
\bar{\omega}_{s}\left(m_{\bar{Q}} \otimes \bar{Q} \otimes \bar{Q}\right)+\bar{\omega}_{s}\left(\bar{Q} \otimes \bar{Q} \otimes m_{\bar{Q}}\right)=\left(\varepsilon_{\bar{Q}} \otimes \bar{\omega}_{s}\right)+\bar{\omega}_{s}\left(\bar{Q} \otimes m_{\bar{Q}} \otimes \bar{Q}\right)+\left(\bar{\omega}_{s} \otimes \varepsilon_{\bar{Q}}\right) .
$$

This is achieved by evaluating the two sides of the equality above on $\bar{u}:=\bar{x} \otimes \bar{y} \otimes \bar{z} \otimes \bar{t}$ where $x, y, z, t$ are elements in the basis and using (20). If $\bar{u}$ has homogeneous degree greater than $s$, then both terms are zero. Otherwise, i.e. if $\bar{u}$ has homogeneous degree at most $s$, one has $\bar{\omega}_{s}\left(m_{\bar{Q}} \otimes \bar{Q} \otimes \bar{Q}\right)(\bar{u})=\omega_{s}\left(m_{Q} \otimes Q \otimes Q\right)(u)$ and similarly for the other pieces so that one has to check that

$$
\omega_{s}(m \otimes Q \otimes Q)(u)+\omega_{s}(Q \otimes Q \otimes m)(u)=\left(\varepsilon \otimes \omega_{s}\right)(u)+\omega_{s}(Q \otimes m \otimes Q)(u)+\left(\omega_{s} \otimes \varepsilon\right)(u) .
$$

This equality follows by using $(23)$ and the definition of $s$. that

By assumption $\mathrm{H}_{\mathcal{Y} \mathcal{D}}^{3}(\operatorname{gr} Q, \mathbb{k})=0$ so that there exists a morphism $\bar{v}: \bar{Q} \otimes \bar{Q} \rightarrow \mathbb{k}$ in ${ }_{H}^{H} \mathcal{Y D}$ such

Explicitly, on elements in the basis we get

$$
\bar{\omega}_{s}=\partial^{2} \bar{v}=\bar{v} \otimes \varepsilon_{\bar{Q}}-\bar{v}\left(\bar{Q} \otimes m_{\bar{Q}}\right)+\bar{v}\left(m_{\bar{Q}} \otimes \bar{Q}\right)-\varepsilon_{\bar{Q}} \otimes \bar{v}
$$

$$
\bar{\omega}_{s}(\bar{x} \otimes \bar{y} \otimes \bar{z})=\bar{v}(\bar{x} \otimes \bar{y}) \varepsilon_{\bar{Q}}(\bar{z})-\bar{v}(\bar{x} \otimes \bar{y} \cdot \bar{z})+\bar{v}(\bar{x} \cdot \bar{y} \otimes \bar{z})-\varepsilon_{\bar{Q}}(\bar{x}) \bar{v}(\bar{y} \otimes \bar{z}) .
$$

Define $\bar{\zeta}: \bar{Q} \otimes \bar{Q} \rightarrow \mathbb{k}$ on the basis by setting

$$
\bar{\zeta}(\bar{x} \otimes \bar{y}):=\delta_{|x|+|y|, s} \bar{v}(\bar{x} \otimes \bar{y}) .
$$

As we have done for $\omega_{t}$, one can check that $\bar{\zeta}$ is a morphism in ${ }_{H}^{H} \mathcal{Y} \mathcal{D}$.

Moreover on elements in the basis we get

$$
\begin{aligned}
& \left(\partial^{2} \bar{\zeta}\right)(\bar{x} \otimes \bar{y} \otimes \bar{z}) \\
= & \left(\bar{\zeta} \otimes \varepsilon_{\bar{Q}}\right)(\bar{x} \otimes \bar{y} \otimes \bar{z})-\bar{\zeta}\left(\bar{Q} \otimes m_{\bar{Q}}\right)(\bar{x} \otimes \bar{y} \otimes \bar{z})+\bar{\zeta}\left(m_{\bar{Q}} \otimes \bar{Q}\right)(\bar{x} \otimes \bar{y} \otimes \bar{z})-\left(\varepsilon_{\bar{Q}} \otimes \bar{\zeta}\right)(\bar{x} \otimes \bar{y} \otimes \bar{z}) \\
= & \bar{\zeta}(\bar{x} \otimes \bar{y}) \varepsilon_{\bar{Q}}(\bar{z})-\bar{\zeta}(\bar{x} \otimes \bar{y} \cdot \bar{z})+\bar{\zeta}(\bar{x} \cdot \bar{y} \otimes \bar{z})-\varepsilon_{\bar{Q}}(\bar{x}) \bar{\zeta}(\bar{y} \otimes \bar{z}) .
\end{aligned}
$$

By using (20), one gets

$$
\bar{\zeta}(\bar{x} \otimes \bar{y} \cdot \bar{z})=\delta_{|x|+|y|+|z|, s} \bar{v}(\bar{x} \otimes \bar{y} \cdot \bar{z}) \quad \text { and } \quad \bar{\zeta}(\bar{x} \cdot \bar{y} \otimes \bar{z})=\delta_{|x|+|y|+|z|, s} \bar{v}(\bar{x} \cdot \bar{y} \otimes \bar{z}) .
$$

By means of these equalities one gets

$$
\begin{aligned}
\left(\partial^{2} \bar{\zeta}\right)(\bar{x} \otimes \bar{y} \otimes \bar{z}) & =\delta_{|x|+|y|+|z|, s}\left(\partial^{2} \bar{v}\right)(\bar{x} \otimes \bar{y} \otimes \bar{z})=\delta_{|x|+|y|+|z|, s} \bar{\omega}_{s}(\bar{x} \otimes \bar{y} \otimes \bar{z}) \\
& =\delta_{|x|+|y|+|z|, s} \omega_{s}(x \otimes y \otimes z)=\delta_{|x|+|y|+|z|, s} \delta_{|x|+|y|+|z|, s} \omega(x \otimes y \otimes z) \\
& =\delta_{|x|+|y|+|z|, s} \omega(x \otimes y \otimes z)=\omega_{s}(x \otimes y \otimes z)=\bar{\omega}_{s}(\bar{x} \otimes \bar{y} \otimes \bar{z}) .
\end{aligned}
$$

Therefore $\partial^{2} \bar{\zeta}=\bar{\omega}_{s}$. This means that we can assume that $\bar{v}(\bar{x} \otimes \bar{y})=0$ for $|x|+|y| \neq s$. Equivalently

$$
\bar{v}(\bar{x} \otimes \bar{y})=\delta_{|x|+|y|, s} \bar{v}(\bar{x} \otimes \bar{y}) \text { for } x, y \text { in the basis. }
$$

Set

In particular, one gets

$$
v(x \otimes y)=\delta_{|x|+|y|, s} v(x \otimes y) \text { for } x, y \text { in the basis. }
$$

Note also that both $v$ and $\gamma$ are morphisms in ${ }_{H}^{H} \mathcal{Y D}$ as they are obtained as composition or sum of morphisms in this category. Let us check that $\gamma$ is a gauge transformation on $Q$ in ${ }_{H}^{H} \mathcal{Y D}$.

Recall that $x^{0,0}=1_{Q}$ is in the basis. For $x$ in the basis, we have $\gamma\left(x \otimes 1_{Q}\right)=\varepsilon(x)+v\left(x \otimes 1_{Q}\right)$. Note that

$$
\begin{aligned}
0 & =\delta_{|x|, s} \varepsilon(x)=\delta_{|x|+\left|1_{Q}\right|+\left|1_{Q}\right|, s} \omega\left(x \otimes 1_{Q} \otimes 1_{Q}\right) \\
& =\omega_{s}\left(x \otimes 1_{Q} \otimes 1_{Q}\right)=\bar{\omega}_{s}\left(\bar{x} \otimes \overline{1_{Q}} \otimes \overline{1_{Q}}\right)
\end{aligned}
$$




$$
\begin{array}{ll}
= & \bar{v}\left(\bar{x} \otimes \overline{1_{Q}}\right) \varepsilon_{\bar{Q}}\left(\overline{1_{Q}}\right)-\bar{v}\left(\bar{x} \otimes \overline{1_{Q}} \cdot \overline{1_{Q}}\right)+\bar{v}\left(\bar{x} \cdot \overline{1_{Q}} \otimes \overline{1_{Q}}\right)-\varepsilon_{\bar{Q}}(\bar{x}) \bar{v}\left(\overline{1_{Q}} \otimes \overline{1_{Q}}\right) \\
& \bar{v}\left(\bar{x} \otimes \overline{1_{Q}}\right)-\bar{v}\left(\bar{x} \otimes \overline{1_{Q}}\right)+\bar{v}\left(\bar{x} \otimes \overline{1_{Q}}\right)-\varepsilon_{\bar{Q}}(\bar{x}) \delta_{\left|1_{Q}\right|+\left|1_{Q}\right|, s} \bar{v}\left(\overline{1_{Q}} \otimes \overline{1_{Q}}\right) \\
= & v\left(x \otimes 1_{Q}\right)
\end{array}
$$

so that $v\left(x \otimes 1_{Q}\right)=0$ and hence $\gamma\left(x \otimes 1_{Q}\right)=\varepsilon(x)+v\left(x \otimes 1_{Q}\right)=\varepsilon(x)$. Similarly one proves $\gamma\left(1_{Q} \otimes x\right)=\varepsilon(x)$. Hence $\gamma$ is unital. Note that the coalgebra $C=Q \otimes Q$ is connected as $Q$ is. Thus, in order to prove that $\gamma: Q \otimes Q \rightarrow \mathbb{k}$ is convolution invertible it suffices to check (see $\mathbb{M d}$, Lemma 5.2.10]) that $\gamma_{\mid \mathbb{k} 1_{Q} \otimes \mathbb{k} 1_{Q}}$ is convolution invertible. But for $k, k^{\prime} \in \mathbb{k}$ we have

$$
\gamma\left(k 1_{Q} \otimes k^{\prime} 1_{Q}\right)=k k^{\prime} \gamma\left(1_{Q} \otimes 1_{Q}\right)=k k^{\prime} \varepsilon\left(1_{Q}\right)=k k^{\prime}=(\varepsilon \otimes \varepsilon)\left(k 1_{Q} \otimes k^{\prime} 1_{Q}\right)
$$

Hence $\gamma_{\mid \mathbb{k} 1_{Q} \otimes \mathbb{k} 1_{Q}}=(\varepsilon \otimes \varepsilon)_{\mid \mathbb{k} 1_{Q} \otimes \mathbb{k} 1_{Q}}$ which is convolution invertible. Thus there is a $\mathbb{k}$-linear map $\gamma^{-1}: Q \otimes Q \rightarrow \mathbb{k}$ and such that

$$
\gamma * \gamma^{-1}=\varepsilon \otimes \varepsilon=\gamma^{-1} * \gamma
$$

Note that, by Lemma 2.3, $\gamma \in{ }_{H}^{H} \mathcal{Y} \mathcal{D}$ implies $\gamma^{-1} \in{ }_{H}^{H} \mathcal{Y} \mathcal{D}$.

Therefore $\gamma$ is a gauge transformation in ${ }_{H}^{H} \mathcal{Y} \mathcal{D}$. By Proposition 2.4, $Q^{\gamma}$ is a coquasi-bialgebra in ${ }_{H}^{H} \mathcal{Y} \mathcal{D}$. By Proposition 2.6, we have that $\operatorname{gr} Q^{\gamma}$ and $\operatorname{gr} Q$ coincide as bialgebras in ${ }_{H}^{H} \mathcal{Y} \mathcal{D}$. Hence $\mathrm{H}_{\mathcal{Y} \mathcal{D}}^{3}\left(\operatorname{gr} Q^{\gamma}, \mathbb{k}\right)=\mathrm{H}_{\mathcal{Y} \mathcal{D}}^{3}(\operatorname{gr} Q, \mathbb{k})=0$. Therefore $Q^{\gamma}$ fulfills the same requirement of $Q$ as in the statement. Let us check that $\left(\omega^{\gamma}\right)_{t}=0$ for $1 \leq t \leq s$ (this will complete the proof by an induction process as $Q$ is finite-dimensional).

Note that the definition of $\gamma$ and (25) imply

$$
\gamma(x \otimes y)=\delta_{|x|+|y|, 0} \gamma(x \otimes y)+\delta_{|x|+|y|, s} \gamma(x \otimes y) \text { for } x, y \text { in the basis. }
$$

Let $C^{2}=Q \otimes Q$ and let $C_{(n)}^{2}=\sum_{i+j \leq n} Q_{i} \otimes Q_{j}$. For $u \in C_{(2 s-1)}^{2}$ we have

$$
[\gamma *((\varepsilon \otimes \varepsilon)-v)](u)=(\varepsilon \otimes \varepsilon)(u)-v(u)+v(u)-v\left(u_{1}\right) v\left(u_{2}\right) \stackrel{25}{=}(\varepsilon \otimes \varepsilon)(u) .
$$

Therefore $[\gamma *((\varepsilon \otimes \varepsilon)-v)]_{\mid C_{(2 s-1)}^{2}}=(\varepsilon \otimes \varepsilon)_{\mid C_{(2 s-1)}^{2}}$. By uniqueness of the convolution inverse, we deduce

$$
\gamma^{-1}(u)=(\varepsilon \otimes \varepsilon)(u)-v(u), \text { for } u \in C_{(2 s-1)}^{2} .
$$

Let $x, y, z$ be in the basis. Set $\bar{u}:=\bar{x} \otimes \bar{y} \otimes \bar{z}$ and $u:=x \otimes y \otimes z$. We compute

$$
\begin{aligned}
\left(\omega^{\gamma}\right)_{s}(u) & =\delta_{|x|+|y|+|z|, s} \omega^{\gamma}(u) \\
& =\delta_{|x|+|y|+|z|, s}\left[(\varepsilon \otimes \gamma) * \gamma(Q \otimes m) * \omega * \gamma^{-1}(m \otimes Q) *\left(\gamma^{-1} \otimes \varepsilon\right)\right](u) \\
& =\delta_{|x|+|y|+|z|, s}\left[(\varepsilon \otimes \gamma) * \gamma(Q \otimes m) *\left(\omega_{0}+\omega_{s}\right) * \gamma^{-1}(m \otimes Q) *\left(\gamma^{-1} \otimes \varepsilon\right)\right](u) \\
& \\
& \delta_{|x|+|y|+|z|, s}\left[\begin{array}{c}
(\varepsilon \otimes \gamma) * \gamma(Q \otimes m) * \gamma^{-1}(m \otimes Q) *\left(\gamma^{-1} \otimes \varepsilon\right)+ \\
(\varepsilon \otimes \gamma) * \gamma(Q \otimes m) * \omega_{s} * \gamma^{-1}(m \otimes Q) *\left(\gamma^{-1} \otimes \varepsilon\right)
\end{array}\right](u) \\
= & {\left[\begin{array}{c}
\delta_{|x|+|y|+|z|, s}(\varepsilon \otimes \gamma)\left(u_{1}\right) \cdot \gamma(Q \otimes m)\left(u_{2}\right) \cdot \gamma^{-1}(m \otimes Q)\left(u_{3}\right) \cdot\left(\gamma^{-1} \otimes \varepsilon\right)\left(u_{4}\right)+ \\
\delta_{|x|+|y|+|z|, s}(\varepsilon \otimes \gamma)\left(u_{1}\right) \cdot \gamma(Q \otimes m)\left(u_{2}\right) \cdot \omega_{s}\left(u_{3}\right) \cdot \gamma^{-1}(m \otimes Q)\left(u_{4}\right) \cdot\left(\gamma^{-1} \otimes \varepsilon\right)\left(u_{5}\right)
\end{array}\right] . }
\end{aligned}
$$

Now, all terms appearing in the last two lines, excepted $\omega_{s}$, vanish out of degrees 0 and $s$ and coincide with $\varepsilon \otimes \varepsilon \otimes \varepsilon$ on degree 0 . On the other hand $\omega_{s}$ vanishes out of $s$. Since $\gamma:=(\varepsilon \otimes \varepsilon)+v$ and in view of (27), the term $\delta_{|x|+|y|+|z|, s}$ forces the following simplification

$$
\left(\omega^{\gamma}\right)_{s}(u)=\left[\begin{array}{c}
\delta_{|x|+|y|+|z|, s}[(\varepsilon \otimes v)(u)+v(Q \otimes m)(u)-v(m \otimes Q)(u)-(v \otimes \varepsilon)(u)]+ \\
+\delta_{|x|+|y|+|z|, s} \omega_{s}(u)
\end{array}\right] .
$$

Now $\omega_{s}(u)=\bar{\omega}_{s}(\bar{u})$ while one proves that $(\varepsilon \otimes v)(u)=\left(\varepsilon_{\bar{Q}} \otimes \bar{v}\right)(\bar{u}), \delta_{|x|+|y|+|z|, s} v(m \otimes Q)(u)=$ $\delta_{|x|+|y|+|z|, s} \bar{v}\left(m_{\bar{Q}} \otimes \bar{Q}\right)(\bar{u})$ and similarly for the other pieces of the equality.

Thus one gets

$$
\left(\omega^{\gamma}\right)_{s}(u)=\left[\begin{array}{c}
\delta_{|x|+|y|+|z|, s}\left[\left(\varepsilon_{\bar{Q}} \otimes \bar{v}\right)(\bar{u})+\bar{v}\left(\bar{Q} \otimes m_{\bar{Q}}\right)(\bar{u})-\bar{v}\left(m_{\bar{Q}} \otimes \bar{Q}\right)(\bar{u})-\left(\bar{v} \otimes \varepsilon_{\bar{Q}}\right)(\bar{u})\right]+ \\
+\delta_{|x|+|y|+|z|, s} \bar{\omega}_{s}(\bar{u})
\end{array}\right]
$$




$$
=\quad-\delta_{|x|+|y|+|z|, s} \partial^{2} \bar{v}+\delta_{|x|+|y|+|z|, s} \bar{\omega}_{s}(\bar{u})=0 .
$$

For $0 \leq t \leq s-1$, analogously to the above, we compute

$$
\begin{aligned}
\left(\omega^{\gamma}\right)_{t}(u)= & \delta_{|x|+|y|+|z|, t} \omega^{\gamma}(u) \\
= & \delta_{|x|+|y|+|z|, t}\left[(\varepsilon \otimes \gamma) * \gamma(Q \otimes m) * \omega * \gamma^{-1}(m \otimes Q) *\left(\gamma^{-1} \otimes \varepsilon\right)\right](u) \\
= & \delta_{|x|+|y|+|z|, t}\left[(\varepsilon \otimes \gamma) * \gamma(Q \otimes m) * \omega_{0} * \gamma^{-1}(m \otimes Q) *\left(\gamma^{-1} \otimes \varepsilon\right)\right](u) \\
\text { 22 } & \delta_{|x|+|y|+|z|, t}\left[(\varepsilon \otimes \gamma) * \gamma(Q \otimes m) * \gamma^{-1}(m \otimes Q) *\left(\gamma^{-1} \otimes \varepsilon\right)\right](u) \\
= & \delta_{|x|+|y|+|z|, t}(\varepsilon \otimes \varepsilon \otimes \varepsilon)(u)=\delta_{0, t}(\varepsilon \otimes \varepsilon \otimes \varepsilon)(u) .
\end{aligned}
$$

Therefore we can now repeat the argument on $\omega^{\gamma}$ instead of $\omega$. Deforming several times we will get a reassociator, say $\omega^{\prime}$, whose first non trivial component $\omega_{t}^{\prime}$, with $t \neq 0$, exceeds the dimension of $Q$. In other words $\omega^{\prime}=\omega_{0}^{\prime}$ which is trivial. Hence $Q$ is gauge equivalent to a connected bialgebra in ${ }_{H}^{H} \mathcal{Y D}$.

\section{INVARIANTS}

Given a $\mathbb{k}$-algebra $A$, we denote by $\mathrm{H}^{n}(A,-)$ the $n$-th right derived functor of $\operatorname{Hom}_{A, A}(A,-)$ in the category of $A$-bimodules. In other words, for every $A$-bimodule $M, \mathrm{H}^{n}(A, M)$ is the Hochschild cohomology group of $A$ with coefficients in $M$. Denote by $\mathrm{Z}^{n}(A, M)$ and $\mathrm{B}^{n}(A, M)$ the abelian groups of $n$-cocycles and of $n$-coboundaries respectively.

Let $H$ be a Hopf algebra, let $B$ be a left $H$-module algebra and let $M$ be a $B \# H$-bimodule, where $B \# H$ denotes the smash product algebra, see e.g. [Mo, Definition 4.1.3]. Then $\mathrm{H}^{n}(B, M)$ becomes an $H$-bimodule as follows. Its structure of left $H$-module is given via $\varepsilon_{H}$ and its structure of right $H$-module is defined, for every $f \in \mathrm{Z}^{n}(B, M)$ and $h \in H$, by setting

$$
[f] h:=\left[\chi_{n}^{h}(M)(f)\right]
$$

where, for every $k \in \mathbb{k}, b_{1}, \ldots, b_{n} \in B$, we set

$$
\begin{array}{rll}
\chi_{0}^{h}(M)(f)(k):= & \left(1_{B} \# S\left(h_{1}\right)\right) f(k)\left(1_{B} \# h_{2}\right) \text { for } n=0 \text { while and for } n \geq 1 \\
\chi_{n}^{h}(M)(f)\left(b_{1} \otimes b_{2} \otimes \cdots \otimes b_{n}\right):= & \left(1_{B} \# S\left(h_{1}\right)\right) f\left(h_{2} b_{1} \otimes h_{3} b_{2} \otimes \cdots \otimes h_{n+1} b_{n}\right)\left(1_{B} \# h_{n+2}\right) .
\end{array}
$$

Moreover

$$
\partial^{n} \circ \chi_{n}^{h}(M)=\chi_{n+1}^{h}(M) \circ \partial^{n}, \text { for every } n \geq-1,
$$

where $\partial^{n}: \operatorname{Hom}_{\mathbb{k}}\left(B^{\otimes n}, M\right) \rightarrow \operatorname{Hom}_{\mathbb{k}}\left(B^{\otimes(n+1)}, M\right)$ denotes the differential of the usual Hochschild cohomology.

Denote by $\mathrm{H}^{n}(B, M)^{H}$ the space of $H$-invariant elements of $\mathrm{H}^{n}(B, M)$.

Proposition 4.1. Let $H$ be a semisimple Hopf algebra and let $B$ be a left $H$-module algebra. Denote by $A:=B \# H$. Then, for each $n \in \mathbb{N}_{0}$ and for every A-bimodule $M$

$$
\mathrm{H}^{n}(B \# H, M) \cong \mathrm{H}^{n}(B, M)^{H} .
$$

Proof. We will apply Stt, Equation (3.6.1)]. To this aim we have to prove first that $A / B$ is an $H$-Galois extension such that $A$ is flat as left and right $B$-module. Now, $A=B \#_{\xi} H$ for $\xi: H \otimes H \rightarrow B$ defined by $\xi(x, y)=\varepsilon_{H}(x) \varepsilon_{H}(y) 1_{A}$, cf. [Mo, Definition 7.1.1]. Moreover a direct computation shows that $\iota: B \rightarrow A: b \mapsto b \# 1_{H}$ is a right $H$-extension where $A$ is regarded as a right $H$-comodule via $\rho: A \rightarrow A \otimes H: b \# h \mapsto\left(b \# h_{1}\right) \otimes h_{2}$. Thus, by [Mo, Proposition 7.2.7], we know that $\iota: B \rightarrow A$ is $H$-cleft and hence, by [Mo, Theorem 8.2.4], it is $H$-Galois. The $B$-bimodule structure of $A$ is induced by $\iota$ so that, explicitly, we have

$$
\begin{aligned}
& b^{\prime}(b \# h)=\left(b^{\prime} \# 1_{H}\right)(b \# h)=b^{\prime} b \# h, \\
& (b \# h) b^{\prime}=(b \# h)\left(b^{\prime} \# 1_{H}\right)=b\left(h_{1} b^{\prime}\right) \# h_{2} .
\end{aligned}
$$


Note that $A=B \# H$ is flat as a left $B$-module as $H$ is a free $\mathbb{k}$-module ( $\mathbb{k}$ is a field). Now consider the map $\alpha: H \otimes B \rightarrow A$ defined by setting $\alpha(h \otimes b):=h_{1} b \otimes h_{2}$ (note that it is defined as the braiding in $\left.{ }_{H}^{H} \mathcal{Y} \mathcal{D}\right)$. We have

$$
\alpha\left(h \otimes b b^{\prime}\right)=h_{1}\left(b b^{\prime}\right) \otimes h_{2}=\left(h_{1} b\right)\left(h_{2} b^{\prime}\right) \otimes h_{3}=\left(h_{1} b \# h_{2}\right) b^{\prime}=\alpha(h \otimes b) b^{\prime}
$$

so that $\alpha$ is right $B$-linear where $H \otimes B$ is regarded as a right module via $(h \# b) b^{\prime}:=h \# b b^{\prime}$. Now $H$ is semisimple and hence separable (see Stt, Corollary 3.7]). Thus $H$ is finite-dimensional and hence it has bijective antipode $S_{H}$. Thus $\alpha$ is invertible with inverse given by $\alpha^{-1}(b \# h):=h_{2} \otimes S_{H}^{-1}\left(h_{1}\right) b$. Therefore $\alpha$ is an isomorphism of right $B$-modules and hence $A$ is flat as a right $B$-module as $H \otimes B$ is.

We have now the hypotheses necessary to apply [Stf, Equation (3.6.1)] and obtain

$$
\mathrm{H}^{n}(A, M) \cong \operatorname{Hom}_{-, H}\left(\mathbb{k}, \mathrm{H}^{n}(B, M)\right)=\operatorname{Hom}_{\mathbb{k}}\left(\mathbb{k}, \mathrm{H}^{n}(B, M)\right)^{H} \cong \mathrm{H}^{n}(B, M)^{H} .
$$

Remark 4.2. Proposition 4.1 in the particular case when $M=\mathbb{k}$ and $B$ is finite-dimensional is [SV, Theorem 2.17]. Note that in the notations therein, one has $E(B)=\oplus_{n \in \mathbb{N}_{0}} E_{n}(B, \mathbb{k})$ where $E_{n}(B, \mathbb{k})=\operatorname{Ext}_{B}^{n}(\mathbb{k}, \mathbb{k}) \cong \mathrm{H}^{n}(B, \mathbb{k})$. The latter isomorphism is [CE. Corollary 4.4, page 170].

Let $H$ be a Hopf algebra and let $B$ be a bialgebra in the braided category ${ }_{H}^{H} \mathcal{Y D}$. Denote by $A:=B \# H$ the Radford-Majid bosonization of $B$ by $H$, see e.g. Ra3, Theorem 1]. Note that $A$ is endowed with an algebra map $\varepsilon_{A}: A \rightarrow \mathbb{k}$ defined by $\varepsilon_{A}(b \# h)=\varepsilon_{B}(b) \varepsilon_{H}(h)$ so that we can regard $\mathbb{k}$ as an $A$-bimodule via $\varepsilon_{A}$. Then we can consider $\mathrm{H}^{n}(B, \mathbb{k})$ as an $H$-bimodule as follows. Its structure of left $H$-module is given via $\varepsilon_{H}$ and its structure of right $H$-module is defined, for every $f \in \mathrm{Z}^{n}(B, \mathbb{k})$ and $h \in H$, by setting

$$
[f] h:=[f h],
$$

where $(f h)(z)=f(h z)$, for every $z \in B^{\otimes n}$. The latter is the usual right $H$-module structure of $\operatorname{Hom}_{\mathbb{k}}\left(B^{\otimes n}, \mathbb{k}\right)$. Indeed, for every $n \geq-1$, the vector space $\operatorname{Hom}_{\mathbb{k}}\left(B^{\otimes n}, \mathbb{k}\right)$ is an $H$-bimodule with respect to this right $H$-module structure and the left one induced by $\varepsilon_{H}$.

Corollary 4.3. Let $H$ be a semisimple Hopf algebra and let $B$ be a bialgebra in the braided category ${ }_{H}^{H} \mathcal{Y} \mathcal{D}$. Set $A:=B \# H$. Then, for each $n \in \mathbb{N}_{0}$

$$
\mathrm{H}^{n}(B \# H, \mathbb{k}) \cong \mathrm{H}^{n}(B, \mathbb{k})^{H}
$$

and the differential $\partial^{n}: \operatorname{Hom}_{\mathbb{k}}\left(B^{\otimes n}, \mathbb{k}\right) \rightarrow \operatorname{Hom}_{\mathbb{k}}\left(B^{\otimes(n+1)}, \mathbb{k}\right)$ of the usual Hochschild cohomology is $H$-bilinear.

Proof. In the particular case $M=\mathbb{k}$, the right module $H$-structure used in Proposition 4.1 simplifies as follows. It is defined, for every $f \in \mathrm{Z}^{n}(B, \mathbb{k})$ and $h \in H$, by setting

$$
[f] h:=\left[\chi_{n}^{h}(\mathbb{k})(f)\right]
$$

where, for every $k \in \mathbb{k}, b_{1}, \ldots, b_{n} \in B$, we set

$$
\begin{aligned}
\chi_{0}^{h}(\mathbb{k})(f)(k) & :=\varepsilon_{H}(h) f(k) \text { for } n=0 \text { while and for } n \geq 1 \\
\chi_{n}^{h}(\mathbb{k})(f)\left(b_{1} \otimes b_{2} \otimes \cdots \otimes b_{n}\right) & :=f\left(h_{1} b_{1} \otimes h_{2} b_{2} \otimes \cdots \otimes h_{n} b_{n}\right) .
\end{aligned}
$$

More concisely $\chi_{n}^{h}(\mathbb{k})(f)(z)=f(h z)$ for every $n \in \mathbb{N}_{0}$ and $z \in B^{\otimes n}$ i.e. $[f] h:=[f h]$ where $f h:=\chi_{n}^{h}(\mathbb{k})(f)$.

Now consider the differential $\partial^{n}: \operatorname{Hom}_{\mathbb{k}}\left(B^{\otimes n}, \mathbb{k}\right) \rightarrow \operatorname{Hom}_{\mathbb{k}}\left(B^{\otimes(n+1)}, \mathbb{k}\right)$ of the usual Hochschild cohomology. Note that for each $n \in \mathbb{N}_{0}, \operatorname{Hom}_{\mathbb{k}}\left(B^{\otimes n}, \mathbb{k}\right)$ is regarded as a bimodule over $H$ using the left $H$-module structures of its arguments. By (28), we have

$$
\partial^{n} \chi_{n}^{h}(\mathbb{k})(f)=\chi_{n+1}^{h}(\mathbb{k}) \partial^{n}(f)
$$

Since $\chi_{n}^{h}(\mathbb{k})(f)=f h$, the last displayed equality becomes $\partial^{n}(f h)=\partial^{n}(f) h$ for every $n \in \mathbb{N}_{0}$. Thus $\partial^{n}$ is right $H$-linear. Since $h f=\varepsilon_{H}(h) f$ for every $f \in \operatorname{Hom}_{\mathbb{k}}\left(B^{\otimes n}, \mathbb{k}\right), h \in H$, we get that $\partial^{n}$ is also left $H$-linear whence $H$-bilinear. 
Remark 4.4. Note that, in the context of the proof of [EG, Proposition 5.1], one has

$$
\mathrm{H}^{3}\left(\mathcal{B}(V) \# \mathbb{C}\left[\mathbb{Z}_{p}\right], \mathbb{C}\right) \cong \mathrm{H}^{3}(\mathcal{B}(V), \mathbb{C})^{\mathbb{Z}_{p}} .
$$

This is a particular case of Corollary 4.3 where $H=\mathbb{C}\left[\mathbb{Z}_{p}\right], V \in{ }_{H}^{H} \mathcal{Y D}$ and $B=\mathcal{B}(V)$.

Proposition 4.5. Let $\mathcal{C}$ and $\mathcal{D}$ be abelian categories. Let $r, \omega: \mathcal{C} \rightarrow \mathcal{D}$ be exact functors such that $r$ is a subfunctor of $\omega$ i.e. there is a natural transformation $\eta: r \rightarrow \omega$ which is a monomorphism when evaluated on objects. If $X$ is a subobject of $Y$ then $r(X)=\omega(X) \cap r(Y)$. Moreover, for every morphism $f: X \rightarrow Y$ in $\mathcal{C}$ one has

$$
\begin{aligned}
\operatorname{ker}(r(f)) & =r(\operatorname{ker}(f))=\omega(\operatorname{ker}(f)) \cap r(X)=\operatorname{ker}(\omega(f)) \cap r(X), \\
\operatorname{Im}(r(f)) & =\operatorname{Im}(\omega(f)) \cap r(Y)=r(\operatorname{Im}(f)) .
\end{aligned}
$$

Proof. The proof is similar to $\mathrm{Stn}$, Proposition 1.7, page 138].

Remark 4.6. From Corollary 4.3, we have

$$
\begin{aligned}
\mathrm{H}^{n}(B, \mathbb{k})^{H} & =\left\{[f] \mid f \in \mathrm{Z}^{n}(B, \mathbb{k}), \varepsilon_{H}(h)[f]=[f] h, \text { for every } h \in H\right\} \\
& =\left\{[f] \mid f \in \mathrm{Z}^{n}(B, \mathbb{k}),\left[\varepsilon_{H}(h) f\right]=[f h], \text { for every } h \in H\right\}
\end{aligned}
$$

where, for every $z \in B^{\otimes n}$, we have

$$
(f h)(z)=f(h z) .
$$

Note that, for any $H$-bimodule $M$ one has

$$
\operatorname{Hom}_{H, H}(H, M) \cong M^{H}=\{m \in M \mid h m=m h \text {, for every } h \in H\} .
$$

Note also that $H$ is a separable $\mathbb{k}$-algebra whence it is projective in the category of $H$-bimodules. As a consequence $\operatorname{Hom}_{H, H}(H,-) \cong(-)^{H}:{ }_{H} \mathfrak{M}_{H} \rightarrow \mathfrak{M}$ is an exact functor (here ${ }_{H} \mathfrak{M}_{H}$ is the category of $H$-bimodules and $\mathfrak{M}$ the category of $\mathbb{k}$-vector spaces). By Proposition 1.5 applied to the case when $r:=(-)^{H}:{ }_{H} \mathfrak{M}_{H} \rightarrow \mathfrak{M}$ and $\omega$ is the forgetful functor, for every morphism $f: X \rightarrow Y$ of $H$-bimodules one has

$$
\operatorname{ker}\left(f^{H}\right)=\operatorname{ker}(f) \cap X^{H}=(\operatorname{ker}(f))^{H} \quad \text { and } \quad \operatorname{Im}\left(f^{H}\right)=\operatorname{Im}(f) \cap Y^{H}=(\operatorname{Im}(f))^{H} .
$$

Still by Corollary 4.3 , we know that the differential $\partial^{n}: \operatorname{Hom}_{\mathbb{k}}\left(B^{\otimes n}, \mathbb{k}\right) \longrightarrow \operatorname{Hom}_{\mathbb{k}}\left(B^{\otimes(n+1)}, \mathbb{k}\right)$ of the usual Hochschild cohomology is $H$-bilinear. Thus we can apply the argument above to get

$$
\begin{aligned}
\operatorname{ker}\left(\left(\partial^{n}\right)^{H}\right) & =\operatorname{ker}\left(\partial^{n}\right) \cap \operatorname{Hom}_{\mathbb{k}}\left(B^{\otimes n}, \mathbb{k}\right)^{H}=\left(\operatorname{ker}\left(\partial^{n}\right)\right)^{H} \quad \text { and } \\
\operatorname{Im}\left(\left(\partial^{n-1}\right)^{H}\right) & =\operatorname{Im}\left(\partial^{n-1}\right) \cap \operatorname{Hom}_{\mathbb{k}}\left(B^{\otimes n}, \mathbb{k}\right)^{H}=\left(\operatorname{Im}\left(\partial^{n-1}\right)\right)^{H} .
\end{aligned}
$$

Now $\operatorname{Hom}_{\mathbb{k}}\left(B^{\otimes n}, \mathbb{k}\right)^{H}=\operatorname{Hom}_{H,-}\left(B^{\otimes n}, \mathbb{k}\right)$ so that we get

$$
\begin{aligned}
& \mathrm{Z}_{H-\text { Mod }}^{n}(B, \mathbb{k})=\mathrm{Z}^{n}(B, \mathbb{k}) \cap \operatorname{Hom}_{H,-}\left(B^{\otimes n}, \mathbb{k}\right)=\mathrm{Z}^{n}(B, \mathbb{k})^{H} \quad \text { and } \\
& \mathrm{B}_{H-\text { Mod }}^{n}(B, \mathbb{k})=\mathrm{B}^{n}(B, \mathbb{k}) \cap \operatorname{Hom}_{H,-}\left(B^{\otimes n}, \mathbb{k}\right)=\mathrm{B}^{n}(B, \mathbb{k})^{H} .
\end{aligned}
$$

where $\mathrm{Z}_{H \text {-Mod }}^{n}(B, \mathbb{k})$ and $\mathrm{B}_{H \text {-Mod }}^{n}(B, \mathbb{k})$ denotes the the abelian groups of $n$-cocycles, of $n$-coboundaries for the cohomology of the algebra $B$ with coefficients in $\mathbb{k}$ computed in the monoidal category $H$ Mod of left $H$-modules. The corresponding $n$-th Hochschild cohomology group is

$$
\mathrm{H}_{H-\text { Mod }}^{n}(B, \mathbb{k}):=\frac{\mathrm{Z}_{H-\text { Mod }}^{n}(B, \mathbb{k})}{\mathrm{B}_{H-\text { Mod }}^{n}(B, \mathbb{k})}=\frac{\mathrm{Z}^{n}(B, \mathbb{k})^{H}}{\mathrm{~B}^{n}(B, \mathbb{k})^{H}} \cong\left(\frac{\mathrm{Z}^{n}(B, \mathbb{k})}{\mathrm{B}^{n}(B, \mathbb{k})}\right)^{H}=\mathrm{H}^{n}(B, \mathbb{k})^{H} .
$$

Denote by $D(H)$ the Drinfeld double, see e.g. the first structure of Maj, Theorem 7.1.1].

Proposition 4.7. In the setting of Corollary 4.9 assume that $H$ is also cosemisimple. Then, for $n \in \mathbb{N}_{0}$

$\mathrm{Z}_{\mathcal{Y} \mathcal{D}}^{n}(B, \mathbb{k})=\mathrm{Z}^{n}(B, \mathbb{k})^{D(H)}, \quad \mathrm{B}_{\mathcal{Y} \mathcal{D}}^{n}(B, \mathbb{k})=\mathrm{B}^{n}(B, \mathbb{k})^{D(H)} \quad$ and $\quad \mathrm{H}_{\mathcal{Y} \mathcal{D}}^{n}(B, \mathbb{k}) \cong \mathrm{H}^{n}(B, \mathbb{k})^{D(H)}$. where $\mathrm{Z}^{n}(B, \mathbb{k})$ and $\mathrm{B}^{n}(B, \mathbb{k})$ are regarded as $D(H)$-subbimodules of $\operatorname{Hom}_{\mathbb{k}}\left(B^{\otimes n}, \mathbb{k}\right)$ whose structure is induced by the left $D(H)$-module structures of its arguments. 
Moreover $\mathrm{H}^{n}(B, \mathbb{k})^{D(H)}$ is a subspace of $\mathrm{H}^{n}(B, \mathbb{k})^{H}$.

Proof. For shortness, in this proof, we denote $D(H)$ by $D$. Consider the analogue of the standard complex as in Remark 3.1

$$
{ }_{H}^{H} \mathcal{Y D}(\mathbb{k}, \mathbb{k}) \stackrel{\partial^{0}}{\longrightarrow}{ }_{H}^{H} \mathcal{Y} \mathcal{D}(B, \mathbb{k}) \stackrel{\partial^{1}}{\longrightarrow}{ }_{H}^{H} \mathcal{Y} \mathcal{D}\left(B^{\otimes 2}, \mathbb{k}\right) \stackrel{\partial^{2}}{\longrightarrow} \cdots
$$

where $\partial^{n}$ is induced by the differential $\partial^{n}: \operatorname{Hom}_{\mathbb{k}}\left(B^{\otimes n}, \mathbb{k}\right) \longrightarrow \operatorname{Hom}_{\mathbb{k}}\left(B^{\otimes(n+1)}, \mathbb{k}\right)$ of the ordinary Hochschild cohomology. Now, since $H$ is semisimple, it is finite-dimensional (whence it has bijective antipode) so that, by a result essentially due to Majid (see [Mo, Proposition 10.6.16]) and by [RT, Proposition 6], we get a category isomorphism ${ }_{H}^{H} \mathcal{Y D} \cong{ }_{D} \mathfrak{M}$. Thus the complex above can be rewritten as follows

$$
\operatorname{Hom}_{D,-}(\mathbb{k}, \mathbb{k}) \stackrel{\partial^{0}}{\longrightarrow} \operatorname{Hom}_{D,-}(B, \mathbb{k}) \stackrel{\partial^{1}}{\longrightarrow} \operatorname{Hom}_{D,-}\left(B^{\otimes 2}, \mathbb{k}\right) \stackrel{\partial^{2}}{\longrightarrow} \cdots
$$

Now, since, for each $n \in \mathbb{N}_{0}$, we have $\operatorname{Hom}_{D,-}\left(B^{\otimes n}, \mathbb{k}\right)=\operatorname{Hom}_{\mathbb{k}}\left(B^{\otimes n}, \mathbb{k}\right)^{D}$, we obtain the complex

$$
\operatorname{Hom}_{\mathbb{k}}(\mathbb{k}, \mathbb{k})^{D} \stackrel{\partial^{0}}{\longrightarrow} \operatorname{Hom}_{\mathbb{k}}(B, \mathbb{k})^{D} \stackrel{\partial^{1}}{\longrightarrow} \operatorname{Hom}_{\mathbb{k}}\left(B^{\otimes 2}, \mathbb{k}\right)^{D} \stackrel{\partial^{2}}{\longrightarrow} \cdots
$$

We will write $\left(\partial^{n}\right)^{D}$ instead of $\partial^{n}$ when we would like to stress that the map considered is the one induced on invariants. Thus we will write equivalently

$$
\operatorname{Hom}_{\mathbb{k}}(\mathbb{k}, \mathbb{k})^{D} \stackrel{\left(\partial^{0}\right)^{D}}{\longrightarrow} \operatorname{Hom}_{\mathbb{k}}(B, \mathbb{k})^{D} \stackrel{\left(\partial^{1}\right)^{D}}{\longrightarrow} \operatorname{Hom}_{\mathbb{k}}\left(B^{\otimes 2}, \mathbb{k}\right)^{D} \stackrel{\left(\partial^{2}\right)^{D}}{\longrightarrow} \cdots
$$

Now, assume $H$ is also cosemisimple. Since $H$ is both semisimple and cosemisimple, by Ra2, Proposition 7] the Hopf algebra $D$ is semisimple as an algebra. Thus, as in Remark 4.6 in case of $H$, the functor $(-)^{D}:{ }_{D} \mathfrak{M}_{D} \rightarrow \mathfrak{M}$ is exact (here ${ }_{D} \mathfrak{M}_{D}$ is the category of $D$-bimodules and $\mathfrak{M}$ the category of $\mathbb{k}$-vector spaces). By Proposition 4.5 applied to the case when $r:=(-)^{D}:{ }_{D} \mathfrak{M}_{D} \rightarrow \mathfrak{M}$ and $\omega$ is the forgetful functor, for every morphism $f: X \rightarrow Y$ of $D$-bimodules one has

$$
\operatorname{ker}\left(f^{D}\right)=\operatorname{ker}(f) \cap X^{D}=(\operatorname{ker}(f))^{D} \quad \text { and } \quad \operatorname{Im}\left(f^{D}\right)=\operatorname{Im}(f) \cap Y^{D}=(\operatorname{Im}(f))^{D} .
$$

In particular we get

$$
\begin{aligned}
\operatorname{ker}\left(\left(\partial^{n}\right)^{D}\right) & =\operatorname{ker}\left(\partial^{n}\right) \cap \operatorname{Hom}_{\mathbb{k}}\left(B^{\otimes n}, \mathbb{k}\right)^{D}=\operatorname{ker}\left(\partial^{n}\right)^{D} \quad \text { and } \\
\operatorname{Im}\left(\left(\partial^{n-1}\right)^{D}\right) & =\operatorname{Im}\left(\partial^{n-1}\right) \cap \operatorname{Hom}_{\mathbb{k}}\left(B^{\otimes n}, \mathbb{k}\right)^{D}=\operatorname{Im}\left(\partial^{n-1}\right)^{D}
\end{aligned}
$$

and hence

$$
\begin{aligned}
& \mathrm{Z}_{\mathcal{Y} \mathcal{D}}^{n}(B, \mathbb{k})=\mathrm{Z}^{n}(B, \mathbb{k}) \cap \operatorname{Hom}_{D,-}\left(B^{\otimes n}, \mathbb{k}\right)=\mathrm{Z}^{n}(B, \mathbb{k})^{D} \quad \text { and } \\
& \mathrm{B}_{\mathcal{Y} \mathcal{D}}^{n}(B, \mathbb{k})=\mathrm{B}^{n}(B, \mathbb{k}) \cap \operatorname{Hom}_{D,-}\left(B^{\otimes n}, \mathbb{k}\right)=\mathrm{B}^{n}(B, \mathbb{k})^{D}
\end{aligned}
$$

Then we obtain

$$
\mathrm{H}_{\mathcal{Y} \mathcal{D}}^{n}(B, \mathbb{k})=\frac{\mathrm{Z}_{\mathcal{Y} \mathcal{D}}^{n}(B, \mathbb{k})}{\mathrm{B}_{\mathcal{Y} \mathcal{D}}^{n}(B, \mathbb{k})}=\frac{\mathrm{Z}^{n}(B, \mathbb{k})^{D}}{\mathrm{~B}^{n}(B, \mathbb{k})^{D}} \cong \mathrm{H}^{n}(B, \mathbb{k})^{D} .
$$

Let us prove the last part of the statement. The correspondence between the left $D$-module structure and the structure of Yetter-Drinfeld module over $H$ is written explicitly in Maj, Proposition 7.1.6]. In particular $D=H^{*} \otimes H$ and given $V \in{ }_{H}^{H} \mathcal{Y D}$, the two structures are related by the following equality $(f \otimes h) \triangleright v=f\left((h \triangleright v)_{-1}\right)(h \triangleright v)_{0}$ for every $f \in H^{*}, h \in H, v \in V$. Thus $\left(\varepsilon_{H} \otimes h\right) \triangleright v=h \triangleright v$. Moreover $H$ is a Hopf subalgebra of $D$ via $h \mapsto \varepsilon_{H} \otimes h$, where $D$ is considered with the first structure of Maj, Theorem 7.1.1]. Since the $D$-bimodule structure of $\mathrm{H}^{n}(B, \mathbb{k})$ is induced by the one of $\operatorname{Hom}_{\mathbb{k}}\left(B^{\otimes n}, \mathbb{k}\right)$ which comes from the left $D$-module structures of its arguments and similarly for the $H$-bimodule structure of $\mathrm{H}^{n}(B, \mathbb{k})$, we deduce that $\mathrm{H}^{n}(B, \mathbb{k})^{D}$ is a subspace of $\mathrm{H}^{n}(B, \mathbb{k})^{H}$. 
EXAMPLE 4.8. In the setting of the proof of An, Theorem 4.1.3], a Nichols algebra $\mathcal{B}(V)$ such that $\mathrm{H}^{3}(\mathcal{B}(V), \mathbb{k})^{\mathbb{Z}_{m}}=0$ is considered where $\mathbb{k}$ is a field of characteristic zero. By Proposition 4.7 applied in the case $H=\mathbb{k}_{m}$ and $B=\mathcal{B}(V)$, we have that $\mathrm{H}_{\mathcal{Y} \mathcal{D}}^{3}(\mathcal{B}(V), \mathbb{k}) \cong \mathrm{H}^{3}(\mathcal{B}(V), \mathbb{k})^{D(H)}$ is a subspace of $\mathrm{H}^{3}(\mathcal{B}(V), \mathbb{k})^{H}=\mathrm{H}^{3}(\mathcal{B}(V), \mathbb{k})^{\mathbb{Z}_{m}}=0$. Thus we get $\mathrm{H}_{\mathcal{Y} \mathcal{D}}^{3}(\mathcal{B}(V), \mathbb{k})=0$. Therefore, in view of Theorem 3.2, if $(Q, m, u, \Delta, \varepsilon, \omega)$ is a f.d. connected coquasi-bialgebra in ${ }_{H}^{H} \mathcal{Y} \mathcal{D}$ such that $\operatorname{gr} Q \cong \mathcal{B}(V)$ (as above) as augmented algebras in ${ }_{H}^{H} \mathcal{Y D}$ (the counit must be the same in order to have the same Yetter-Drinfeld module structure on $\mathbb{k}$ ), then we can conclude that $Q$ is gauge equivalent to a connected bialgebra in ${ }_{H}^{H} \mathcal{Y} \mathcal{D}$.

Remark 4.9. Let $A$ be a finite-dimensional coquasi-bialgebra with the dual Chevalley property i.e. the coradical $H$ of $A$ is a coquasi-subbialgebra of $A$ (in particular $H$ is cosemisimple). Assume the coquasi-bialgebra structure of $H$ has trivial reassociator (i.e. it is an ordinary bialgebra) and also assume it has an antipode (i.e. it is a Hopf algebra). Then, by [AP, Corollary 6.4], $\operatorname{gr} A$ is isomorphic to $R \# H$ as a coquasi-bialgebra, where $R$ is a suitable connected bialgebra in ${ }_{H}^{H} \mathcal{Y} \mathcal{D}$. Note that $R \# H$ is the usual Radford-Majid bosonization as $H$ has trivial reassociator, see AP, Definition 5.4]. Hence we can compute

$$
\mathrm{H}^{3}(\operatorname{gr} A, \mathbb{k})=\mathrm{H}^{3}(R \# H, \mathbb{k}) .
$$

Assume further that $H$ is semisimple. Then, by Corollary 1.3, we have

$$
\mathrm{H}^{n}(R \# H, \mathbb{k}) \cong \mathrm{H}^{n}(R, \mathbb{k})^{H}
$$

so that $\mathrm{H}^{3}(\operatorname{gr} A, \mathbb{k}) \cong \mathrm{H}^{3}(R, \mathbb{k})^{H}$. Thus, if $\mathrm{H}^{3}(R, \mathbb{k})^{H}=0$, one gets $\mathrm{H}^{3}(\operatorname{gr} A, \mathbb{k})=0$ which is the analogue of the condition EG. Proposition 2.3] (note that our $A$ is the dual of the one considered therein) which guarantees that $A$ is gauge equivalent to an ordinary Hopf algebra, if $A$ has an a quasi-antipode and $\mathbb{k}=\mathbb{C}$. Next we will give another approach to arrive at the same conclusion but just requiring $\mathrm{H}_{\mathcal{Y} \mathcal{D}}^{3}(R, \mathbb{k})=0$. Note that a priori $\mathrm{H}_{\mathcal{Y} \mathcal{D}}^{3}(R, \mathbb{k}) \cong \mathrm{H}^{3}(R, \mathbb{k})^{D(H)}$ is smaller than $\mathrm{H}^{3}(R, \mathbb{k})^{H}$.

\section{Dual Chevalley}

The main aim of this section is to prove Theorem 5.6. Let $A$ be a Hopf algebra over a field $\mathbb{k}$ of characteristic zero such that the coradical $H$ of $A$ is a sub-Hopf algebra (i.e. $A$ has the dual Chevalley Property). Assume $H$ is finite-dimensional so that $H$ is semisimple. By $\mathrm{ABM}$, Theorem I], there is a gauge transformation $\zeta: A \otimes A \rightarrow \mathbb{k}$ such that $A^{\zeta}$ is isomorphic, as a coquasi-bialgebra, to the bosonization $Q \# H$ of a connected coquasi-bialgebra $Q$ in ${ }_{H}^{H} \mathcal{Y D}$ by $H$. By construction $\zeta$ is $H$-bilinear and $H$-balanced: this follows from ABM, Proposition 5.7] (note that gauge transformation $v_{B}: B \otimes B \rightarrow \mathbb{k}$, used therein for $B:=R \#_{\xi} H$, is $H$-bilinear and $H$-balanced, as observed in the proof) and the fact that there is an $H$-bilinear Hopf algebra isomorphism $\psi: B \rightarrow A$ (see ABM Proof of Theorem I, page 36 and Theorem 6.1] which is a consequence of AMS1, Theorem 3.64]) where $(R, \xi)$ is a suitable connected pre-bialgebra with cocycle in ${ }_{H}^{H} \mathcal{Y D}$ (note that $\zeta=v_{B} \circ\left(\psi^{-1} \otimes \psi^{-1}\right)$ ): here by connected pre-bialgebra we mean that the coradical $R_{0}$ of $R$ is $\mathbb{k}_{R}$ (by the properties of $1_{R}$ this implies that $R_{0}$ is a subcoalgebra in ${ }_{H}^{H} \mathcal{Y D}$ of $R$ ). Assume that $A$ is finite-dimensional. Then $Q \# H$ and hence $Q$ is finite dimensional.

Thus, by Theorem 3.2, if $\mathrm{H}_{\mathcal{Y} \mathcal{D}}^{3}(\operatorname{gr} Q, \mathbb{k})=0$, then $Q$ is gauge equivalent to a connected bialgebra in ${ }_{H}^{H} \mathcal{Y D}$.

First let us check which condition on $A$ guarantee that $\mathrm{H}_{\mathcal{Y D}}^{3}(\operatorname{gr} Q, \mathbb{k})=0$. Note that by construction $Q=R^{v}$ (see $\mathrm{ABM}$, Proposition 5.7]) where $v:=(\lambda \xi)^{-1}$, the convolution inverse of $\lambda \xi$ and $\lambda: H \rightarrow \mathbb{k}$ denotes the total integral on $H$. Thus we can rewrite $\operatorname{gr}(Q)$ as $\operatorname{gr}\left(R^{v}\right)$.

Moreover $v_{B}$ is given by $v_{B}\left((r \# h) \otimes\left(r^{\prime} \# h^{\prime}\right)\right)=v\left(r \otimes h r^{\prime}\right) \varepsilon_{H}\left(h^{\prime}\right)$ for every $r, r^{\prime} \in R, h, h^{\prime} \in H$. By AMStu, Proposition 2.5], gr $(R)$ inherits the pre-bialgebra structure in ${ }_{H}^{H} \mathcal{Y D}$ of $R$. This is proved by checking that $R_{i} \cdot R_{j} \subseteq R_{i+j}$ for every $i, j \in \mathbb{N}_{0}$, where $R_{i}$ denotes the $i$-th term of the coradical filtration of $R$. Moreover $R_{i}$ is a subcoalgebra of $R$ in ${ }_{H}^{H} \mathcal{Y} \mathcal{D}$. 
LEMmA 5.1. Keep the above hypotheses and notations. Then $\operatorname{gr}\left(R^{v}\right)$ and $\operatorname{gr}(R)$ coincide as bialgebras in ${ }_{H}^{H} \mathcal{Y D}$ where the structures of $\operatorname{gr}(R)$ are induced by the ones of $(R, \xi)$.

Proof. By Theorem 1.5, gr $\left(R^{v}\right)=\operatorname{gr}(Q)$ is a connected bialgebras in ${ }_{H}^{H} \mathcal{Y} \mathcal{D}$.

Note that $R^{v}$ and $R$ coincide as coalgebras in ${ }_{H}^{H} \mathcal{Y} \mathcal{D}$ so that $\operatorname{gr}\left(R^{v}\right)$ and $\operatorname{gr}(R)$ coincide as coalgebras in ${ }_{H}^{H} \mathcal{Y} \mathcal{D}$. They also have the same unit. It remains to check that their two multiplications coincide too.

Since $\xi$ is unital, by [AMS1, Proposition 4.8], we have that $v$ is unital and this is equivalent to $v^{-1}$ unital (see the proof therein).

Let $C:=R \otimes R$. Let $n>0$ and let $w \in C_{(n)}=\sum_{i+j \leq n} R_{i} \otimes R_{j}$. By [AMS1, Lemma 3.69], we have that

Thus we get

$$
\Delta_{C}(w)-w \otimes\left(1_{R}\right)^{\otimes 2}-\left(1_{R}\right)^{\otimes 2} \otimes w \in C_{(n-1)} \otimes C_{(n-1)} .
$$

$$
w_{1} \otimes w_{2} \otimes w_{3}-\Delta_{C}(w) \otimes\left(1_{R}\right)^{\otimes 2}-\Delta_{C}\left(\left(1_{R}\right)^{\otimes 2}\right) \otimes w \in \Delta_{C}\left(C_{(n-1)}\right) \otimes C_{(n-1)}
$$

and hence

$w_{1} \otimes w_{2} \otimes w_{3}-w \otimes\left(1_{R}\right)^{\otimes 2} \otimes\left(1_{R}\right)^{\otimes 2}-\left(1_{R}\right)^{\otimes 2} \otimes w \otimes\left(1_{R}\right)^{\otimes 2}-\left(1_{R}\right)^{\otimes 4} \otimes w \in C_{(n-1)} \otimes C_{(n-1)} \otimes C_{(n-1)}$.

Since $m\left(C_{(n-1)}\right) \subseteq \sum_{i+j \leq n} m\left(R_{i} \otimes R_{j}\right) \subseteq R_{n-1}$ we get

$w_{1} \otimes m\left(w_{2}\right) \otimes w_{3}-w \otimes 1_{R} \otimes\left(1_{R}\right)^{\otimes 2}-\left(1_{R}\right)^{\otimes 2} \otimes m(w) \otimes\left(1_{R}\right)^{\otimes 2}-\left(1_{R}\right)^{\otimes 3} \otimes w \in C_{(n-1)} \otimes R_{n-1} \otimes C_{(n-1)}$

and hence

$$
w_{1} \otimes\left(m\left(w_{2}\right)+R_{n-1}\right) \otimes w_{3}=\left(1_{R}\right)^{\otimes 2} \otimes\left(m(w)+R_{n-1}\right) \otimes\left(1_{R}\right)^{\otimes 2} .
$$

Let $x, y \in R$. We compute

$$
\begin{aligned}
\bar{x} \cdot v \bar{y} & =\left(x+R_{|x|-1}\right) \cdot v\left(y+R_{|y|-1}\right) \\
& =(x \cdot v y)+R_{|x|+|y|-1}=m^{v}(x \otimes y)+R_{|x|+|y|-1} \\
& =v\left((x \otimes y)_{1}\right) m\left((x \otimes y)_{2}\right) v^{-1}\left((x \otimes y)_{3}\right)+R_{|x|+|y|-1} \\
& =v\left((x \otimes y)_{1}\right)\left(m\left((x \otimes y)_{2}\right)+R_{|x|+|y|-1}\right) v^{-1}\left((x \otimes y)_{3}\right) \\
& v\left(\left(1_{R}\right)^{\otimes 2}\right)\left(m(x \otimes y)+R_{|x|+|y|-1}\right) v^{-1}\left(\left(1_{R}\right)^{\otimes 2}\right) \\
& =m(x \otimes y)+R_{|x|+|y|-1}=(x \cdot y)+R_{|x|+|y|-1}=\bar{x} \cdot \bar{y} .
\end{aligned}
$$

The following result is inspired by AMS1, Theorem 3.71].

LEMma 5.2. Let $H$ be a cosemisimple Hopf algebra. Let $C$ be a left $H$-comodule coalgebra such that $C_{0}$ is a one-dimensional left $H$-comodule subcoalgebra of $C$. Let $B=C \# H$ be the smash coproduct of $C$ by $H$ i.e. the coalgebra defined by

$$
\begin{aligned}
\Delta_{B}(c \# h) & =\sum\left(c_{1} \#\left(c_{2}\right)_{\langle-1\rangle} h_{1}\right) \otimes\left(\left(c_{2}\right)_{\langle 0\rangle} \# h_{2}\right), \\
\varepsilon_{B}(c \# h) & =\varepsilon_{C}(c) \varepsilon_{H}(h) .
\end{aligned}
$$

Then, for every $n \in \mathbb{N}_{0}$ we have $B_{n}=C_{n} \# H$.

Proof. Since $C_{0}$ is a subcoalgebra of $C$ in ${ }^{H} \mathfrak{M}$ and, for $n \geq 1$, one has $C_{n}=C_{n-1} \wedge_{C} C_{0}$, then inductively one proves that $C_{n}$ is a subcoalgebra of $C$ in ${ }^{H} \mathfrak{M}$. Set $B_{(n)}:=C_{n} \# H$ for every $n \in \mathbb{N}_{0}$. Let us check that $B_{(n)}=B_{n}$ by induction on $n \in \mathbb{N}_{0}$.

Let $n=0$. First note $B=\cup_{m \in \mathbb{N}_{0}} B_{(m)}$ and, since $\Delta_{C}\left(C_{m}\right) \subseteq \sum_{0 \leq i \leq m} C_{i} \otimes C_{m-i}$, we also have

$$
\begin{aligned}
\Delta_{B}\left(B_{(m)}\right) & =\Delta_{B}\left(C_{m} \# H\right) \subseteq \sum_{0 \leq i \leq m} \sum\left(C_{i} \#\left(C_{m-i}\right)_{\langle-1\rangle}(H)_{1}\right) \otimes\left(\left(C_{m-i}\right)_{\langle 0\rangle} \#(H)_{2}\right) \\
& \subseteq \sum_{0 \leq i \leq m}\left(C_{i} \# H\right) \otimes\left(C_{m-i} \#(H)\right)=\sum_{0 \leq i \leq m} B_{(i)} \otimes B_{(m-i)} .
\end{aligned}
$$


Therefore $\left(B_{(m)}\right)_{m \in \mathbb{N}_{0}}$ is a coalgebra filtration for $B$ and hence, by [Sw, Proposition 11.1.1], we get that $B_{(0)} \supseteq B_{0}$. Since $C_{0}$ is one-dimensional, there is a grouplike element $1_{C} \in C_{0}$ such that $C_{0}=\mathbb{k} 1_{C}$. Moreover one checks that $C_{0}$ is a subcoalgebra of $C$ in ${ }^{H} \mathfrak{M}$ implies $\sum\left(1_{C}\right)_{\langle-1\rangle} \otimes$ $\left(1_{C}\right)_{\langle 0\rangle}=1_{H} \otimes 1_{C}$.

Let $\sigma: H \rightarrow C \otimes H: h \mapsto 1_{C} \otimes h$ be the canonical injection. We have

$$
\begin{aligned}
\Delta_{B} \sigma(h) & =\Delta_{B}\left(1_{C} \otimes h\right)=\sum\left(1_{C} \#\left(1_{C}\right)_{\langle-1\rangle} h_{1}\right) \otimes\left(\left(1_{C}\right)_{\langle 0\rangle} \# h_{2}\right) \\
& =\sum\left(1_{C} \# 1_{H} h_{1}\right) \otimes\left(1_{C} \# h_{2}\right)=\sum \sigma\left(h_{1}\right) \otimes \sigma\left(h_{2}\right)=(\sigma \otimes \sigma) \Delta_{H}(h), \\
\varepsilon_{B} \sigma(h) & =\varepsilon_{B}\left(1_{C} \otimes h\right)=\varepsilon_{C}\left(1_{C}\right) \varepsilon_{H}(h)=\varepsilon_{H}(h)
\end{aligned}
$$

so that $\sigma$ is a coalgebra map. Since $H$ is cosemisimple and $\sigma$ an injective coalgebra map we deduce that also $\sigma(H)=C_{0} \otimes H=B_{(0)}$ is a cosemisimple subcoalgebra of $B$ whence $B_{(0)} \subseteq B_{0}$.

Let $n>0$ and assume that $B_{i}=B_{(i)}$ for $0 \leq i \leq n-1$. Let $\sum_{i \in I} c_{i} \# h_{i} \in B_{n}$. Then

$$
\Delta_{B}\left(\sum_{i \in I} c_{i} \# h_{i}\right) \in B_{n-1} \otimes B+B \otimes B_{0}=C_{n-1} \otimes H \otimes C \otimes H+C \otimes H \otimes C_{0} \otimes H .
$$

Let $p_{n}: C \rightarrow \frac{C}{C_{n}}$ be the canonical projection. If we apply $\left(p_{n-1} \otimes \varepsilon_{H} \otimes p_{0} \otimes H\right)$ we get

$$
\begin{aligned}
0 & =\left(p_{n-1} \otimes \varepsilon_{H} \otimes p_{0} \otimes H\right) \Delta_{B}\left(\sum_{i \in I} c_{i} \# h_{i}\right) \\
& =\left(p_{n-1} \otimes \varepsilon_{H} \otimes p_{0} \otimes H\right)\left(\sum_{i \in I}\left(\left(c_{i}\right)_{1} \#\left(\left(c_{i}\right)_{2}\right)_{\langle-1\rangle}\left(h_{i}\right)_{1}\right) \otimes\left(\left(\left(c_{i}\right)_{2}\right)_{\langle 0\rangle} \#\left(h_{i}\right)_{2}\right)\right) \\
& =\left(p_{n-1} \otimes p_{0} \otimes H\right)\left(\sum_{i \in I}\left(c_{i}\right)_{1} \otimes\left(c_{i}\right)_{2} \otimes h_{i}\right)=\left(\left(p_{n-1} \otimes p_{0}\right) \Delta_{C} \otimes H\right)\left(\sum_{i \in I} c_{i} \# h_{i}\right) .
\end{aligned}
$$

Thus $\sum_{i \in I} c_{i} \# h_{i} \in \operatorname{ker}\left(\left(p_{n-1} \otimes p_{0}\right) \Delta_{C} \otimes H\right)=\left[\operatorname{ker}\left(\left(p_{n-1} \otimes p_{0}\right) \Delta_{C}\right)\right] \otimes H=C_{n} \otimes H=B_{(n)}$. Thus $B_{n} \subseteq B_{(n)}$. On the other hand, form $\Delta_{C}\left(C_{n}\right) \subseteq C_{n-1} \otimes C+C \otimes C_{0}$ we deduce

$$
\begin{aligned}
\Delta_{B}\left(B_{(n)}\right) & =\Delta_{B}\left(C_{n} \otimes H\right) \\
& \subseteq \sum\left(\left(C_{n}\right)_{1} \#\left(\left(C_{n}\right)_{2}\right)_{\langle-1\rangle}(H)_{1}\right) \otimes\left(\left(\left(C_{n}\right)_{2}\right)_{\langle 0\rangle} \#(H)_{2}\right) \\
& \subseteq \sum\left(C_{n-1} \#(C)_{\langle-1\rangle} H\right) \otimes\left((C)_{\langle 0\rangle} \# H\right)+\sum\left(C \#\left(C_{0}\right)_{\langle-1\rangle} H\right) \otimes\left(\left(C_{0}\right)_{\langle 0\rangle} \# H\right) \\
& \subseteq\left(C_{n-1} \# H\right) \otimes(C \# H)+(C \# H) \otimes\left(C_{0} \# H\right) \\
& =B_{(n-1)} \otimes B+B \otimes B_{(0)}=B_{n-1} \otimes B+B \otimes B_{0}
\end{aligned}
$$

and hence $B_{(n)} \subseteq B_{n}$.

Definition 5.3. Let $A$ be a Hopf algebra over a field $\mathbb{k}$ such that the coradical $H$ of $A$ is a subHopf algebra (i.e. $A$ has the dual Chevalley Property). Set $G:=\operatorname{gr}(A)$. There are two canonical Hopf algebra maps

$$
\begin{aligned}
\sigma_{G}: & H \rightarrow \operatorname{gr}(A): h \mapsto h+A_{-1}, \\
\pi_{G}: & \operatorname{gr}(A) \rightarrow H: a+A_{n-1} \mapsto a \delta_{n, 0}, \quad n \in \mathbb{N}_{0} .
\end{aligned}
$$

The diagram of $A$ (see AS1, page 659]) is the vector space

$$
\mathcal{D}(A):=\left\{d \in \operatorname{gr}(A) \mid \sum d_{1} \otimes \pi_{G}\left(d_{2}\right)=d \otimes 1_{H}\right\} .
$$

It is a bialgebra in ${ }_{H}^{H} \mathcal{Y D}$ as follows. $\mathcal{D}(A)$ is a subalgebra of $G$. The left $H$-action, the left $H$-coaction of $\mathcal{D}(A)$, the comultiplication and counit are given respectively by

$$
h \triangleright d:=\sum \sigma_{G}\left(h_{1}\right) d \sigma_{G} S\left(h_{2}\right), \quad \rho(d)=\sum \pi_{G}\left(d_{1}\right) \otimes d_{2},
$$




$$
\Delta_{D(A)}(d):=\sum d_{1} \sigma_{G} S_{H} \pi_{G}\left(d_{2}\right) \otimes d_{3}, \quad \varepsilon_{D(A)}(d)=\varepsilon_{G}(d) .
$$

Although the following result seems to be folklore, we include here its statement for future references.

Proposition 5.4. Let $A$ be a Hopf algebra over a field $\mathbb{k}$ such that the coradical $H$ of $A$ is a sub-Hopf algebra. Let $A^{\prime}$ be a Hopf algebra over a field $\mathbb{k}$. Let $f: A^{\prime} \rightarrow A$ be an isomorphism of Hopf algebras. Then $H^{\prime}:=f^{-1}(H) \cong H$ is the coradical of $A^{\prime}$ and it is a sub-Hopf algebra of $A^{\prime}$. Thus we can identify $H^{\prime}$ with $H$. Moreover $f$ induces an isomorphism $\mathcal{D}(f): \mathcal{D}\left(A^{\prime}\right) \rightarrow \mathcal{D}(A)$ of bialgebras in ${ }_{H}^{H} \mathcal{Y D}$.

Proposition 5.5. Keep the hypotheses and notations of the beginning of the section. Then $\mathcal{D}(A) \cong$ $\mathcal{D}\left(R \#_{\xi} H\right) \cong \operatorname{gr}(R)$ as bialgebras in ${ }_{H}^{H} \mathcal{Y D}$.

Proof. Apply Proposition 5.4 to the canonical isomorphism $\psi: B:=R \#{ }_{\xi} H \rightarrow A$ that we recalled at the beginning of the section to get that $\mathcal{D}\left(R \#_{\xi} H\right) \cong \mathcal{D}(A)$. Note that, by $H$-linearity we have

$$
\psi\left(1_{R} \# h\right)=\psi\left(\left(1_{R} \# 1_{H}\right)\left(1_{R} \# h\right)\right)=\psi\left(\left(1_{R} \# 1_{H}\right) h\right)=\psi\left(1_{R} \# 1_{H}\right) h=h
$$

so that $\psi\left(\mathbb{k} 1_{R} \otimes H\right)=H$ and hence $H^{\prime}=\psi^{-1}(H)=\mathbb{k} 1_{R} \otimes H$ with the notation of Proposition 5.4. Thus $B_{0}=\mathbb{k} 1_{R} \otimes H=R_{0} \otimes H$ so that we can identify $B_{0}$ with $H$ via the canonical isomorphism $H \rightarrow R_{0} \otimes H: h \mapsto 1_{R} \otimes h$. Its inverse is $R_{0} \otimes H \rightarrow H: r \otimes h \mapsto \varepsilon_{R}(r) h$. With this identification and by setting $G:=\operatorname{gr}(B)$, we can consider the canonical bialgebra maps

$$
\begin{aligned}
\sigma_{G}: & H \rightarrow \operatorname{gr}(B): h \mapsto 1_{R} \# h+\left(R \#_{\xi} H\right)_{-1}, \\
\pi_{G}: & \operatorname{gr}(B) \rightarrow H: r \# h+(R \# \xi H)_{n-1} \mapsto \varepsilon_{R}(r) h \delta_{n, 0}, \text { where } r \# h \in(R \# \xi H)_{n}, n \in \mathbb{N}_{0} .
\end{aligned}
$$

Since the underlying coalgebra of $B$ is exactly the smash coproduct of $R$ by $H$ and $(R, \xi)$ is a connected pre-bialgebra with cocycle in ${ }_{H}^{H} \mathcal{Y D}$, by Lemma 5.2, we have that $B_{n}=R_{n} \otimes H$. Let us compute $\mathcal{D}:=\mathcal{D}(B)$. As a vector space it is

$$
\mathcal{D}:=\left\{d \in G \mid \sum d_{1} \otimes \pi_{G}\left(d_{2}\right)=d \otimes 1_{H}\right\} .
$$

By [AS1, Lemma 2.1], we have that $\mathcal{D}=\oplus_{n \in \mathbb{N}_{0}} \mathcal{D}^{n}$ where $\mathcal{D}^{n}=\mathcal{D} \cap G^{n}=\mathcal{D} \cap \frac{B_{n}}{B_{n-1}}$. Let $d:=$ $\overline{\sum_{i \in I} r_{i} \# h_{i}} \in \mathcal{D}^{n}$ where we can assume $\sum_{i \in I} r_{i} \# h_{i} \in B_{n} \backslash B_{n-1}$ and, for every $i \in I, r_{i} \# h_{i} \in B_{n} \backslash B_{n-1}$. Then $\overline{\sum_{i \in I} r_{i} \# h_{i}}=\sum_{i \in I} \overline{r_{i} \# h_{i}}$ and hence the fact that $d$ is coinvariant rewrites as

$$
\sum_{i \in I}\left(\overline{r_{i} \# h_{i}}\right)_{1} \otimes \pi_{G}\left(\left(\overline{r_{i} \# h_{i}}\right)_{2}\right)=\sum_{i \in I} \overline{r_{i} \# h_{i}} \otimes 1_{H} .
$$

By definition of $\pi_{G}$ and (1), the left-hand side becomes

$$
\sum_{i \in I}\left(\overline{r_{i} \# h_{i}}\right)_{1} \otimes \pi_{G}\left(\left(\overline{r_{i} \# h_{i}}\right)_{2}\right)=\sum_{i \in I}\left(\left(r_{i} \#\left(h_{i}\right)_{1}\right)+B_{n-1}\right) \otimes\left(h_{i}\right)_{2}
$$

so that (31) becomes

$$
\sum_{i \in I}\left(\left(r_{i} \#\left(h_{i}\right)_{1}\right)+B_{n-1}\right) \otimes\left(h_{i}\right)_{2}=\sum_{i \in I} \overline{r_{i} \# h_{i}} \otimes 1_{H}=\sum_{i \in I}\left(r_{i} \# h_{i}+B_{n-1}\right) \otimes 1_{H}
$$

i.e.

$$
\sum_{i \in I}\left(r_{i} \#\left(h_{i}\right)_{1}\right) \otimes\left(h_{i}\right)_{2}-\sum_{i \in I} r_{i} \# h_{i} \otimes 1_{H} \in B_{n-1} \otimes H=R_{n-1} \otimes H \otimes H .
$$

If we apply $R \otimes \varepsilon_{H} \otimes H$, we get

$$
\sum_{i \in I} r_{i} \otimes h_{i}-\sum_{i \in I} r_{i} \varepsilon_{H}\left(h_{i}\right) \otimes 1_{H} \in R_{n-1} \otimes H=B_{n-1} .
$$

Thus $\overline{\sum_{i \in I} r_{i} \# h_{i}}=\sum_{i \in I} \overline{r_{i} \# h_{i}}=\sum_{i \in I}\left(r_{i} \# h_{i}+B_{n-1}\right)=\sum_{i \in I}\left(r_{i} \varepsilon_{H}\left(h_{i}\right) \otimes 1_{H}\right)+B_{n-1}$. 
Since $\sum_{i \in I} r_{i} \# h_{i} \in B_{n} \backslash B_{n-1}$ we get that $\left(\sum_{i \in I} r_{i} \varepsilon_{H}\left(h_{i}\right)\right) \otimes 1_{H} \notin B_{n-1}$ and hence $\sum_{i \in I} r_{i} \varepsilon_{H}\left(h_{i}\right) \notin$ $R_{n-1}$ and we can write

$$
\overline{\sum_{i \in I} r_{i} \# h_{i}}=\overline{\left(\sum_{i \in I} r_{i} \varepsilon_{H}\left(h_{i}\right)\right) \otimes 1_{H}} .
$$

Therefore we have proved that the map

$$
\varphi_{n}: \frac{R_{n}}{R_{n-1}} \rightarrow \mathcal{D}^{n}: \bar{r} \mapsto \overline{r \otimes 1_{H}},
$$

which is well-defined as $\mathcal{D}^{n}=\mathcal{D} \cap G^{n}=\mathcal{D} \cap \frac{B_{n}}{B_{n-1}}=\mathcal{D} \cap \frac{R_{n} \otimes H}{R_{n-1} \otimes H}$, is also surjective.

It is also injective as $\varphi_{n}(\bar{r})=\varphi_{n}(\bar{s})$ implies $r \otimes 1_{H}-s \otimes 1_{H} \in B_{n-1}=R_{n-1} \otimes H$ and hence, by applying $R \otimes \varepsilon_{H}$, we get $r-s \in R_{n-1}$ i.e. $\bar{r}=\bar{s}$. Therefore $\varphi_{n}$ is an isomorphism such that $\overline{\sum_{i \in I} r_{i} \# h_{i}}=\varphi_{n}\left(\overline{\sum_{i \in I} r_{i} \varepsilon_{H}\left(h_{i}\right)}\right)$ and hence

$$
\varphi_{n}^{-1}\left(\overline{\sum_{i \in I} r_{i} \# h_{i}}\right)=\overline{\sum_{i \in I} r_{i} \varepsilon_{H}\left(h_{i}\right)} .
$$

Clearly this extends to a graded $\mathbb{k}$-linear isomorphism

$$
\varphi: \operatorname{gr}(R) \rightarrow \mathcal{D} \text {. }
$$

Let us check that $\varphi$ is a morphism in ${ }_{H}^{H} \mathcal{Y D}$. First note that, for every $r \in R_{n}$, we have

$$
\begin{aligned}
\varphi\left(r+R_{n-1}\right) & =\delta_{|r|, n} \varphi\left(r+R_{n-1}\right)=\delta_{|r|, n} \varphi_{n}\left(r+R_{n-1}\right)=\delta_{|r|, n} \varphi_{n}(\bar{r}) \\
& =\delta_{|r|, n} \overline{r \otimes 1_{H}}=\delta_{|r|, n}\left(r \otimes 1_{H}+\left(R \#_{\xi} H\right)_{n-1}\right)=r \otimes 1_{H}+(R \# \xi H)_{n-1} .
\end{aligned}
$$

Thus

$$
\varphi\left(r+R_{n-1}\right)=r \otimes 1_{H}+\left(R \#_{\xi} H\right)_{n-1}, \text { for every } r \in R_{n} .
$$

For every $r \in R_{n} \backslash R_{n-1}$, by using (32), it is straighforward to prove that $h \triangleright \varphi(\bar{r})=\varphi(h \bar{r})$.

Moreover, by applying (1), (30), the definition of $\pi_{G}$ and (32), we get that $\rho \varphi(\bar{r})=(H \otimes \varphi) \rho(\bar{r})$.

Let us check that $\varphi$ is a morphism of bialgebras in ${ }_{H}^{H} \mathcal{Y D}$. Fix $r \in R_{n} \backslash R_{n-1}$.

Using the definition of $\Delta_{\mathcal{D}}$, (1), (30), the definition of $\pi_{G}$, the definition of $\sigma_{G}$, (32) and (1) again, we obtain $\Delta_{\mathcal{D}} \varphi(\bar{r})=(\varphi \otimes \varphi) \Delta_{\operatorname{gr}(R)}(\bar{r})$.

Let us check $\varphi$ is counitary:

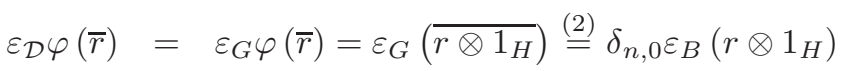

$$
\begin{aligned}
& =\delta_{n, 0} \varepsilon_{R}(r) \stackrel{(\underline{2})}{=} \varepsilon_{\operatorname{gr}(R)}(\bar{r}) \text {. }
\end{aligned}
$$

Let us check $\varphi$ is multiplicative. Let $s \in R_{m} \backslash R_{m-1}$. Then, by definition of $\varphi$, of $m_{\mathcal{D}}$ and of the multiplication of $R \#_{\xi} H$, we have that

$$
m_{\mathcal{D}}(\varphi \otimes \varphi)(\bar{s} \otimes \bar{r})=\sum\left(s^{(1)}\left(\left(s^{(2)}\right)_{\langle-1\rangle} r^{(1)}\right) \# \xi\left(\left(s^{(2)}\right)_{\langle 0\rangle} \otimes r^{(2)}\right)\right)+(R \# \xi H)_{m+n-1} .
$$

Now write $\sum s^{(1)} \otimes s^{(2)}=\sum_{0 \leq i \leq m} s_{i} \otimes s_{m-i}^{\prime}$ for some $s_{i}, s_{i}^{\prime} \in R_{i}$ and similarly $\sum r^{(1)} \otimes r^{(2)}=$ $\sum_{0 \leq j \leq n} r_{j} \otimes r_{n-j}^{\prime}$ for some $r_{j}, r_{j}^{\prime} \in R_{j}$. Then

$$
\begin{aligned}
m_{\mathcal{D}}(\varphi \otimes \varphi)(\bar{s} \otimes \bar{r})= & \sum_{\substack{0 \leq i \leq m \\
0 \leq j \leq n}}\left(s_{i}\left(\left(s_{m-i}^{\prime}\right)_{\langle-1\rangle} r_{j}\right) \# \xi\left(\left(s_{m-i}^{\prime}\right)_{\langle 0\rangle} \otimes r_{n-j}^{\prime}\right)\right)+(R \# \xi H)_{m+n-1} \\
= & \sum_{\substack{0 \leq i \leq m \\
0 \leq j \leq n}} \delta_{i, m} \delta_{j, n}\left(s_{i}\left(\left(s_{m-i}^{\prime}\right)_{\langle-1\rangle} r_{j}\right) \# \xi\left(\left(s_{m-i}^{\prime}\right)_{\langle 0\rangle} \otimes r_{n-j}^{\prime}\right)\right)+\left(R \# \#_{\xi} H\right)_{m+n-1} \\
= & \left.\sum_{\substack{R_{0}=\mathbb{k} 1_{R} \\
=}}\left(s_{m}\left(\left(s_{0}^{\prime}\right)_{\langle-1\rangle} r_{n}\right) \# \xi\left(\left(s_{0}^{\prime}\right)_{\langle 0\rangle} \otimes r_{0}^{\prime}\right)\right)+\left(R \beta_{\xi} H\right)_{m+n-1}\left(s_{0}^{\prime}\right)_{\langle-1\rangle} r_{n}\right) \# \varepsilon_{R}\left(\left(s_{0}^{\prime}\right)_{\langle 0\rangle}\right) \varepsilon_{R}\left(r_{0}^{\prime}\right) 1_{H}+(R \# \xi H)_{m+n-1}
\end{aligned}
$$




$$
\begin{aligned}
& =\sum s_{m} \varepsilon_{R}\left(s_{0}^{\prime}\right) r_{n} \varepsilon_{R}\left(r_{0}^{\prime}\right) \# 1_{H}+(R \# \xi H)_{m+n-1} \\
& =\sum_{\substack{0 \leq i \leq m \\
0 \leq j \leq n}} \delta_{i, m} \delta_{j, n}\left(s_{i} \varepsilon_{R}\left(s_{m-i}^{\prime}\right) r_{j} \varepsilon_{R}\left(r_{m-j}^{\prime}\right) \# 1_{H}\right)+(R \# \xi H)_{m+n-1} \\
& =\sum_{\substack{0 \leq i \leq m \\
0 \leq j \leq n}}\left(s_{i} \varepsilon_{R}\left(s_{m-i}^{\prime}\right) r_{j} \varepsilon_{R}\left(r_{m-j}^{\prime}\right) \# 1_{H}\right)+(R \# \xi H)_{m+n-1} \\
& =\sum\left(s^{(1)} \varepsilon_{R}\left(s^{(2)}\right) r^{(1)} \varepsilon_{R}\left(r^{(2)}\right) \# 1_{H}\right)+\left(R \# H_{\xi} H\right)_{m+n-1} \\
& =\left(s r \# 1_{H}\right)+(R \# \xi H)_{m+n-1} \\
& =\varphi\left(\left(s+R_{m-1}\right)\left(r+R_{n-1}\right)\right)=\varphi m_{\operatorname{gr}(R)}(s r \otimes r) .
\end{aligned}
$$

Let us check $\varphi$ is unitary. We have

$$
\varphi\left(1_{\operatorname{gr}(R)}\right)=\varphi\left(1_{R}+R_{-1}\right)=\varphi\left(\overline{1_{R}}\right)=\overline{1_{R} \otimes 1_{H}}=\left(1_{R} \otimes 1_{H}\right)+(R \# \xi H)_{-1}=1_{B}+B_{-1}=1_{G} .
$$

Summing up we have proved that

$$
\operatorname{gr}(Q) \stackrel{Q=R^{v}}{=} \operatorname{gr}\left(R^{v}\right) \stackrel{\text { Lem. }}{\cong} \operatorname{gr}(R) \stackrel{\text { Pro. }}{\cong} \text {. } 5 \text { D }\left(R \#_{\xi} H\right) \stackrel{\text { Pro. 5.4 }}{=} \mathcal{D}(A)
$$

as bialgebras in ${ }_{H}^{H} \mathcal{Y D}$. Therefore $\mathrm{H}_{\mathcal{Y} \mathcal{D}}^{3}(\mathcal{D}(A), \mathbb{k})=0$ (the Hochschild cohomology in ${ }_{H}^{H} \mathcal{Y} \mathcal{D}$ of the algebra $\mathcal{D}(A)$ with values in $\mathbb{k})$ if, and only if, $\mathrm{H}_{\mathcal{Y} \mathcal{D}}^{3}(\operatorname{gr} Q, \mathbb{k})=0$. In this case, by the foregoing, we get that $Q$ is gauge equivalent to a connected bialgebra in ${ }_{H}^{H} \mathcal{Y D}$.

Now let $E$ be a connected bialgebra in ${ }_{H}^{H} \mathcal{Y D}$ and let $\gamma: E \otimes E \rightarrow \mathbb{k}$ be a gauge transformation in ${ }_{H}^{H} \mathcal{Y D}$ such that $Q=E^{\gamma}$. We proved that $A^{\zeta} \cong Q \# H \cong E^{\gamma} \# H$ as coquasi-bialgebras. By Proposition 2.5, we have that $(E \# H)^{\Gamma}=E^{\gamma} \# H$ as an ordinary coquasi-bialgebras. Recall that two coquasi-bialgebras $A$ and $A^{\prime}$ are called gauge equivalent or quasi-isomorphic whenever there is some gauge transformation $\gamma: Q \otimes Q \rightarrow \mathbb{k}$ in $\mathbf{V e c}_{\mathbb{k}}$ such that $A^{\gamma} \cong A^{\prime}$ as coquasi-bialgebras. We point out that, if $A$ and $A^{\prime}$ are ordinary bialgebras and $A^{\gamma} \cong A^{\prime}$, then $\gamma$ comes out to be a unitary cocycle. This is encoded in the triviality of the reassociators of $A$ and $A^{\prime}$.

TheOREM 5.6. Let $A$ be a finite-dimensional Hopf algebra over a field $\mathbb{k}$ of characteristic zero such that the coradical $H$ of $A$ is a sub-Hopf algebra (i.e. A has the dual Chevalley Property). If $\mathrm{H}_{\mathcal{Y} \mathcal{D}}^{3}(\mathcal{D}(A), \mathbb{k})=0$, then $A$ is quasi-isomorphic to the Radford-Majid bosonization E\#H of some connected bialgebra $E$ in ${ }_{H}^{H} \mathcal{Y D}$ by $H$. Moreover $\operatorname{gr}(E) \cong \mathcal{D}(A)$ as bialgebras in ${ }_{H}^{H} \mathcal{Y D}$.

Proof. By the foregoing $A^{\zeta} \cong Q \# H \cong E^{\gamma} \# H=(E \# H)^{\Gamma}$ as coquasi-bialgebras. Now $A$ is quasi-isomorphic to $A^{\zeta}$ which is quasi-isomorphic to $E \# H$ so that $A$ is quasi-isomorphic to $E \# H$. Moreover

$$
\operatorname{gr}(E)=\operatorname{gr}\left(E^{\gamma}\right)=\operatorname{gr}(Q) \cong \mathcal{D}(A) .
$$

where the first equality holds by Proposition 2.6 .

More generally, given $A$ a (finite-dimensional) Hopf algebra whose coradical $H$ is a sub-Hopf algebra, then if $H$ is also semisimple, we expect that $A$ is quasi-isomorphic to the Radford-Majid bosonization $E \# H$ of some connected bialgebra $E$ in ${ }_{H}^{H} \mathcal{Y D}$ by $H$. See e.g. GM, Corollary 3.4 and the proof therein] and [AAGMV, AAG] for a further clue in this direction.

\section{ExAmples}

We notice that the Hochschild cohomology of a finite-dimensional Nichols algebras has been computed in few examples. We consider here those Nichols algebras to compute $\mathrm{H}_{\mathcal{Y} \mathcal{D}}^{3}(\mathcal{B}(V), \mathbb{k})$. 
6.1. Braidings of Cartan type. Let $A=\left(a_{i j}\right)_{1 \leq i, j \leq \theta}$ be a finite Cartan matrix, $\Delta$ the corresponding root system, $\left(\alpha_{i}\right)_{1 \leq i \leq \theta}$ a set of simple roots and $W$ its Weyl group. Let $w_{0}=s_{i_{1}} \cdots s_{i_{M}}$ be a reduced expression of the element $w_{0} \in W$ of maximal length as a product of simple reflections, $\beta_{j}=s_{i_{1}} \cdots s_{i_{j-1}}\left(\alpha_{i_{j}}\right), 1 \leq j \leq M$. Then $\beta_{j} \neq \beta_{k}$ if $j \neq k$ and $\Delta^{+}=\left\{\beta_{j} \mid 1 \leq j \leq M\right\}$, see [H], page 25 and Proposition 3.6].

Let $\Gamma$ be a finite abelian group, $\widehat{\Gamma}$ its group of characters. $\mathcal{D}=\left(\Gamma,\left(g_{i}\right)_{1 \leq i \leq \theta},\left(\chi_{i}\right)_{1 \leq i \leq \theta}, A\right)$ is a datum of finite Cartan type AS2 associated to $\Gamma$ and $A$ if $g_{i} \in \Gamma, \chi_{j} \in \widehat{\Gamma}, 1 \leq i, j \leq \theta$, satisfy $\chi_{i}\left(g_{i}\right) \neq 1, \chi_{i}\left(g_{j}\right) \chi_{j}\left(g_{i}\right)=\chi_{i}\left(g_{i}\right)^{a_{i j}}$ for all $i, j$. Set $\mathfrak{q}=\left(q_{i j}\right)_{1 \leq i, j \leq \theta}$, where $q_{i j}=\chi_{j}\left(g_{i}\right)$.

In what follows $V$ denotes the Yetter-Drinfeld module over $\mathbb{k} \Gamma$, $\operatorname{dim} V=\theta$, with a fixed basis $x_{1}, \ldots, x_{\theta}$, where the action and the coaction over each $x_{i}$ is given by $\chi_{i}$ and $g_{i}$, respectively. Then the associated braiding is $c\left(x_{i} \otimes x_{j}\right)=q_{i j} x_{j} \otimes x_{i}$ for all $i, j$. Let $\mathcal{B}_{\mathfrak{q}}=\mathcal{B}(V)$. The tensor algebra $T(V)$ is $\mathbb{N}_{0}^{\theta}$-graded with grading $\alpha_{i}$ for each $x_{i}$. For $\beta=\sum_{i=1}^{\theta} a_{i} \alpha_{i} \in \Delta^{+}$, set

$$
g_{\beta}=g_{1}^{a_{1}} \cdots g_{\theta}^{a_{\theta}}, \quad \chi_{\beta}=\chi_{1}^{a_{1}} \cdots \chi_{\theta}^{a_{\theta},} \quad q_{\beta}=\chi_{\beta}\left(g_{\beta}\right) .
$$

Given $\alpha, \beta \in \Delta^{+}$, we denote $q_{\alpha \beta}=\chi_{\beta}\left(g_{\alpha}\right)$.

We assume as in AS2, MPSW that the order of $q_{i i}$ is odd for all $i$, and not divisible by 3 for each connected component of the Dynkin diagram of $A$ of type $G_{2}$. Therefore the order of $q_{i i}$ is the same for all the $i$ in the same connected component $J$. Given $\beta \in J$, we denote by $N_{\beta}$ the order of the corresponding $q_{i i}$ in $J$, which is also the order of $q_{\beta}$.

By [I] there exist homogeneous elements $x_{\beta}$ of degree $\beta, \beta \in \Delta^{+}$, such that the Nichols algebra $\mathcal{B}_{\mathfrak{q}}$ of $V$ is presented by generators $x_{1}, \ldots, x_{\theta}$ and relations

$$
\begin{aligned}
\left(\operatorname{ad}_{c} x_{i}\right)^{1-a_{i j}} x_{j} & =0, & & 1 \leq i \neq j \leq \theta ; \\
x_{\beta}^{N_{\beta}} & =0, & & \beta \in \Delta_{+} .
\end{aligned}
$$

Moreover $\left\{x_{\beta_{1}}^{n_{1}} \ldots x_{\beta_{M}}^{n_{M}} \mid 0 \leq n_{i}<N_{\beta_{i}}\right\}$ is a basis of $\mathcal{B}_{\mathfrak{q}}$.

We shall prove that $\mathrm{H}_{\mathcal{Y} \mathcal{D}}^{3}\left(\mathcal{B}_{\mathfrak{q}}, \mathbb{k}\right)=0$. We need first some technical results.

Lemma 6.1. Let $\alpha, \beta \in \Delta_{+}$. Then either $g_{\alpha} g_{\beta}^{N_{\beta}} \neq e$, or else $\chi_{\alpha} \chi_{\beta}^{N_{\beta}} \neq \epsilon$.

Proof. Suppose on the contrary that $g_{\alpha} g_{\beta}^{N_{\beta}}=e, \chi_{\alpha} \chi_{\beta}^{N_{\beta}}=\epsilon$. Then

$$
q_{\alpha}=\chi_{\alpha}^{-1}\left(g_{\alpha}^{-1}\right)=\chi_{\beta}^{N_{\beta}}\left(g_{\beta}^{N_{\beta}}\right)=q_{\beta}^{N_{\beta}^{2}}=1,
$$

since $q_{\beta}$ is a root of unity of order $N_{\beta}$. But this is a contradiction, since $q_{\alpha} \neq 1$.

Lemma 6.2. Let $\alpha, \beta, \gamma \in \Delta^{+}$be pairwise different. Then either $g_{\alpha} g_{\beta} g_{\gamma} \neq e$, or else $\chi_{\alpha} \chi_{\beta} \chi_{\gamma} \neq \epsilon$.

Proof. Suppose on the contrary that $g_{\alpha} g_{\beta} g_{\gamma}=e$ and $\chi_{\alpha} \chi_{\beta} \chi_{\gamma}=\epsilon$. Then

$$
q_{\alpha}=\chi_{\alpha}^{-1}\left(g_{\alpha}^{-1}\right)=\chi_{\beta} \chi_{\gamma}\left(g_{\beta} g_{\gamma}\right)=q_{\beta} q_{\gamma} q_{\beta \gamma} q_{\gamma \beta}, \quad q_{\beta}=q_{\alpha} q_{\gamma} q_{\alpha \gamma} q_{\gamma \alpha}, \quad q_{\gamma}=q_{\alpha} q_{\beta} q_{\alpha \beta} q_{\beta \alpha} .
$$

Notice that $\alpha, \beta, \gamma$ belong to the same connected component. Indeed, if $\gamma$ belongs to a different connected component, then $q_{\beta \gamma} q_{\gamma \beta}=q_{\alpha \gamma} q_{\gamma \alpha}=1$. Thus $q_{\beta}=q_{\alpha} q_{\gamma}=q_{\beta} q_{\gamma}^{2}$, so $q_{\gamma}^{2}=1$, which is a contradiction. Therefore we may assume that the Dynkin diagram is connected.

One can prove that $q_{s_{i}(\alpha)}=q_{\alpha}$ for every $\alpha \in \Delta$. As we observed that $\Delta^{+}=\left\{\beta_{j} \mid 1 \leq j \leq M\right\}$, we deduce that for every $\beta \in \Delta^{+}$there is some $j$ such that $q_{\beta}=q_{j}$. One can prove that there is some $q \in \mathbb{k}$ such that $q_{\alpha}=q^{(\alpha, \alpha) / 2}$ and $q_{\alpha \gamma} q_{\gamma \alpha}=q^{(\alpha, \gamma)}$, where $(\cdot, \cdot)$ is the invariant bilinear form on the simple Lie algebra $\mathfrak{g}$ associated with the finite Cartan matrix $\mathrm{B}$, Ch. VI, $\S 1$, Proposition 3 and Definition 3] and the basis of the root systems given in [BO, Ch. VI, §4] should be normalized in such a way that $q=q_{\delta},(\delta, \delta)=2$ for each short root $\delta \in \Delta$. Note that $q_{\alpha}=q^{(\alpha, \alpha) / 2} \neq 1$ for all $\alpha$ as $(\alpha, \alpha) \neq 0$. Thus

- $q_{\alpha}=q_{\beta}=q_{\gamma}=q$ if the Dynkin diagram is simply laced,

- $q_{\alpha}, q_{\beta}, q_{\gamma} \in\left\{q, q^{2}\right\}$ if the Dynkin diagram has a double arrow,

- $q_{\alpha}, q_{\beta}, q_{\gamma} \in\left\{q, q^{3}\right\}$ if the Dynkin diagram is of type $G_{2}$. 
If the Dynkin diagram is simply laced, then, by (33), we have $q_{\beta \gamma} q_{\gamma \beta}=q_{\alpha \gamma} q_{\gamma \alpha}=q_{\alpha \beta} q_{\beta \alpha}=q^{-1}$. Then $q^{(\alpha, \gamma)}=q^{-1}$. Now set $n(\alpha, \beta):=2(\alpha, \beta) /(\beta, \beta)=(\alpha, \beta)$. Then $n(\alpha, \beta)$ is symmetric whence, by [Bo, Ch. VI, $\S 1$, page 148] we have $(\alpha, \gamma)=-1$ as the order of $q$ is odd, so $\alpha+\gamma \in \Delta^{+}$, by [B], VI, $\S 1$, Corollary, page 149]. Now the same argument we used above shows that also $(\alpha, \beta)=-1=(\gamma, \beta)$ and hence $(\alpha+\gamma, \beta)=-2$, so $\alpha+\beta+\gamma \in \Delta^{+}$, since $\alpha+\gamma \neq-\beta$ (as $\alpha+\gamma$ and $\beta$ are both in $\Delta^{+}$). But $q_{\alpha+\beta+\gamma}=q_{\alpha} q_{\beta} q_{\gamma} q_{\beta \gamma} q_{\gamma \beta} q_{\alpha \gamma} q_{\gamma \alpha} q_{\alpha \beta} q_{\beta \alpha}=1$, which is a contradiction.

If the Dynkin diagram has a double arrow, then $q_{\alpha}, q_{\beta}, q_{\gamma} \in\left\{q, q^{2}\right\}$. If $q_{\alpha}=q_{\beta}=q_{\gamma}$, then the proof follows as for the simply-laced case because $n(u, v)=n(v, u)$ for $u, v \in\{\alpha, \beta, \gamma\}$. If $q_{\alpha}=q_{\beta}=q$ and $q_{\gamma}=q^{2}$, then $q_{\beta \gamma} q_{\gamma \beta}=q_{\alpha \gamma} q_{\gamma \alpha}=q^{-2}$, and $q_{\alpha \beta} q_{\beta \alpha}=1$, by (33). Then a simple calculation yields $(\beta, \gamma)=-2$ so that $\beta+\gamma \in \Delta^{+}$. One also gets $(\alpha, \beta)=0$ and $(\alpha, \gamma)=-2$ so that $(\alpha, \beta+\gamma)=(\alpha, \beta)+(\alpha, \gamma)=-2<0$ by the conditions on the order of $q$, so again $\alpha+\beta+\gamma \in \Delta^{+}$; but again we obtain $q_{\alpha+\beta+\gamma}=1$, which is a contradiction. The proof for $q_{\alpha}=q_{\beta}=q^{2}$ and $q_{\gamma}=q$ follows analogously.

Finally, if the Dynkin diagram is of type $G_{2}$, then a similar analysis gives a contradiction.

For each $1 \leq k \leq M$, set $\mathcal{B}_{\mathfrak{q}}(k)$ as the subspace of $\mathcal{B}_{\mathfrak{q}}$ spanned by $\left\{x_{\beta_{1}}^{n_{1}} \ldots x_{\beta_{k}}^{n_{k}} \mid 0 \leq n_{i}<N_{\beta_{i}}\right\}$. By DP this gives an algebra filtration, and the graded algebra $\operatorname{Gr} \mathcal{B}_{\mathfrak{q}}$ associated to this filtration is presented by generators $\mathbf{x}_{\beta}, \beta \in \Delta^{+}$, and relations

$$
\mathbf{x}_{\beta} \mathbf{x}_{\gamma}=q_{\beta \gamma} \mathbf{x}_{\gamma} \mathbf{x}_{\beta}, \quad \quad \mathbf{x}_{\beta}^{N_{\beta}}=0, \quad \beta<\gamma \in \Delta_{+} .
$$

In MPSW] $\operatorname{Gr} \mathcal{B}_{\mathfrak{q}}$ is viewed as an algebra in $\underset{\mathbb{k} \Gamma}{\mathbb{k} \Gamma} \mathcal{Y} \mathcal{D}$, which (as an algebra) is the Nichols algebra of Cartan type $A_{1} \times \cdots \times A_{1}, M$ copies, with action and coaction on $\mathbf{x}_{\beta}$ given by $\chi_{\beta}, g_{\beta}$, respectively. By [MPSW, Theorem 4.1$], \mathrm{H}^{\bullet}\left(\operatorname{Gr} \mathcal{B}_{\mathfrak{q}}, \mathbb{k}\right)$ is the algebra generated by $\xi_{\beta}, \eta_{\beta}, \beta \in \Delta^{+}$, where $\operatorname{deg} \xi_{\beta}=2, \operatorname{deg} \eta_{\beta}=1$, and relations

$$
\xi_{\beta} \xi_{\gamma}=q_{\beta \gamma}^{N_{\beta} N_{\gamma}} \xi_{\gamma} \xi_{\beta}, \quad \eta_{\beta} \xi_{\gamma}=q_{\beta \gamma}^{N_{\gamma}} \xi_{\gamma} \eta_{\beta}, \quad \eta_{\beta} \eta_{\gamma}=-q_{\beta \gamma} \eta_{\gamma} \eta_{\beta}, \quad \beta, \gamma \in \Delta^{+} .
$$

As we assume that all the $q_{i i}$ have odd order, we deduce in particular from the last equality that $\eta_{\beta}^{2}=0$ for all $\beta \in \Delta^{+}$. As an algebra in ${ }_{\mathbb{k} \Gamma}^{\mathbb{k} \Gamma} \mathcal{Y} \mathcal{D}$, the action and coaction on $\xi_{\beta}$ is given by $\chi_{\beta}^{-N_{\beta}}$, $g_{\beta}^{-N_{\beta}}$, while the action and coaction on $\eta_{\beta}$ is given by $\chi_{\beta}^{-1}, g_{\beta}^{-1}$.

THEOREM 6.3. $\mathrm{H}_{\mathcal{Y} \mathcal{D}}^{3}\left(\mathcal{B}_{\mathfrak{q}}, \mathbb{k}\right)=0$.

Proof. First we will prove that $\mathrm{H}^{3}\left(\operatorname{Gr} \mathcal{B}_{\mathfrak{q}}, \mathbb{k}\right)^{D}=0$ for $D:=D(\mathbb{k} \Gamma)$. Now, the invariants are with respect to the $D$-bimodule structure that $\mathrm{H}^{3}\left(\operatorname{Gr} \mathcal{B}_{\mathfrak{q}}, \mathbb{k}\right)$ inherits from $\operatorname{Hom}\left(\left(\operatorname{Gr} \mathcal{B}_{\mathfrak{q}}\right)^{\otimes 3}, \mathbb{k}\right)$ (this is a $D$-bimodule as its arguments are left $D$-modules). Since the left $D$-module structure is induced by the one of $\mathbb{k}$, it is trivial. Thus the invariants of $\mathrm{H}^{3}\left(\operatorname{Gr} \mathcal{B}_{\mathfrak{q}}, \mathbb{k}\right)$ as a $D$-bimodule reduce to the its invariants as a right $D$-module. Since right $D$-modules are equivalent to left $D$-modules, via the antipode of $D$ which is invertible as $D$ is finite-dimensional, the right $D$-module structure of $\mathrm{H}^{3}\left(\operatorname{Gr} \mathcal{B}_{\mathfrak{q}}, \mathbb{k}\right)$ becomes the structure of object in ${ }_{\mathbb{k} \Gamma}^{\mathrm{k}} \Gamma \mathcal{Y} \mathcal{D}$ described above. Thus, in order to prove that $\mathrm{H}^{3}\left(\operatorname{Gr} \mathcal{B}_{\mathfrak{q}}, \mathbb{k}\right)^{D}=0$ we just have to check that the invariants of $\mathrm{H}^{3}\left(\mathrm{Gr} \mathcal{B}_{\mathfrak{q}}, \mathbb{k}\right)$ as a left-left Yetter-Drinfeld modules are zero.

Now, by the defining relations of $\mathrm{H}^{\bullet}\left(\mathrm{Gr} \mathcal{B}_{\mathfrak{q}}, \mathbb{k}\right)$, a basis $B$ of $\mathrm{H}^{3}\left(\operatorname{Gr} \mathcal{B}_{\mathfrak{q}}, \mathbb{k}\right)$ is given by $\left\{\xi_{\alpha} \eta_{\beta}\right\} \cup$ $\left\{\eta_{\alpha} \eta_{\beta} \eta_{\gamma} \mid \alpha<\beta<\gamma\right\}$. If $v \in \mathrm{H}^{3}\left(\operatorname{Gr} \mathcal{B}_{\mathfrak{q}}, \mathbb{k}\right)$ is invariant, then $v$ is written as a linear combination of elements in the trivial component. Indeed, write $v=\sum_{b \in B} c_{b} b$ for some $c_{b} \in \mathbb{k}$, and let $g_{b}, \chi_{b}$ be the elements describing the component of $b \in B$. Then

$$
\begin{aligned}
& v=g \cdot v=\sum_{b \in B} c_{b} g \cdot b=\sum_{b \in B} c_{b} \chi_{b}(g) b, \quad \text { for all } g \in \Gamma, \\
& 1 \otimes v=\rho(v)=\sum_{b \in B} c_{b} \rho \cdot b=\sum_{b \in B} c_{b} g_{b} \otimes b .
\end{aligned}
$$

If $c_{b} \neq 0$, then $\chi_{b}(g)=1$ for all $g \in \Gamma$ so $\chi_{b}=\epsilon$, and $g_{b}=1$. Thus $b$ is invariant. We have so proved that the existence of $v \neq 0$ invariant implies the existence of $b \in B$ invariant. Hence, if $B$ has no invariant element then there is no invariant element at all. Note that, for all $h \in H$, we have $h \cdot\left(\xi_{\alpha} \eta_{\beta}\right)=\left(\chi_{\alpha}^{-N_{\alpha}} \chi_{\beta}^{-1}\right)(h) \xi_{\alpha} \eta_{\beta}$ and $\rho\left(\xi_{\alpha} \eta_{\beta}\right)=g_{\alpha}^{-N_{\alpha}} g_{\beta}^{-1} \otimes \xi_{\alpha} \eta_{\beta}$ so that, by Lemma 6.1, the element 
$\xi_{\alpha} \eta_{\beta}$ is not $D$-invariant. A similar argument, using Lemma 6.2 shows that also $\eta_{\alpha} \eta_{\beta} \eta_{\gamma}$ is not $D$ invariant. Thus the elements in $B$ are not $D$-invariant, so $\mathrm{H}^{3}\left(\operatorname{Gr} \mathcal{B}_{\mathfrak{q}}, \mathbb{k}\right)^{D}=0$. Since the elements in $\left\{x_{\beta_{1}}^{n_{1}} \ldots x_{\beta_{k}}^{n_{k}} \mid 0 \leq n_{i}<N_{\beta_{i}}\right\}$ are eigenvectors for $D$, we can mimic the argument in MPSW, Section $5]$ by taking into account the spectral sequence associated to the filtration of algebras therein; see for example MPSW, Corollary 5.5] for a similar argument. Thus $\mathrm{H}_{\mathcal{Y} \mathcal{D}}^{3}\left(\mathcal{B}_{\mathfrak{q}}, \mathbb{k}\right) \cong \mathrm{H}^{3}\left(\mathcal{B}_{\mathfrak{q}}, \mathbb{k}\right)^{D}=0$.

REMARK 6.4. Notice that $\mathrm{H}_{\mathcal{Y} \mathcal{D}}^{3}\left(\mathcal{B}_{\mathfrak{q}}, \mathbb{k}\right) \cong \mathrm{H}^{3}\left(\mathcal{B}_{\mathfrak{q}}, \mathbb{k}\right)^{D(\mathbb{k} \Gamma)}=0$ although $\mathrm{H}^{3}\left(\mathcal{B}_{\mathfrak{q}} \# \mathbb{k} \Gamma, \mathbb{k}\right) \cong \mathrm{H}^{3}\left(\mathcal{B}_{\mathfrak{q}}, \mathbb{k}\right)^{\Gamma}$ can be non-trivial, see for example [MPSW, Example 5.8].

6.2. Braidings of non-diagonal type. For $n \geq 3, \mathcal{F} \mathcal{K}_{n}$ denotes the quadratic algebra [FK] with a presentation by generators $x_{(i j)}, 1 \leq i<j \leq n$, and relations

$$
\begin{aligned}
x_{(i j)}^{2} & =0, & & 1 \leq i<j \leq n, \\
x_{(i j)} x_{(j k)} & =x_{(j k)} x_{(i k)}+x_{(i k)} x_{(i j)}, & & 1 \leq i<j<k \leq n, \\
x_{(j k)} x_{(i j)} & =x_{(i k)} x_{(j k)}+x_{(i j)} x_{(i k)}, & & 1 \leq i<j<k \leq n, \\
x_{(i j)} x_{(k l)} & =x_{(k l)} x_{(i j)}, & & \#\{i, j, k, l\}=4 .
\end{aligned}
$$

According to MiS each $\mathcal{F} \mathcal{K}_{n}$ is a graded bialgebra in the category of Yetter-Drinfeld modules over the symmetric group $S_{n}$, generated as an algebra by the vector space $V_{n}$ with basis $\left\{x_{(i j)} \mid 1 \leq\right.$ $i<j \leq n\}$. The action is described by identifying $(i j)$ with the corresponding transposition in $S_{n}$ and then consider the conjugation twisted by the sign, while the coaction is given by declaring $x_{\sigma}$ a homogeneous element of degree $\sigma$. Then the braiding on $V_{n}$ becomes

$$
c\left(x_{\sigma} \otimes x_{\tau}\right)=\chi(\sigma, \tau) x_{\sigma \tau \sigma^{-1}} \otimes x_{\sigma}, \quad \chi(\sigma, \tau)= \begin{cases}1 & \sigma(i)<\sigma(j), \tau=(i j), i<j, \\ -1 & \text { otherwise },\end{cases}
$$

where $\sigma$ and $\tau$ are transpositions. Moreover $\mathcal{F K}_{n}$ projects onto the Nichols algebra $\mathcal{B}\left(V_{n}\right)$. For $n=3,4,5$, it is known that $\mathcal{F} \mathcal{K}_{n}=\mathcal{B}\left(V_{n}\right)$ and has dimension, respectively, 12,576 and 8294400 .

The Hochschild cohomology of $\mathcal{F} \mathcal{K}_{3}$ is a consequence of the results in $\mathrm{SV}$ ] as follows.

TheOREM 6.5. $\mathrm{H}_{\mathbb{k} S_{3}-\mathrm{Mod}}^{\bullet}\left(\mathcal{F} \mathcal{K}_{3}, \mathbb{k}\right)$ is isomorphic to the graded algebra

$$
\mathbb{k}[X, U, V] /\left(U^{2} V-V U^{2}\right), \quad \text { where } \operatorname{deg} U=\operatorname{deg} V=2, \operatorname{deg} X=4 .
$$

Proof. By [SV, Theorem 4.19], we have that $E\left(B \# \mathbb{k} S_{3}\right)$ is isomorphic to the algebra in the claim, where $B=\mathcal{F} \mathcal{K}_{3}$. By [SV], Theorem 2.17], we know that $E\left(B \# \mathbb{k} S_{3}\right) \cong E(B)^{\mathbb{k} S_{3}}$ as graded algebras. As observed in Remark 1.2, we have that $E(B) \cong \mathrm{H}^{\bullet}(B, \mathbb{k})$. By Remark 4.6 , we have $\mathrm{H}^{\bullet}(B, \mathbb{k})^{\mathbb{k} S_{3}} \cong$ $\mathrm{H}_{\mathrm{k}, S_{3}-\operatorname{Mod}}^{\bullet}\left(\mathcal{F} \mathcal{K}_{3}, \mathbb{k}\right)$.

From this result we get $\mathrm{H}_{\mathrm{k} S_{3}-\mathrm{Mod}}^{3}\left(\mathcal{F} \mathcal{K}_{3}, \mathbb{k}\right)=0$ so that, by Proposition 4.7 we conclude that

Corollary 6.6. $\mathrm{H}_{\mathcal{Y} \mathcal{D}}^{3}\left(\mathcal{F} \mathcal{K}_{3}, \mathbb{k}\right)=0$.

\section{REFERENCES}

[ABM1] A. Ardizzoni, M. Beattie and C. Menini, Cocycle Deformations for Hopf Algebras with a Coalgebra Projection, J. Algebra, Vol. 324(4) (2010) 673-705.

$[\mathrm{ABM}]$ A. Ardizzoni, M. Beattie and C. Menini, Gauge Deformations for Hopf Algebras with the Dual Chevalley Property, J. Algebra Appl., Vol. 11(3) (2012) 1250051.

[AAG] N. Andruskiewitsch. I. E. Angiono, A. García Iglesias, Liftings of Nichols algebras of diagonal type I. Cartan type A. (arXiv:1509.01622)

[AAGMV] N. Andruskiewitsch, I. E. Angiono, A. García Iglesias, A. Masuoka and C. Vay, Liftings via cocycle deformations. J. Pure Appl. Alg. 218 (2014), 684-703.

[AM] A. Ardizzoni and C. Menini, Associated Graded Algebras and Coalgebras, Comm. Algebra, Vol. 30(3) (2012), 862-896.

[AMS1] A. Ardizzoni, C. Menini and D. Ştefan, A Monoidal Approach to Splitting Morphisms of Bialgebras, Trans. Amer. Math. Soc., 359 (2007), 991-1044.

[AMS2] A. Ardizzoni, C. Menini and D. Ştefan, Hochschild Cohomology And 'Smoothness' In Monoidal Categories, J. Pure Appl. Algebra, Vol. 208 (2007), 297-330. 
[AMStu] A. Ardizzoni, C. Menini and F. Stumbo, Small Bialgebras with a Projection, J. Algebra Vol. 314(2) (2007), 613-663.

[An] I. E. Angiono, Basic quasi-Hopf algebras over cyclic groups. Adv. Math. 225 (2010), no. 6, 3545-3575.

[AP] A. Ardizzoni, A. Pavarin, Bosonization for Dual Quasi-Bialgebras and Preantipode, J. Algebra, Vol. 390 (2013), Pages 126-159.

[AS1] N. Andruskiewitsch, H.-J. Schneider, Lifting of quantum linear spaces and pointed Hopf algebras of order $p^{3}$. J. Algebra 209 (1998), no. 2, 658-691.

[AS2] N. Andruskiewitsch, H-J. Schneider, On the classification of finite-dimensional pointed Hopf algebras, Ann. of Math. 171 (2010), no. 1, 375-417.

[Bo] N. Bourbaki, Groupes et algèbres de Lie, Chapitres 4, 5 et 6, Hermann, Paris, 1968.

[Br] K. S. Brown, Cohomology of groups. Graduate Texts in Mathematics, 87. Springer-Verlag, New YorkBerlin, 1982.

[CE] H. Cartan, S. Eilenberg, Homological algebra. Princeton University Press, Princeton, N. J., 1956.

[DNR] S. Dăscălescu, C. Năstăsescu, S. Raianu, Hopf algebras. An introduction. Monographs and Textbooks in Pure and Applied Mathematics, 235. Marcel Dekker, Inc., New York, 2001.

[DP] C. De Concini, C. Procesi, Quantum groups. D-modules, representation theory, and quantum groups, 31140, Lecture Notes in Math. 1565, Springer, 1993.

[EG] P. Etingof, S. Gelaki, Liftings of graded quasi-Hopf algebras with radical of prime codimension. J. Pure Appl. Algebra 205 (2006), no. 2, 310-322.

[FK] S. Fomin, A. N. Kirillov, Quadratic algebras, Dunkl elements and Schubert calculus, Progr. Math. 172 (1999), 146-182.

[GiK] V. Ginzburg, S. Kumar, Cohomology of quantum groups at roots of unity. Duke Math. J. 69 (1993) 179-198.

[GM] L. Grunenfelder; M. Mastnak, Pointed Hopf Algebras As Cocycle Deformations, preprint. (arXiv:1010. 4976v1

[H] H. Hiller, Geometry of Coxeter groups. Research Notes in Mathematics, 54. Pitman (Advanced Publishing Program), Boston, Mass.-London, 1982.

[L] G. Lusztig, Quantum groups at roots of 1. Geom. Dedicata 35 (1990), 89-113.

[K] C. Kassel, Quantum groups. Graduate Texts in Mathematics, 155. Springer-Verlag, New York, 1995.

[Maj] S. Majid, Foundations of quantum group theory. Cambridge University Press, Cambridge, 1995.

[MPSW] M. Mastnak, J. Pevtsova, P. Schauenburg, S. Witherspoon, Cohomology of finite-dimensional pointed Hopf algebras. Proc. Lond. Math. Soc. 100 (2010), 377-404.

[MiS] A. Milinski, H.J. Schneider, Pointed indecomposable Hopf algebras over Coxeter groups, Contemp. Math. 267 (2000), 215-236.

[Mo] S. Montgomery, Hopf Algebras and their actions on rings, CMBS Regional Conference Series in Mathematics 82, 1993.

[Ra1] D. E. Radford, Hopf algebras. Series on Knots and Everything, 49. World Scientific Publishing Co. Pte. Ltd., Hackensack, NJ, 2012.

[Ra2] D. E. Radford, Minimal quasitriangular Hopf algebras, J. Algebra 157 (1993), 285-315.

[Ra3] D. E. Radford, The structure of Hopf algebras with a projection. J. Algebra 92 (1985), no. 2, 322-347.

[RT] D. E. Radford, J. Towber, Yetter-Drinfel'd categories associated to an arbitrary bialgebra. J. Pure Appl. Algebra 87 (1993), no. 3, 259-279.

[Sc] P. Schauenburg, Faithful flatness over Hopf subalgebras: counterexamples. Interactions between ring theory and representations of algebras (Murcia), 331-344, Lecture Notes in Pure and Appl. Math., 210, Dekker, New York, 2000.

[Stf] D. Ştefan, Hochschild cohomology on Hopf Galois extensions. J. Pure Appl. Algebra 103 (1995), no. 2, 221-233.

[Stn] B. Stenström, Rings of quotients Die Grundlehren der Mathematischen Wissenschaften, Band 21\%. An introduction to methods of ring theory. Springer-Verlag, New York-Heidelberg, 1975.

[SV] D. Sttefan, C. Vay, The cohomology ring of the 12-dimensional Fomin-Kirillov algebra. arXiv:1404.5101.

[Sw] M. E. Sweedler, Hopf algebras. Mathematics Lecture Note Series W. A. Benjamin, Inc., New York 1969.

[W] X. Wang, Semisimple connected Hopf Algebras. (arXiv:1212.0622v1) 
Famaf-Ciem (COnicet), Universidad Nacional de Córdoba, Medina Allende s/n, Ciudad Universitaria, 5000 Córdoba, Argentina.

E-mail address: angiono@famaf.unc.edu.ar

$U R L$ : www.mate.uncor.edu/ angiong

University of Turin, Department of Mathematics "G. Peano", via Carlo Alberto 10, I-10123 Torino, ITALY

E-mail address: alessandro.ardizzoni@unito.it

$U R L:$ sites.google.com/site/aleardizzonihome

University of Ferrara, Department of Mathematics and Computer Science, Via Machiavelli 35, Ferrara, I-44121, ITALY

E-mail address: men@unife.it

$U R L:$ sites.google.com/a/unife.it/claudia-menini 